\title{
COSMOLOGY WITH PHOTOMETRICALLY CLASSIFIED TYPE Ia SUPERNOVAE FROM THE SDSS-II SUPERNOVA SURVEY
}

\author{
Heather Campbell ${ }^{1}$, Chris B D’Andrea ${ }^{1}$, Robert C. Nichol ${ }^{1}$, Masao Sako $^{2}$, Mathew Smith $^{1,3}$, Hubert Lampeitl $^{1}$, \\ Matthew D. Olmstead ${ }^{4}$, Bruce Bassett ${ }^{5,6,7}$, Rahul Biswas ${ }^{8}$, Peter Brown ${ }^{4,9}$, David Cinabro ${ }^{10}$, Kyle S. Dawson ${ }^{4}$, \\ Ben Dilday ${ }^{11,12}$, Ryan J. Foley ${ }^{13,30}$, Joshua A. Frieman ${ }^{14,15,16}$, Peter Garnavich $^{17}$, Renee HlozeK ${ }^{18}$, Saurabh W. Jha ${ }^{19}$, \\ Steve KuhlmanN $^{8}$, Martin Kunz ${ }^{7,20}$, John Marriner ${ }^{16}$, Ramon Miquel ${ }^{21,22}$, Michael Richmond ${ }^{23}$, Adam Riess ${ }^{24,25}$, \\ Donald P. Schneider ${ }^{26,27}$, JesPer Sollerman ${ }^{28}$, MAtT TAYlor $^{10}$, And Gong-Bo ZhaO ${ }^{1,29}$ \\ ${ }^{1}$ Institute of Cosmology and Gravitation, University of Portsmouth, Portsmouth, PO1 3FX, UK; Heather.Campbell@ port.ac.uk \\ ${ }^{2}$ Department of Physics and Astronomy, University of Pennsylvania, 209 South 33rd Street, Philadelphia, PA 19104, USA \\ ${ }^{3}$ Department of Physics, University of Western Cape, Bellville 7530, Cape Town, South Africa \\ ${ }^{4}$ Department of Physics \& Astronomy, University of Utah, 115 South 1400 East 201, Salt Lake City, UT 84112, USA \\ ${ }^{5}$ Mathematics Department, University of Cape Town, Rondebosch, Cape Town, South Africa \\ ${ }^{6}$ South African Astronomical Observatory, Observatory, Cape Town, South Africa \\ ${ }^{7}$ African Institute for Mathematical Sciences, Muizenberg, 7945, Cape Town, South Africa \\ ${ }^{8}$ Argonne National Laboratory, 9700 South Cass Avenue, Lemont, IL 60439, USA \\ ${ }^{9}$ George P. and Cynthia Woods Mitchell Institute for Fundamental Physics \& Astronomy, Department of Physics and Astronomy, \\ Texas A. \& M. University, 4242 TAMU, College Station, TX 77843, USA \\ ${ }^{10}$ Department of Physics and Astronomy, Wayne State University, Detroit, MI 48126, USA \\ ${ }^{11}$ Las Cumbres Observatory Global Telescope Network, 6740 Cortona Dr., Suite 102, Goleta, CA 93117, USA \\ 12 Department of Physics, University of California, Santa Barbara, Broida Hall, Santa Barbara, CA 93106-9530, USA \\ ${ }^{13}$ Harvard-Smithsonian Center for Astrophysics, 60 Garden Street, Cambridge, MA 02138, USA \\ ${ }^{14}$ Department of Astronomy and Astrophysics, The University of Chicago, 5640 South Ellis Avenue, Chicago, IL 60637, USA \\ ${ }^{15}$ Kavli Institute for Cosmological Physics, The University of Chicago, 5640 South Ellis Avenue Chicago, IL 60637, USA \\ ${ }^{16}$ Center for Particle Astrophysics, Fermi National Accelerator Laboratory, P.O. Box 500, Batavia, IL 60510, USA \\ ${ }^{17}$ Department of Physics, University of Notre Dame, Notre Dame, IN 46556, USA \\ ${ }^{18}$ Department of Astrophysical Sciences, Princeton University, Princeton, NJ 08544, USA \\ ${ }^{19}$ Department of Physics and Astronomy, Rutgers University, 136 Frelinghuysen Road, Piscataway, NJ 08854, USA \\ ${ }^{20}$ Département de Physique Théorique and Center for Astroparticle Physics, Université de Genève, Quai E. Ansermet 24, CH-1211 Genève 4, Switzerland \\ ${ }^{21}$ Institut de Física d'Altes Energies, Universitat Autònoma de Barcelona, E-08193 Bellaterra (Barcelona), Spain \\ ${ }^{22}$ Institució Catalana de Recerca i Estudis Avançats, E-08010 Barcelona, Spain \\ ${ }^{23}$ Physics Department, Rochester Institute of Technology, Rochester, NY 14623, USA \\ ${ }^{24}$ Space Telescope Science Institute, 3700 San Martin Drive, Baltimore, MD 21218, USA \\ ${ }^{25}$ Department of Physics and Astronomy, Johns Hopkins University, 3400 North Charles Street, Baltimore, MD 21218, USA \\ ${ }^{26}$ Department of Astronomy and Astrophysics, The Pennsylvania State University, University Park, PA 16802, USA \\ ${ }^{27}$ Institute for Gravitation and the Cosmos, The Pennsylvania State University, University Park, PA 16802, USA \\ ${ }^{28}$ The Oskar Klein Centre, Department of Astronomy, AlbaNova, Stockholm University, SE-106 91 Stockholm, Sweden \\ ${ }^{29}$ National Astronomy Observatories, Chinese Academy of Science, Beijing 100012, China \\ Received 2012 September 14; accepted 2012 November 15; published 2013 January 14
}

\begin{abstract}
We present the cosmological analysis of 752 photometrically classified Type Ia Supernovae (SNe Ia) obtained from the full Sloan Digital Sky Survey II (SDSS-II) Supernova (SN) Survey, supplemented with host-galaxy spectroscopy from the SDSS-III Baryon Oscillation Spectroscopic Survey. Our photometric-classification method is based on the SN classification technique of Sako et al., aided by host-galaxy redshifts $(0.05<z<0.55)$. SuperNova ANAlysis simulations of our methodology estimate that we have an SN Ia classification efficiency of $70.8 \%$, with only 3.9\% contamination from core-collapse (non-Ia) SNe. We demonstrate that this level of contamination has no effect on our cosmological constraints. We quantify and correct for our selection effects (e.g., Malmquist bias) using simulations. When fitting to a flat $\Lambda \mathrm{CDM}$ cosmological model, we find that our photometric sample alone gives $\Omega_{m}=0.24_{-0.05}^{+0.07}$ (statistical errors only). If we relax the constraint on flatness, then our sample provides competitive joint statistical constraints on $\Omega_{m}$ and $\Omega_{\Lambda}$, comparable to those derived from the spectroscopically confirmed Three-year Supernova Legacy Survey (SNLS3). Using only our data, the statistics-only result favors an accelerating universe at $99.96 \%$ confidence. Assuming a constant $w \mathrm{CDM}$ cosmological model, and combining with $H_{0}$, cosmic microwave background, and luminous red galaxy data, we obtain $w=-0.96_{-0.10}^{+0.10}, \Omega_{m}=0.29_{-0.02}^{+0.02}$, and $\Omega_{k}=0.00_{-0.02}^{+0.03}$ (statistical errors only), which is competitive with similar spectroscopically confirmed SNe Ia analyses. Overall this comparison is reassuring, considering the lower redshift leverage of the SDSS-II SN sample $(z<0.55)$ and the lack of spectroscopic confirmation used herein. These results demonstrate the potential of photometrically classified SN Ia samples in improving cosmological constraints.
\end{abstract}

Key words: cosmology: observations - distance scale - supernovae: general - surveys

Online-only material: color figures 


\section{INTRODUCTION}

Supernovae (SNe) have historically been classified based on their optical spectroscopic properties (e.g., Filippenko 1997). Type Ia supernovae ( $\mathrm{SNe}$ Ia) are distinguished from other classes of SNe by their lack of hydrogen and helium spectral features, and the presence of other spectral features such as Si II absorption at rest-frame wavelength $6150 \AA$. This particular optical classification is unique, as it efficiently separates two distinct $\mathrm{SN}$ physical processes. The progenitors of $\mathrm{SNe}$ Ia are white dwarfs (WDs), unlike other common categories of $\mathrm{SNe}$, which result from the core collapse of stars with initial mass $M \gtrsim 8 M_{\odot}$. As the progenitors for core-collapse (non-Ia) $\mathrm{SNe}$, such as Type II SNe, are more luminous, the progenitor star has been identified from archival images on multiple occasions (Smartt 2009), while there have not yet been any direct observations of the WD progenitor of SNe Ia (though deep limits do exist on SN 2011fe; Li et al. 2011).

However, the lack of $\mathrm{H}$ and $\mathrm{He}$ in the spectrum, the composition of the ejecta, and the energy released in the explosion all strongly indicate that $\mathrm{SNe}$ Ia are the visible manifestation of a thermonuclear runaway explosion in a carbon-oxygen WD as its mass approaches the Chandrasekhar limit (Hoyle \& Fowler 1960; Nomoto 1982; Iben \& Tutukov 1984). The exact nature of the binary progenitor system (a single degenerate object accreting mass from a companion, or the merger of two WDs) has long been an open question. Recent observations have shown that both channels can lead to SNe Ia (Nugent et al. 2011; Bloom et al. 2012; Dilday et al. 2012), making the relative prevalence of each channel of primary concern among progenitor studies.

For over two decades SNe Ia have been of interest to cosmologists as distance indicators, as they display less dispersion at their peak magnitude than other classes of SNe and have high optical luminosities $\left(>10^{9} L_{\odot}\right)$. However, SNe Ia are not simple "standard candles," and the usefulness of SNe Ia is greatly enhanced by our ability to standardize their magnitudes. The discovery that photometric properties of SNe Ia, such as the light-curve width (Phillips 1993) and color (Riess et al. 1996; Tripp 1997), are correlated with the absolute magnitude at peak allowed for accurate distance measurements using $\mathrm{SNe}$ Ia, reducing the dispersion in the measured distance modulus $(\mu)$ to $\sim 0.14$ mag. This work led directly to the discovery that highredshift SNe Ia appear fainter than expected unless the expansion of the universe is accelerating (Riess et al. 1998; Perlmutter et al. 1999).

Over the last decade SN surveys have evolved, spanning greater fractions of the sky and discovering $\mathrm{SNe}$ at higher redshifts, with better photometric calibration and an improved understanding of systematic uncertainties. Due to the differing observational requirements necessary for monitoring SNe Ia over a wide range of redshifts (survey depth, area, wavelength coverage, etc.), the redshift-distance relationship, or "Hubble diagram," for SNe Ia is comprised of data from a number of surveys. The Hubble Space Telescope Program (GOODS SN sample; Riess et al. 2004, 2007), Supernovae Cosmology Project (SCP or HST Cluster SN sample; Knop et al. 2003; Kowalski et al. 2008; Amanullah et al. 2010; Suzuki et al. 2011), the Supernova Legacy Survey (SNLS; Guy et al. 2010), and Equation of State: SupErNovae trace Cosmic Expansion (ESSENCE; Miknaitis et al. 2007) provide nearly all SNe Ia measurements at $z>0.4$, with the first two surveys dominating at $z>0.9$.

\footnotetext{
${ }^{30}$ Clay Fellow.
}

The Sloan Digital Sky Survey II Supernova Survey (SDSS-II SN survey; Frieman et al. 2008; Kessler et al. 2009a) has populated the Hubble diagram at intermediate redshifts $(0.1<$ $z<0.4)$. Panoramic Survey Telescope \& Rapid Response System (PanSTARRS) is currently finding thousands of SNe Ia in the same redshift range. The largest of the low-redshift surveys include the Harvard-Smithsonian Center for Astrophysics SN group (Hicken et al. 2009a), the Nearby Supernova Factory (SNfactory; Aldering et al. 2002), the Carnegie Supernova Project (CSP; Hamuy et al. 2006; Folatelli et al. 2010), the Lick Observatory Supernova Search (Ganeshalingam et al. 2010), the Palomar Transient Factory (Law et al. 2009), and the Catalina Real-Time Transient Survey (Drake et al. 2009). Using a combination of data from different SN surveys, spanning the full range of redshifts available, the most recent cosmological analysis using only $\mathrm{SNe}$ Ia finds $\Omega_{\mathrm{m}}=0.18_{-0.10}^{+0.08}$ (stats) \pm 0.06 (sys) and $w=-0.90_{-0.20}^{+0.16}$ (stats) ${ }_{-0.14}^{+0.07}$ (sys) (Conley et al. 2011), under the assumption of a flat universe and a constant equation of state of dark energy $(w)$.

A common theme across most previous SN cosmology surveys is that SN spectroscopy is used only to classify the SN event and obtain a redshift (with the exception of the SNfactory and, in some cases, the SNLS; Bronder et al. 2007; Walker et al. 2010; Ellis et al. 2008). The distance to each object classified as an SN Ia is then determined from the multi-color time series photometry using one or more light-curve fitting models, e.g., SALT2 (Guy et al. 2007), MLCS2k2 (Riess et al. 1996; Jha et al. 2007), and SiFTO (Conley et al. 2008). Methods for constraining the absolute magnitude of an observed $\mathrm{SN}$ based on spectroscopic line ratios have also been developed and applied to nearby SNe (Foley et al. 2008; Bailey et al. 2009; Chotard et al. 2011; Nordin et al. 2011; Blondin et al. 2012; Silverman et al. 2012), though as of yet they have not been tested over a cosmologically interesting redshift range.

In the future, however, taking spectra of a large number of high-redshift $\mathrm{SNe}$ for classification purposes will be challenging, and could potentially limit the size and usefulness of any new survey. This will be the case for the Large Synoptic Survey Telescope (LSST Science Collaboration et al. 2009), where the complete spectroscopic classification of all its SN candidates will be simply impossible, and thus other methods need to be employed to utilize these huge SN programs. This challenge is already being confronted by the latest SN surveys, such as PanSTARRS, which have devised new and innovative techniques for classifying their SN candidates using only photometric imaging data (thus far tested on a small subset; see Scolnic et al. 2009). The SN survey from the Dark Energy Survey (DES) will probably not be able to spectroscopically classify all of their expected $\simeq 4000$ high-quality, high-redshift SNe Ia (Bernstein et al. 2012).

An alternative is to use photometric-only classification techniques. This idea is not new-Pskovskii (1977) proposed classifying SNe based on their observed decline rate-and even early SN Ia cosmology results included a significant fraction of highredshift events that lacked spectroscopic identification (Riess et al. 1998, 2004; Perlmutter et al. 1999; Tonry et al. 2003). One of the primary scientific drivers for developing photometric classifiers has been to aid in the spectroscopic follow-up of SN surveys, thus allowing them to use their spectroscopic resources more efficiently (Sullivan et al. 2006; Sako et al. 2008). However, making a Hubble diagram solely from photometrically classified $\mathrm{SNe}$ requires a lower false-positive rate (i.e., contamination by non-Ia $\mathrm{SNe}$ ) than does spectroscopic target selection 
from photometrically classified SNe. Photometry-only Hubble diagrams were introduced by Barris \& Tonry (2004), and have been presented more recently by Rodney \& Tonry (2010) and Bazin et al. (2011).

Most photometric-classification methods fit observed light curves to templates of different SN types and determine the likelihood of each class. These methods (e.g., Poznanski et al. 2002, 2007; Sullivan et al. 2006; Johnson \& Crotts 2006; Kuznetsova \& Connolly 2007; Kunz et al. 2007; Rodney \& Tonry 2009; Gong et al. 2010; Falck et al. 2010; Sako et al. 2011) typically remove SNe that resemble non-Ia SN templates based on their likelihoods, although there is considerable variety in the details. While Hlozek et al. (2012) (based on Kunz et al. 2007 and further developed by Newling et al. 2011; Knights et al. 2012) use templates to compute likelihoods for the type of each SN, they do not remove any objects from their cosmological fit. Rather, they use the likelihoods of each SN as a weight, retaining all possible information while computing an unbiased cosmology in a Bayesian manner. Higher level statistical analyses have also been applied to this problem, with a goal of finding a lower dimensional parameter space where a cleaner separation exists between the different types, thus simplifying the classification problem. Examples of these approaches include semi-supervised learning techniques such as diffusion maps (Richards et al. 2012), and kernel Principal Component Analysis applied to SN light curves (Ishida \& de Souza 2012).

In anticipation of the SN typing requirements to be encountered by DES, Kessler et al. (2010b, hereafter K10a) issued the "Photometric Supernova Classification Challenge," providing simulated light curves of different SN types based on a realistic DES-like SN survey and a training sample where the true SN type was given. The results of this challenge are presented in Kessler et al. (2010a, hereafter K10b), and provide some interesting insights into the relative performance of different SN classifiers. Overall, several different classification strategies produce similarly high scores in terms of both efficiency and contamination, but all proved subject to significant level of contamination $(\sim 20 \%)$. A problem that is common for many methods is that they require a training set of known SN types. If this set is biased and not representative of the whole sample then the classification will be biased as well, an effect that was seen in the K10a challenge.

In this paper we build upon the photometric-classification algorithm of Sako et al. (2011, hereafter S11), which obtained the highest overall Figure-of-Merit (FoM) in K10b. We use here the full three-year data set from the SDSS-II SN Survey, including a new collection of host-galaxy redshifts obtained by the SDSS-III Baryon Oscillation Spectroscopic Survey (BOSS; Eisenstein et al. 2011; Dawson et al. 2013).

We optimize selection cuts and determine the biases of our new method with extensive simulations using the SuperNova ANAlysis (SNANA; Kessler et al. 2009b) software package, and apply redshift-dependent corrections to our data. We show that photometric classification can provide SN Ia samples with low contamination and well-understood biases, and present cosmological constraints that are competitive with those derived from existing spectroscopic samples.

The paper is organized as follows. In Section 2 we detail the SN and host-galaxy data that we analyze in this paper. In Section 3 we discuss our SN classifier, with emphasis on the light-curve fitter and selection criteria. We perform a rigorous analysis of and derive corrections for biases introduced by our selection criteria, shown in Section 4. In Section 5 we present our full photometric Hubble diagram and consistency checks. Cosmological constraints from our photometrically derived sample are in Section 6 along with comparisons to other spectroscopic cosmological fits. In Section 7 we discuss our results, how this analysis could be improved, and how our work applies to upcoming large-scale SN surveys. Finally in Section 8 we detail the main conclusions of this paper.

\section{DATA}

\subsection{The SDSS-II Supernova Survey}

The SDSS-II SN Survey is a dedicated search for intermediate-redshift $\mathrm{SNe}$ from repeated scans of the equatorial "Stripe 82" region (covering $\simeq 300 \mathrm{deg}^{2}$ ) of the original SDSS (York et al. 2000; Frieman et al. 2008). For three months a year (September-November) over a three-year period (2005-2007), the SDSS telescope (Gunn et al. 2006; Gunn et al. 1998) performed multi-color ugriz imaging (Fukugita et al. 1996; Gunn et al. 2006; Ivezić et al. 2007; Doi et al. 2010) of this area of sky, with a cadence of a few times per week. The SDSS uses asinh magnitudes (Lupton et al. 1999), although SDSS is on the AB system after applying small offsets (see Section 2.2.1). The analysis of SDSS astrometry is described in Pier et al. (2003).

This multi-epoch data were then used to identify SN Ia candidates in real-time for further spectroscopic observations (Sako et al. 2008), resulting in over 500 spectroscopically confirmed SNe Ia (Zheng et al. 2008; Ostman et al. 2012; Konishi et al. 2011; Foley et al. 2012). Well-observed subsamples of these intermediate-redshift SNe have been used in a variety of studies, primarily focused on constraining cosmology (Kessler et al. 2009a; Sollerman et al. 2009; Lampeitl et al. 2010a), the measurement of SN rates (Dilday et al. 2010; Smith et al. 2012), and the study of host-galaxy properties and their correlations with SNe Ia (Lampeitl et al. 2010b; Gupta et al. 2011; D'Andrea et al. 2011; Galbany et al. 2012). The full three-year SDSS-II SN Sample will be published in M. Sako et al. (in preparation).

The real-time spectroscopic sample of SDSS-II SNe is incomplete and potentially biased, as a function of redshift. This bias comes from a number of different, and sometimes competing, effects and is therefore hard to predict a priori and thus correct for. First, the decisions made by observers following up $\mathrm{SN}$ candidates were based on the local weather conditions (at a variety of telescopes), the position of the $\mathrm{SN}$ candidates on the sky, and the location of the SN candidate in the host galaxy. This is illustrated in Table 2 of Smith et al. (2012) where the spectroscopic completeness of the SDSS-II SN Survey drops below $40 \%$ at $z>0.4$ (see also Kessler et al. 2009a).

Second, our targeting of SN candidates gave priority to events in red elliptical host galaxies (see Equation (7) of Sako et al. 2008), as these SNe are likely to be less affected by dust in their host galaxy. This prioritization is seen in the spectroscopically confirmed SNe Ia, which have lower reddening values than predicted from simulations without spectroscopic selection (Figure 16, Kessler et al. 2009a). However, the rate of SNe Ia in red elliptical galaxies is lower than seen in blue, star-forming spiral galaxies (Mannucci 2005; Wang et al. 1997; Smith et al. 2012), so this additional upweighting given to the $\mathrm{SNe}$ Ia in red ellipticals might be a subdominant effect. As will be shown in Figure 20 of this paper, the host galaxies of spectroscopically confirmed SNe Ia are, on average, representative of the whole population of host-galaxy colors studied in our BOSS sample 
(see Section 4.2). However, it is clear that the spectroscopically confirmed SNe Ia from the SDSS-II SN Survey are biased in absolute magnitude with respect to the entire SN Ia population. To avoid any such biases, we do not use spectroscopic SN information anywhere in our photometric classification (as discussed in Section 3). Any spectroscopically confirmed SNe Ia that fail our photometric criteria are not included in our final sample to preserve selection consistency.

\subsection{BOSS Ancillary Targets}

We undertook a BOSS ancillary spectroscopic program (see Dawson et al. 2013 for details of these programs), to obtain host-galaxy information for most SDSS-II SN candidates. We aim to obtain a larger, and more complete, SN Ia sample from the SDSS-II SN Survey through photometric classification. We also took the opportunity to target the host galaxies of a range of other, possibly interesting, transient events detected as part of the SDSS-II SN Survey. Such a project was well suited to the small number of ancillary targets available in each BOSS spectroscopic field and would have been impossible to achieve on a normal instrument (either through queue scheduling or normal observer mode), because of the combination of the low surface density of faint targets (between 5 and 25 targets per deg ${ }^{2}$ to $r \sim 22$ ) spread over a wide area ( $\simeq 300 \mathrm{deg}^{2}$ of "Stripe 82"). Table 1 of Frieman et al. (2008) shows that the SDSS-II SN Survey used over $1000 \mathrm{hr}$ (or $\sim 100$ nights) of telescope time between 2005 and 2006 to spectroscopically confirm over 350 SNe Ia, utilizing many of the larger optical telescopes in the world (HET, NTT, NOT, APO, Subaru, WHT, SALT, Keck, NOAO, TNG). It is hard to envisage how we could have used such resources to target thousands of host galaxies as presented herein.

In detail, we obtained spectroscopic observations using the BOSS spectroscopic system (Smee et al. 2012) of 3761 galaxies spread almost evenly across the "Stripe 82" region. These targets were chosen using two selection algorithms (described below) which were complementary, but different, in their scientific objectives. The first algorithm focused on improving cosmological constraints from SNe Ia in the SDSS-II SN Survey by obtaining a sample free from the possible spectroscopicselection biases discussed above. The other algorithm targeted interesting subsamples of transients detected as part of the SDSS-II SN Survey. In this paper we focus exclusively on the first of these objectives, but include in our analysis galaxies, and their associated SNe, observed by BOSS originating from either algorithm.

\subsubsection{Algorithm One: Additional Type Ia Candidates}

We targeted galaxies that hosted an SN event of any type, based on object classifications using the "Photometric SN IDentification" (PSNID) method of S11, which we describe in detail in Appendix A. We applied PSNID to the multicolor (ugriz) light-curve data from SDSS-II created with the Scene-Modeling Photometry (SMP) method of Holtzman et al. (2008), ${ }^{31}$ assuming a flat prior on all SN parameters. We do not include any spectroscopic information for these objects, and

\footnotetext{
31 Improvements in this photometry compared to that used in Kessler et al. (2009a) include the discovery of a small $(\sim 10 \%)$ correction for an underestimate of uncertainties for low-flux data, and new (2011 December) $\mathrm{AB}$ offsets from the SDSS native magnitudes system of

$\Delta u=-0.066, \Delta g=0.021, \Delta r=0.005, \Delta i=0.020$, and $\Delta z=0.013$. Details of this re-calibration can be found in M. Betoule et al. (in preparation) and M. Sako et al. (in preparation).
}

thus place a flat prior on the SN redshift as well. We select transient events that were classified as likely $\mathrm{SNe}$ of any type, i.e., probable SN Ia, SN II, or SN Ibc.

For all these candidates, we visually inspected the SDSS images (from the DR7 Skyserver; Abazajian et al. 2009) of the three nearest galaxies to each transient and manually assigned the most likely host galaxy for each candidate. In the majority of cases $(95 \%)$ the nearest galaxy on the sky (angular separation) to the candidate was classified as the host, but in some cases the nearest galaxy was either clearly a background object or a star misclassified as a galaxy. In these cases we classified either the second (4\%) or third (1\%) nearest galaxy as the most likely host. Taking into account the observational limitations of the program, we gave priority to host galaxies with a fiber magnitude brighter than $r_{\text {fiber }}=21.25$, based on SDSS-I/II photometry. However, to fully utilize our allocation of BOSS fibers, we also include a small subsample of host galaxies fainter than this limit. Combined, these samples made up our main target list of 2781 galaxies.

In 66 cases, the nearest object to the $\mathrm{SN}$ event was classified as a faint star in the SDSS DR7 database, but was clearly a galaxy as seen in the SDSS images. For these objects, we targeted the nearest "stellar" source in the DR7 database, which could have a fainter fiber magnitude than our main BOSS targets $\left(r_{\text {fiber }}<21.25\right)$ and were given lower priority for BOSS observations.

Finally, we cross-referenced the whole target list with the SDSS DR7 database and found 276 of these host galaxies had a spectrum already. For these cases we targeted the location of the SN event rather than placing the BOSS fiber at the center of the galaxy, as was done in all other cases and in the original SDSS-I/II survey. The motivation for this fiber placement is that galaxy spectra at the location of the SN could be useful for studies into correlations between SN properties and the environment in which the SN occurs (Gallagher et al. 2005, 2008; Gupta et al. 2011; D'Andrea et al. 2011; Galbany et al. 2012). As our focus here is only on obtaining redshifts to aid in classifying transients, these spectra (at the location of the $\mathrm{SN}$ event) serve our purposes equally well. In these cases, we ignored the fiber magnitude of the host galaxy (calculated at the center of the galaxy) and observed the SN location position regardless of our $r_{\text {fiber }}=21.25$ limit for the main sample.

\subsubsection{Algorithm Two: Random Sample of Additional Transients}

The goals of our second sample were both to study our overall selection biases (e.g., determine the effect of active galactic nuclei, AGNs) and to further study interesting and unusual variable objects observed by SDSS-II e.g., hydrogenpoor, superluminous SNe (Quimby et al. 2011; Leloudas et al. 2012). Achieving the first of these goals requires an unbiased sample of non-SN transient host galaxies, created by choosing at random from the set of non-SN transients in the magnitude range of $19.5<r<21.5$. We imposed no magnitude limit on the host galaxies of these targets, of which there were 980; however, we did require the galaxy to be detected in the DR7 galaxy catalog.

As with the SN host-galaxy sample in Section 2.2.1, we visually inspected the three nearest galaxies in DR7 to each transient, selecting as our BOSS target the most likely host to the transient. This proved to be the nearest galaxy (angular separation) in the vast majority $(99.4 \%)$ of cases. The high percentage of host galaxies being matched to the nearest galaxy, compared to the previous sample in Section 2.2.1, is likely due 
to these transients being quasars that are located in the cores of galaxies, as opposed to $\mathrm{SNe}$, which can be located throughout the galaxy. The targets from this sample were given the lowest priority for observation when assigning BOSS fibers.

Another key difference between Algorithms One and Two was that in Algorithm Two transient events were allowed to show variability over multiple years, or have light curves that failed in the initial phototyping. Targets selected by Algorithm One were detected in only one season of the SDSS-II SN Survey.

\subsection{Reduction of BOSS Spectra}

Our ancillary targets described above were merged with other ancillary BOSS targets on the "Stripe 82" region and observed together as part of the normal SDSS-III observing program during 2009 and 2010 (see Eisenstein et al. 2011; Dawson et al. 2013, for a description of BOSS observations and programs). Some of our targets were lost at this stage because of BOSS fiber collision issues, i.e., two objects within $55^{\prime \prime}$ of each other cannot be observed on a single BOSS spectroscopic plate.

By the end of 2010 all BOSS plates on "Stripe 82," including most of our ancillary targets, had been observed. For the purpose of this paper we are only interested in the galaxy redshift measurements (and their errors). The details of the spectral reductions of our sample of BOSS SN host-galaxy spectra can be found in M. D. Olmstead et al. (in preparation), and the redshift and object classification of BOSS spectra in general is described in Bolton et al. (2012). In brief, our sample was processed using the standard BOSS spectroscopic analysis software, which is based on the original SDSS-I/II reduction pipelines. This pipeline has at least a $95 \%$ success rate in obtaining redshifts for spectra from the primary galaxy sample (e.g., Anderson et al. 2012). M. D. Olmstead et al. (in preparation) carried out a detailed comparison between the BOSS pipeline redshifts and those produced, and manually inspected, using the publicly available AAO RUNZ software. All the spectral data from our SN host-galaxy ancillary program are now public as part of the SDSS DR9 data release (Ahn et al. 2012).

After removing spectra with low-redshift confidence, low signal-to-noise ratio $(\mathrm{S} / \mathrm{N})$, and large redshift errors, our SDSS-II SN ancillary program on BOSS produced 3323 reliable redshifts. This sample is composed of 2382 likely SN host galaxies from the 2781 targets selected via Algorithm One in Section 2.2.1, and 941 from the random sample of 980 galaxies chosen in Section 2.2.2. The spectroscopic target efficiency from Algorithm One is $86 \%$, which is approximately $10 \%$ lower than for Algorithm Two. This difference is due to two subsamples in Algorithm One that have a lower efficiency, probably due to the lack of an imposed fiber-magnitude limit in these cases. The first of these subsamples targets the nearest photometric object to the SNe when there was some ambiguity about the star/ galaxy separation; this had an efficiency of only $27.3 \%$, but was a very small subsample. The second subsample was created using less stringent cuts on the quality of the light curve (i.e., lower $\mathrm{S} / \mathrm{N}$ ) to provide an additional list of probable SN locations; this subsample has a lower average host-galaxy fiber magnitude $\left(\left\langle r_{\text {fiber }}\right\rangle=22.17\right)$ than the main sample $\left(\left\langle r_{\text {fiber }}\right\rangle=20.62\right)$, which leads to a lower redshift efficiency $(70.1 \%)$.

At this point, we checked the SDSS DR8 database for any host galaxies that failed to gain a redshift from our BOSS observations and reductions, based on either Algorithm One and Two as described above. We found an additional 178 host galaxies had a successful redshift measurement in DR8, and add them to our final BOSS sample. We note that most of these

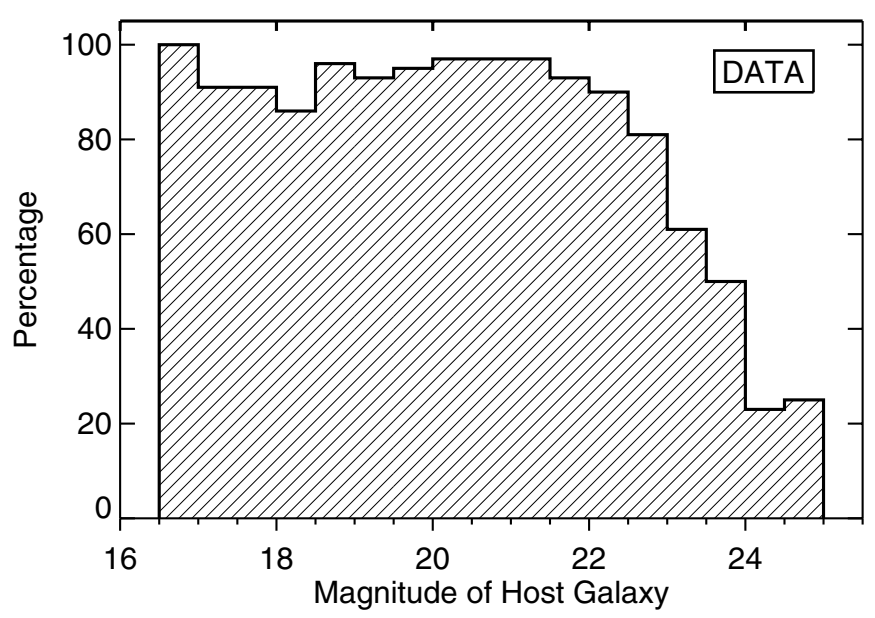

Figure 1. Percentage of well-measured (based on comparison with RUNZ and visual inspection) redshifts obtained as a function of the $r$-band fiber magnitude from the photometry of the SNe (Algorithm One) and general transients (Algorithm Two) host galaxies. We recover 3323 well-measured redshifts from the 3761 galaxies observed by our BOSS program.

galaxies were on our BOSS target list, but were not observed during our ancillary program. This combined sample (which, for simplicity, we will continue to call our "BOSS" sample) forms the basis for our subsequent re-analysis of the SDSS-II SN light-curve data in Section 3, now with the SN candidate redshift constrained to match the observed host galaxy.

We present in Figure 1 the percentage of successful redshift measurements (from comparing with RUNZ and visual inspection) obtained from our BOSS-observed host galaxies, shown as a function of the host-galaxy $r$-band fiber magnitude. Figure 2 shows the measured redshift distribution for our BOSS targets, grouped by their target algorithm. For comparison we also show in Figure 2 the spectroscopically confirmed SNe Ia from SDSS-II, which clearly peak at lower redshifts. There are a significant number of transient host galaxies with redshifts greater than 0.5 , extending out to $z \approx 1$, which are primarily quasars. In Figure 3 we display a few example BOSS spectra of our SN host galaxies, spanning the full redshift range of SDSS-II $\mathrm{SNe}$. Though there is a wide range of $\mathrm{S} / \mathrm{N}$ in these spectra, spectral features (particularly the $4000 \AA$ break and emission lines), which allow us to measure the galaxy redshift, are clearly visible.

\section{CREATING A PHOTOMETRIC HUBBLE DIAGRAM}

In this section, we describe our construction of a photometrically classified SN Ia Hubble diagram. We provide details of the SN simulations used to determine the optimal selection criteria (Section 3.1) and apply these to the data (Section 3.2). We describe the resulting Hubble diagram in Section 3.3.

\subsection{Selection Criteria}

Here we define, using simulations, the criteria by which we construct our photometric SN Ia Hubble diagram. Our primary focus is to minimize the contamination in our sample from non-Ia $\mathrm{SNe}$; this is one of the major concerns associated with measuring cosmological parameters with photometrically classified SNe, and has the potential to introduce significant systematic errors in the cosmological analysis (Section 6). Given the large number of SN candidates included in our data set, the conservative classification criteria that we seek will still result in statistical errors on our cosmology smaller than those due to systematics (Appendix D). By focusing primarily on the purity 


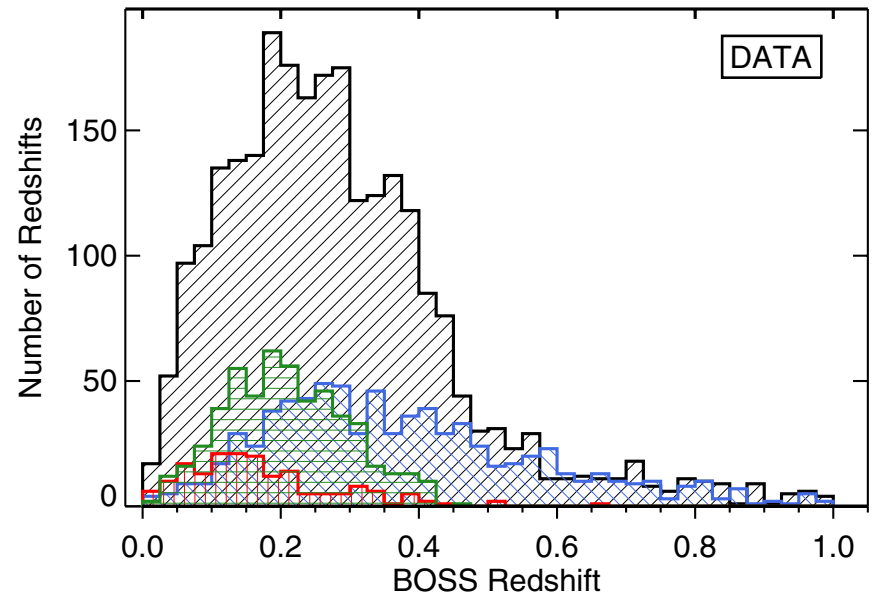

Figure 2. Redshift distribution of the targets observed with BOSS. The black histogram is the subset which was selected using Algorithm One (candidate SN host galaxies) and the blue is the from Algorithm Two (host galaxies for general transients) as discussed in Section 2. The green histogram shows all spectroscopically confirmed SNe Ia from the SDSS-II SN Survey. The red histogram shows the additional 177 SDSS-II DR8 host-galaxy redshifts.

(A color version of this figure is available in the online journal.)

of our sample we have sacrificed its overall completeness, and therefore we caution the reader against using this particular sample for analyses that require high completeness (e.g., SN rate measurements).

There are three types of criteria that we apply to our sample. First, we apply "light-curve quality" cuts, which are criteria that ensure the SN light curves (real or simulated) are of sufficient quality to be well-fit with SALT2 (see Appendix B for details) to give accurate distance moduli and provide meaningful typing constraints. Next, we consider the optimal values for the PSNID
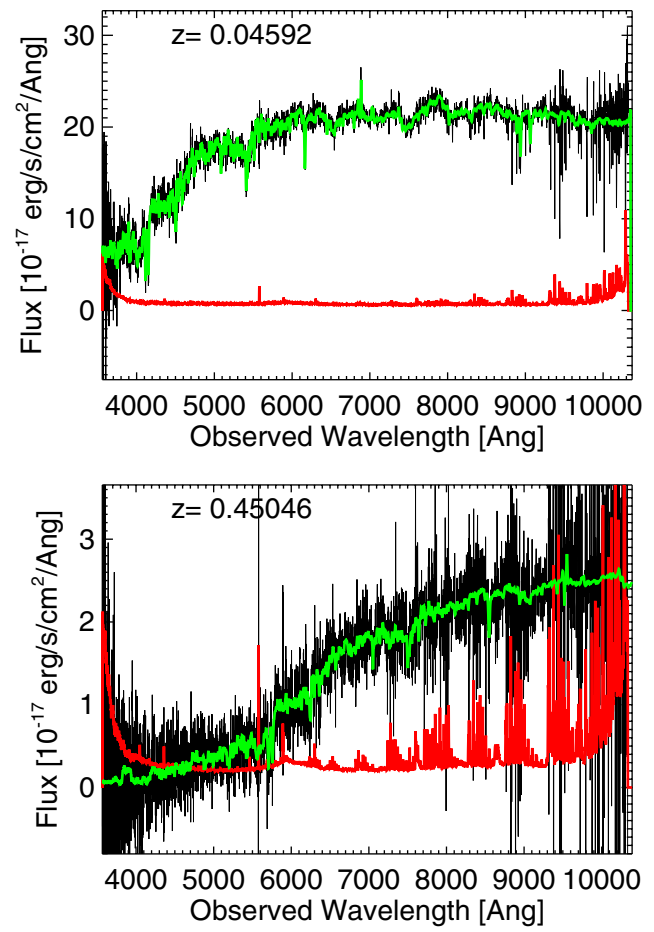

classification parameters (see Appendix A for details), which will differ from those used in S11 since we are both using an improved version of PSNID and wish to increase the recovered sample purity. Finally, we can further improve the purity of our sample by applying "color and stretch" criteria based on the derived SALT2 parameters for each light curve and our knowledge of the likely acceptable range for these properties for SNe Ia. We outline each of these types of criteria below, following a brief overview of the simulations and FoM we use to guide our criteria selection. In Table 1, we show the effect of each selection criterion as applied in the following sections.

\subsubsection{Supernova Simulations}

To test the purity and completeness of our sample, and help define the best selection criteria, we use the publicly available simulations of the SDSS-II SN Survey created as part of the "Photometric SN Classifier Challenge" of K10a. These simulations were made available via the SN challenge Web site $^{32}$ and were produced using the SNANA software (Kessler et al. 2009a), as described in K10b. We decided to use these simulations to test our completeness and purity as they have been well tested by many researchers, and were designed specifically for testing photometric classification of SNe. They also provide an accurate description of the conditions under which our data were acquired.

In detail, the simulations have 10 times the number of $\mathrm{SNe}$ as the full three-year SDSS-II SN Survey. They are based on realistic weather, seeing, and photometric zero-point variations, and have a realistic mixture of different $\mathrm{SN}$ types out to a redshift limit of $z=0.45$. The number of SNe Ia created in these simulations is based on the observed SN Ia redshift dependence from the SDSS-II SN Survey first-year rates analysis (Dilday

\footnotetext{
32 http://sdssdp62.fnal.gov/sdsssn/SIMGEN_PUBLIC
}
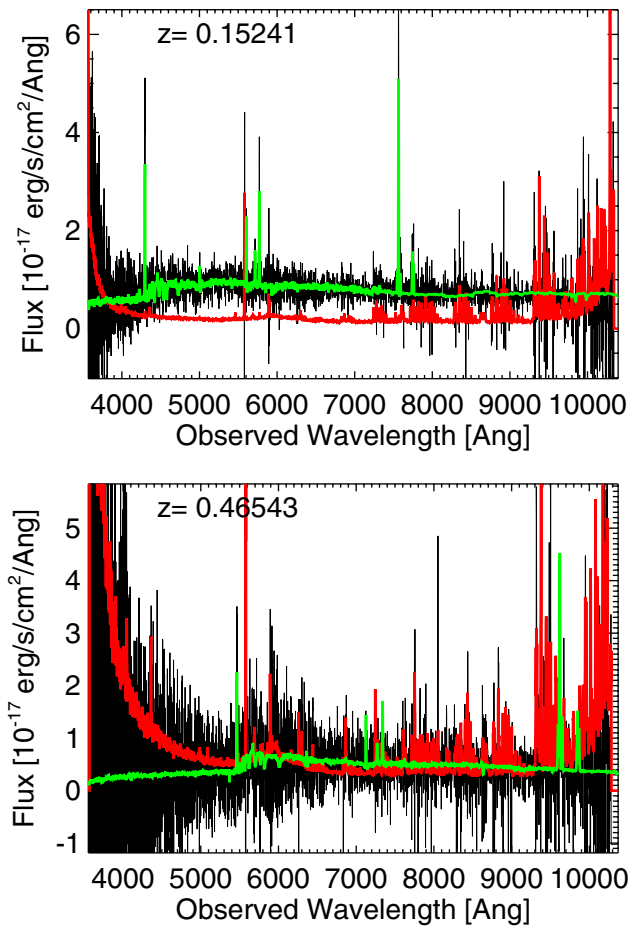

Figure 3. Galaxy spectra from BOSS. The top left panel is a low-redshift galaxy with a high S/N continuum, while the top right panel shows a galaxy with virtually no continuum, but several clear emission lines. Both bottom panels are at the high end of our redshift range $(z>0.4)$. The black lines show the data, the green is the best-fit eigenspectrum spectra, and the errors are in red (except masked points, which are set to zero).

(A color version of this figure is available in the online journal.) 
Table 1

Number of SNe in our Simulated Sample as a Function of the Selection Criteria Applied to the Data

\begin{tabular}{|c|c|c|c|c|c|c|}
\hline \multirow[t]{2}{*}{ Cut } & \multicolumn{3}{|c|}{ Classified SNe Ia } & \multirow{2}{*}{$\begin{array}{l}\text { Contam- } \\
\text { ination }\end{array}$} & \multirow{2}{*}{$\begin{array}{c}\text { SN Ia } \\
\text { Efficiency }\end{array}$} & \multirow{2}{*}{$\begin{array}{c}\text { FoM } \\
(W=5)\end{array}$} \\
\hline & Total & $\mathrm{SNe} \mathrm{Ia}$ & Non-Ia SNe & & & \\
\hline Number in simulation & 12203 & 5018 & 7185 & & & \\
\hline Light-curve quality & 9186 & 3734 & 5452 & $59.4 \%$ & $100 \%$ & 12.05 \\
\hline$P_{\mathrm{Ia}}>P_{\text {non-Ia }}($ PSNID $)$ & 6354 & 3701 & 2653 & $41.8 \%$ & $99.1 \%$ & 21.6 \\
\hline$\chi_{r}^{2}<1.2(\mathrm{PSNID})$ & 4737 & 3420 & 1317 & $27.8 \%$ & $91.6 \%$ & 31.3 \\
\hline$X_{1}$ and $c$ cut (SALT2) & 2918 & 2675 & 243 & $8.3 \%$ & $71.6 \%$ & 49.2 \\
\hline Color-magnitude criteria (SALT2) & 2750 & 2644 & 106 & $3.9 \%$ & $70.8 \%$ & 59.0 \\
\hline
\end{tabular}

Notes. In each row we show the cumulative effect of all the previous criteria on the contamination, efficiency, and FoM (assuming $W_{\text {Ia }}^{\text {false }}=5$ ).

et al. 2008), and the non-Ia SNe redshift dependence is based on the core-collapse rate analysis from SNLS (Bazin et al. 2009). The core-collapse contribution has been intentionally overestimated in the simulations in order to increase the statistics of non-Ia SNe that are misidentified as SN Ia, rather than underestimate the amount of non-Ia contamination.

For the analysis presented herein we only use the SALT2 simulated SN Ia light curves (ugriz) in this public data set (the simulations also included MLCS generated light curves). Since this is only half of the generated SNe Ia in the simulation, we only include half of the non-Ia SNe to keep the ratio of SNe Ia to non-Ia SNe correct. The SNe Ia light curves use the SALT2 standardization parameters of $\alpha=0.11$ and $\beta=3.2$, with an assumed intrinsic dispersion of $\sigma_{\text {int }}=0.12 \mathrm{mag}$. The intrinsic dispersion is included in the simulations of the SNe Ia by adding random color variations for each $\mathrm{SN}$ in each passband, drawn from a Gaussian distribution with $\sigma_{m}=0.09 \mathrm{mag}$, applied coherently to all SN epochs. The non-Ia SNe in the simulations are based on 41 well-measured spectroscopically confirmed non-Ia SNe templates. A flat $\Lambda$ CDM cosmology with $\Omega_{\mathrm{m}}=0.3$ and $H_{0}=70 \mathrm{~km} \mathrm{~s}^{-1} \mathrm{Mpc}^{-1}$ was also assumed.

We note that the simulations accurately model the SDSS-II SN Survey software detection pipeline, and thus any SNe that were too faint to be detected by the SDSS survey would not be included in the simulations. The simulations can also model spectroscopic selection, which we do not make use of in our main analysis but is included where we make checks against our spectroscopically confirmed subsample.

\subsubsection{Defining the Figure-of-Merit}

As we know the true types of $\mathrm{SNe}$ in the simulations, we can estimate the efficiency and purity of our photometric classifications as a function of our selection criteria. We use the definition of photometric typing efficiency $\epsilon_{\mathrm{Ia}}$ from S11,

$$
\epsilon_{\mathrm{Ia}}=\frac{N_{\mathrm{Ia}}^{\mathrm{true}}}{N_{\mathrm{Ia}}^{\mathrm{CUT}}},
$$

where $N_{\mathrm{Ia}}^{\mathrm{CUT}}$ is the total number of true SNe Ia in the simulations that pass the light-curve quality cuts (see Section 3.1.3), and $N_{\mathrm{Ia}}^{\text {true }}$ is the subsample of $N_{\mathrm{Ia}}^{\mathrm{CUT}}$ that are classified correctly (itself a function of additional cuts; see Table 1). As noted in S11, this equation measures the efficiency of classification only for well-observed SNe Ia, not the total efficiency of identifying all $\mathrm{SNe}$ Ia in our simulated data. From here on we refer to the parameter defined in Equation (1) as simply our efficiency.

We additionally define the weighted purity as in S11,

$$
\eta_{\mathrm{Ia}}=\frac{N_{\mathrm{Ia}}^{\text {true }}}{\left(N_{\mathrm{Ia}}^{\text {true }}+W_{\mathrm{Ia}}^{\text {false }} N_{\mathrm{Ia}}^{\text {false }}\right)},
$$

where $N_{\mathrm{Ia}}^{\text {false }}$ is the number of non-Ia SNe incorrectly classified as $\mathrm{SNe}$ Ia and $W_{\mathrm{Ia}}^{\text {false }}$ weights the contribution of misclassifications to the overall purity. This definition has the usual meaning of purity for $W_{\mathrm{Ia}}^{\text {false }}=1$, and for a given amount of contamination by non-Ia SNe a higher (lower) value of $W_{\mathrm{Ia}}^{\text {false }}$ results in a lower (higher) value of $\eta_{\text {Ia }}$.

The purity and efficiency of the photometric classification can be combined to form an FoM. As defined in S11 and K10b, the FoM is simply the product of Equations (1) and (2),

$$
\text { FoM }=\frac{N_{\mathrm{Ia}}^{\text {true }}}{N_{\mathrm{Ia}}^{\text {CUT }}} \frac{N_{\mathrm{Ia}}^{\text {true }}}{\left(N_{\mathrm{Ia}}^{\text {true }}+W_{\mathrm{Ia}}^{\text {false }} N_{\mathrm{Ia}}^{\text {false }}\right)} .
$$

The FoM in Equation (3) does not encapsulate information on the cosmology constraints, but rather is a simple metric that describes the broad merits of a classifier. Nevertheless, we aim to create a classification that optimizes this FoM with a suitably chosen weighting factor $\left(W_{\mathrm{Ia}}^{\text {false }}\right)$ that represents our previously stated choice of prioritizing purity over efficiency. The ideal choice for $W_{\mathrm{Ia}}^{\mathrm{fa}}$ ese is a complicated function of the contaminating objects, sensitive to the redshift and magnitude distribution of each subtype. This issue is not investigated in detail here; instead, we tested the effects of several different weighting values greater than one and empirically determined that $W_{\mathrm{Ia}}^{\text {false }}=5$ is the best choice, as it produced the sharpest peak in the FoM in Figures 4, 5, and 7. We therefore use this value in all subsequent analyses. For further discussion of the importance of purity in SN Ia samples for cosmological analyses, see Bernstein et al. (2012) and Gjergo et al. (2012), who discuss this issue in the context of the DES. We note that the efficiency, purity and FoM are only calculated after we have applied our light-curve quality cuts, as this is our baseline for defining SNe Ia that are potentially useful for cosmological constraints.

\subsubsection{Light-curve Quality Cuts}

We begin our analysis of the simulations by applying data quality cuts to all light curves, removing $\mathrm{SNe}$ that have insufficient epochs to provide any useful measurement. Defining $t$ as the rest-frame epoch (in days) of each SN relative to peak (determined from the best-fit PSNID SN Ia model), we require at least one epoch of photometry near peak at $-5<t<+5$ and at least one additional epoch at $t>15$, as in S11. However, we do not apply the S/N criteria outlined in $\mathrm{S} 11$ ( $\mathrm{S} / \mathrm{N}>5$ in at least two of the gri bands), as this could remove many high-redshift or underluminous SNe that may be identified through photometric classification. We note that an implicit $\mathrm{S} / \mathrm{N}$ limit does in fact exist, as the difference imaging software (sdssdiff) in the SDSS-II SN Survey requires multiple detections at $\mathrm{S} / \mathrm{N}>3$ 


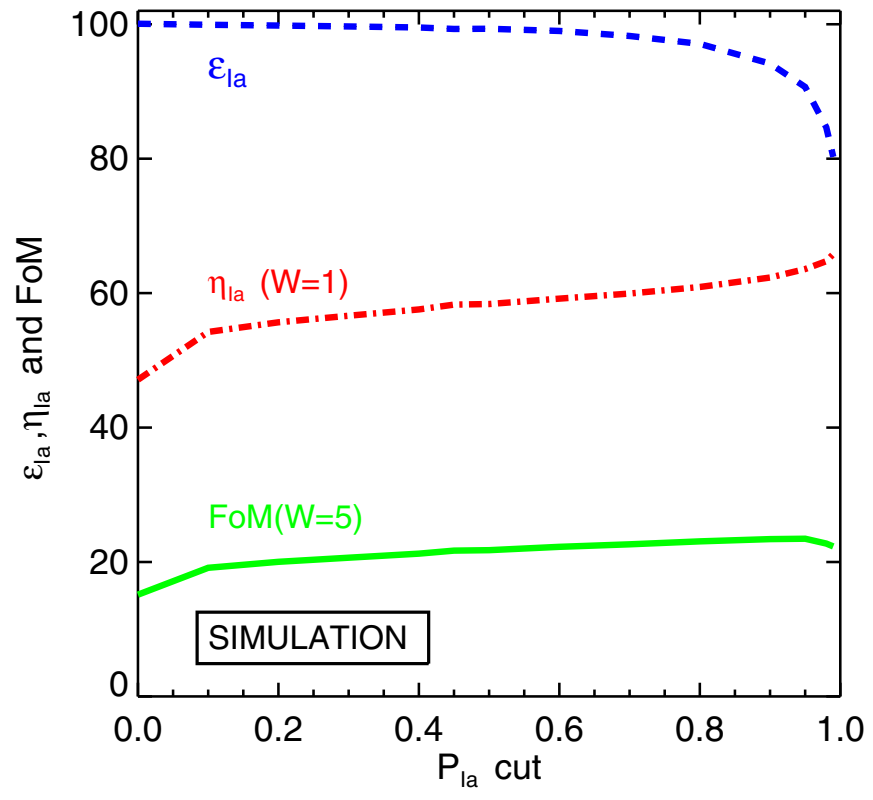

Figure 4. Efficiency (dashed blue line), purity (dot-dashed red line), and FoM (solid green line) for the simulated sample as a function of the position of the PSNID $P_{\text {Ia }}$ probability cut. We plot the true purity $\left(W_{\text {Ia }}^{\text {false }}=1\right)$, and only change the weighting factor to $W_{\mathrm{Ia}}^{\mathrm{false}}=5$ in the FoM.

(A color version of this figure is available in the online journal.)

for an object to be labeled an SN candidate in the first place (Sako et al. 2008). Finally, we check again that none of our SN candidates were detected in more than one of our SDSS-II SN search season.

Since we have no hard $\mathrm{S} / \mathrm{N}$ limit, it is not strictly true that our light-curve quality cuts remove all objects that are incapable of providing useful cosmological constraints from their light curves. The simplicity of our criteria is an attempt to balance the necessity of sufficiently useful data with the desire to be unbiased against faint objects.

\subsubsection{PSNID Criteria}

We run PSNID (described in Appendix A) on all simulated light curves that pass our light-curve quality cuts (Section 3.1.3), placing flat priors on $A_{V}$ (the host-galaxy extinction), $T_{\max }$ (the time of peak brightness), and $\Delta m_{15}$ (the stretch parameter). We use the true redshift of each $\mathrm{SN}$ as a prior, with an uncertainty on $z$ of the measured error.

We investigate PSNID criteria for removing non-Ia SNe using our simulations, examining their effect on the efficiency, purity, and FoM. First, we optimize the cut on the PSNID probability of being an SN Ia $\left(P_{\mathrm{Ia}}\right)$. In Figure 4 we show the efficiency, purity, and FoM as a function of $P_{\text {Ia }}$. Due to the general behavior of PSNID, which tends to cluster values of $P_{\mathrm{Ia}}$ around zero or one (demonstrated in Figure 7 of S11), these functions are relatively flat. As $P_{\mathrm{Ia}}$ does not provide much discriminating power beyond these extreme values, the FoM has little sensitivity to the $P_{\text {Ia }}$ cut value. We thus require $P_{\mathrm{Ia}}>P_{\mathrm{Ibc}}$ and $P_{\mathrm{Ia}}>P_{\mathrm{II}}$ for inclusion in our SN Ia sample, which combines high efficiency with modest purity. For reference, this is a less constrained criterion than S11, which adopted $P_{\text {Ia }}>0.9$.

We also use the reduced chi-squared $\left(\chi_{r}^{2}\right)$ of the best-fit PSNID model as a discriminator of non-Ia SNe, and determine the optimal value for this cut after the PSNID cut $\left(P_{\mathrm{Ia}}>P_{\mathrm{Ibc}}\right.$ and $\left.P_{\mathrm{Ia}}>P_{\mathrm{II}}\right)$ is applied. In Figure 5 one can see a clear peak in the FoM at $\chi_{r}^{2} \simeq 1.2$, close to where the purity and efficiency

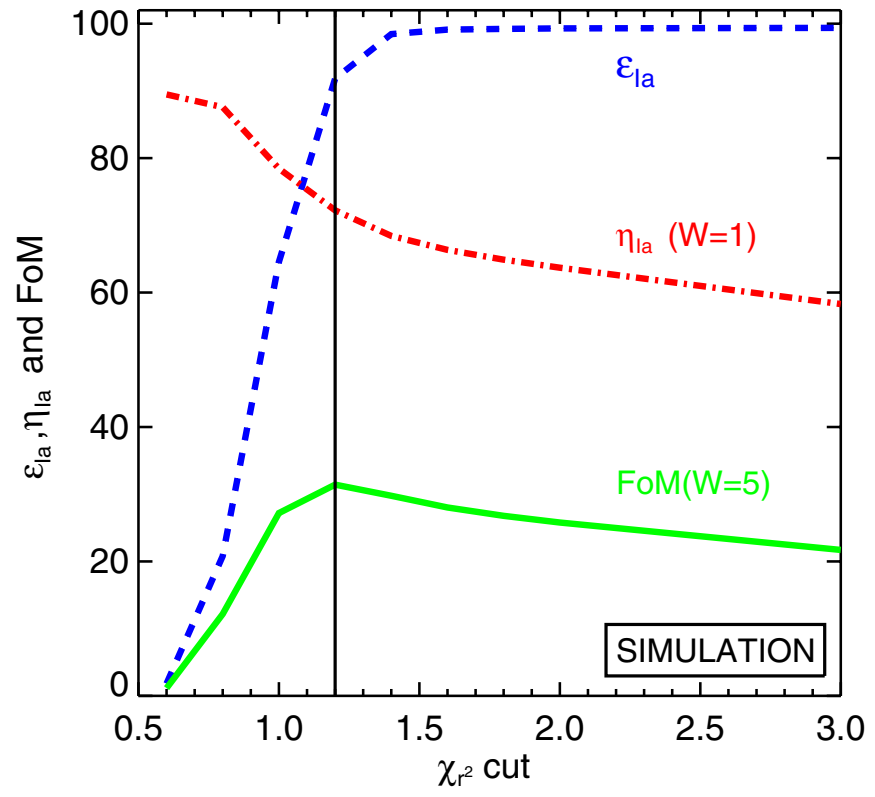

Figure 5. Efficiency (dashed blue line), purity (dot-dashed red line), and FoM (solid green) for the simulated sample as a function of $\chi_{r}^{2}$, after the PSNID cut $\left(P_{\mathrm{Ia}}>P_{\mathrm{Ibc}}\right.$ and $\left.P_{\mathrm{Ia}}>P_{\mathrm{II}}\right)$ was applied. We plot the true purity $\left(W_{\mathrm{Ia}}^{\mathrm{false}}=1\right)$, and only change the weighting factor to $W_{\mathrm{Ia}}^{\mathrm{false}}=5$ in the FoM. Our cut at $\chi_{r}^{2}=1.2$ is denoted by the vertical black line.

(A color version of this figure is available in the online journal.)

curves cross. Thus we define a PSNID-classified SNe Ia to be an object with $P_{\mathrm{Ia}}>P_{\mathrm{Ibc}}, P_{\mathrm{Ia}}>P_{\mathrm{II}}$, and $\chi_{r}^{2} \leqslant 1.2$.

The value of our $\chi_{r}^{2}$ cut differs significantly from that in $\mathrm{S} 11$, which can be seen in their Figure 10 to be located at a broad maximum of $\chi_{r}^{2} \simeq 1.8$. As noted in Appendix A, the version of PSNID used herein differs from that of S11 (which had larger model uncertainties), resulting in the optimal $\chi_{r}^{2}$ cut being smaller in this work.

\subsubsection{SALT2 Criteria}

Despite our optimization of the PSNID classification criteria, over $25 \%$ of our photometric SN Ia sample remains non-Ia SNe (see Figure 5 and Table 1). The purity of this sample is unsuitable for the cosmological analysis discussed in Section 4, which is the goal of this paper. We thus run the SALT2 ${ }^{33}$ light-curve fitter (described in detail in Appendix B) on all of our $\mathrm{SNe}$ (simulated and data) to obtain the best-fit light-curve parameters, and explore the effects of applying additional selection criteria to these parameters to further differentiate $\mathrm{SNe}$ Ia from non-Ia SNe.

In Figure 6 we show the distribution of the measured SALT2 parameters color $(c)$ and "shape" $\left(X_{1}\right)$ for all SNe remaining in our photometric SN Ia sample. By definition, SNe Ia form a welldefined cluster of points centered on zero in this parameter space, while non-Ia SNe are more scattered. In Lampeitl et al. (2010a) independent limits were placed on $X_{1}$ and $c$, but it is clear that an ellipsoidal cut (similar to the circular cut of Bazin et al. 2011) would yield a higher SN Ia purity for a given efficiency. We determine the optimal lengths of the semimajor $\left(a_{x_{1}}\right)$ and semiminor $\left(a_{c}\right)$ axes of this ellipse in Figure 7, which shows

33 We use only SALT2 throughout this paper. As we are interested in the utility of photometric samples of SNe Ia for cosmology, rather than presenting definitive cosmological constraints, we forego a detailed comparison of light-curve fitting algorithms. 


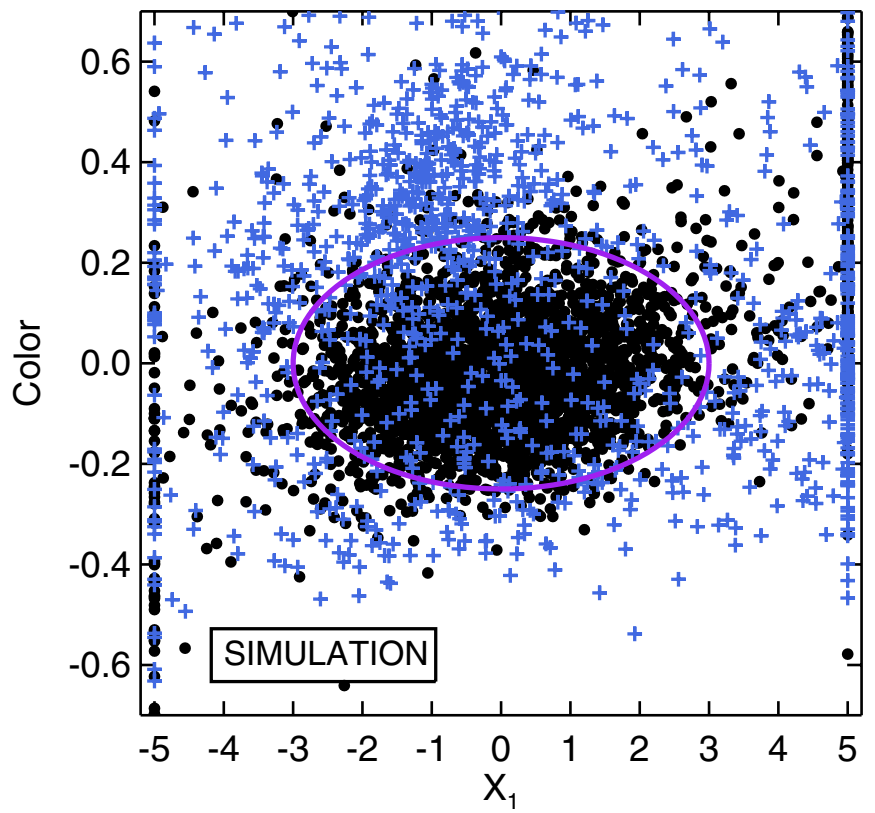

Figure 6. $X_{1}$ and color (c) distributions for the simulated $\mathrm{SNe}$ remaining in our photometric sample after PSNID cuts with SNe Ia as black points and non-Ia $\mathrm{SNe}$ as blue cross symbols. The purple ellipse defines the area we keep for the photometrically classified sample of SNe Ia. The cutoff at -5 and 5 in $X_{1}$ are hard limits set by SALT2.

(A color version of this figure is available in the online journal.)

the efficiency, purity, and FoM as a function of the ellipsoidal parameters. For an ellipse centered at $\left(x_{1}, c\right)=(0,0)$, the FoM shows a clear peak at $a_{x_{1}}=3$ and $a_{c}=0.25$. We use this ellipse, shown in Figure 6, to further distinguish SNe Ia from non-Ia SNe. As can be seen in Table 1, this procedure removes $\sim 70 \%$ of our contaminating $\mathrm{SNe}$ at the expense of rejecting $\sim 20 \%$ of the SNe Ia. We investigated allowing the center of the ellipse to vary, but found this did not significantly improve the purity or efficiency.

\subsubsection{Color-Magnitude Cut}

Bazin et al. (2011) recently showed that a cut in observed color-magnitude space significantly improves SN Ia sample purity by removing core-collapse $\mathrm{SNe}$ contaminants. We investigated the effects of such a color-magnitude cut on our simulations using the gri peak magnitudes from the bestfit SALT2 model for each SN. We found the most effective color-magnitude cut is in the $g-r$ versus $g$-band model magnitude plane. The optimal orientation of this color-magnitude cut is described by $g-r<0.3 \times(g-21.2)$, and is shown in Figure 8. This diagnostic cleanly removes a population of non-Ia SNe in the simulations which are too "blue" at the given magnitude to be SNe Ia. We found that the application of additional color-magnitude constraints, e.g., using different filters, did not produce a significant reduction of our contamination beyond the first color-magnitude cut.

\subsubsection{Overall Contamination in the Simulations}

In Table 1 we break down the effects of our selection criteria on our SN sample. The first row of Table 1 lists the number of $\mathrm{SNe}$ of each type that are included in the simulation; successive rows contain the number of SNe that remain in our SN Ia sample after each of the criteria (as detailed in the previous sections) are applied. We provide the contamination $(1-$ purity, assuming
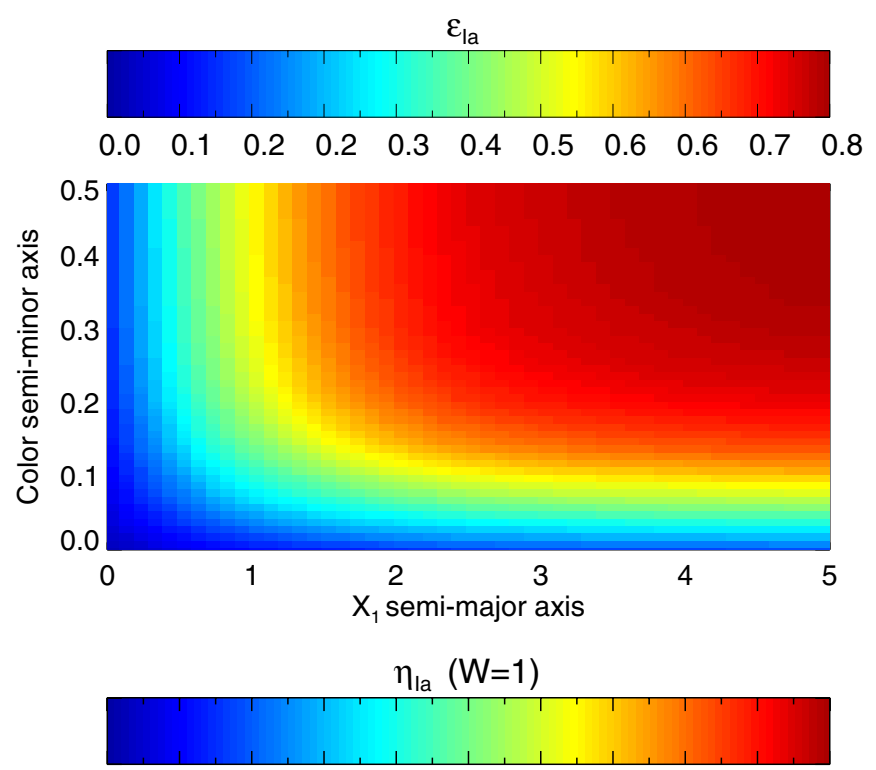

$\begin{array}{lllllllllll}0.80 & 0.82 & 0.84 & 0.86 & 0.88 & 0.90 & 0.92 & 0.94 & 0.96 & 0.98 & 1.00\end{array}$

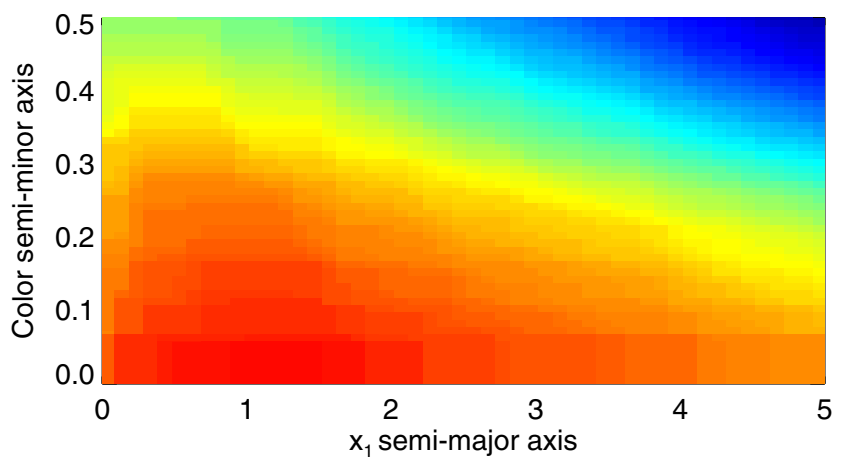

FoM $(\mathrm{W}=5)$

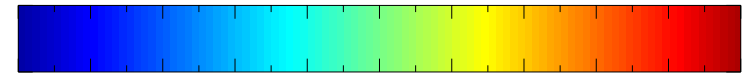

$\begin{array}{lllllllllll}0.10 & 0.15 & 0.20 & 0.25 & 0.30 & 0.35 & 0.40 & 0.45 & 0.50 & 0.55 & 0.60\end{array}$

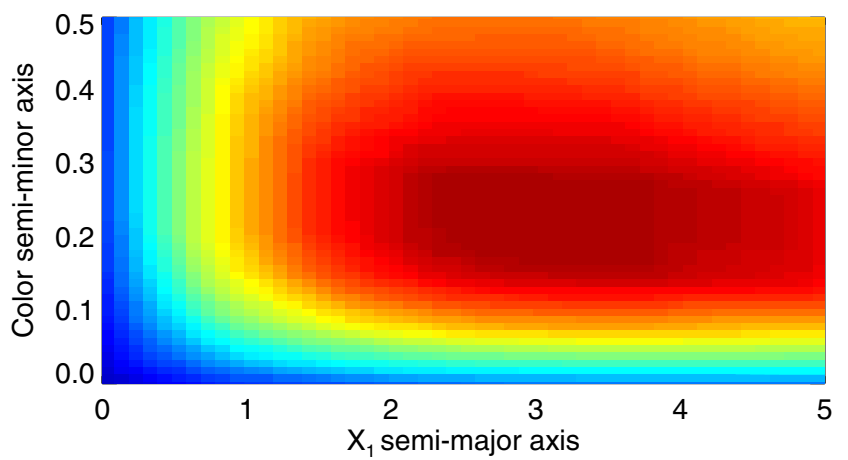

Figure 7. Efficiency (top), purity (middle), and FoM (bottom) plots from SN simulations, for changing the semimajor axis $\left(a_{X_{1}}\right)$ and semiminor axis $\left(a_{c}\right)$ of the ellipse in Figure 6.

(A color version of this figure is available in the online journal.)

$W_{\mathrm{Ia}}^{\text {false }}=1$ ), efficiency, and FoM (assuming $W_{\mathrm{Ia}}^{\text {false }}=5$ ) after the application of each selection cut. These quantities are defined using the number of SNe Ia that pass our light-curve quality cuts as the total number of SNe Ia, i.e., $N_{\mathrm{Ia}}^{\mathrm{CUT}}$ in Equation (1).

Application of both the PSNID and SALT2 criteria significantly improves the purity of our sample, resulting in an SN Ia sample with purity $>90 \%$ and efficiency $>70 \%$. The FoM 


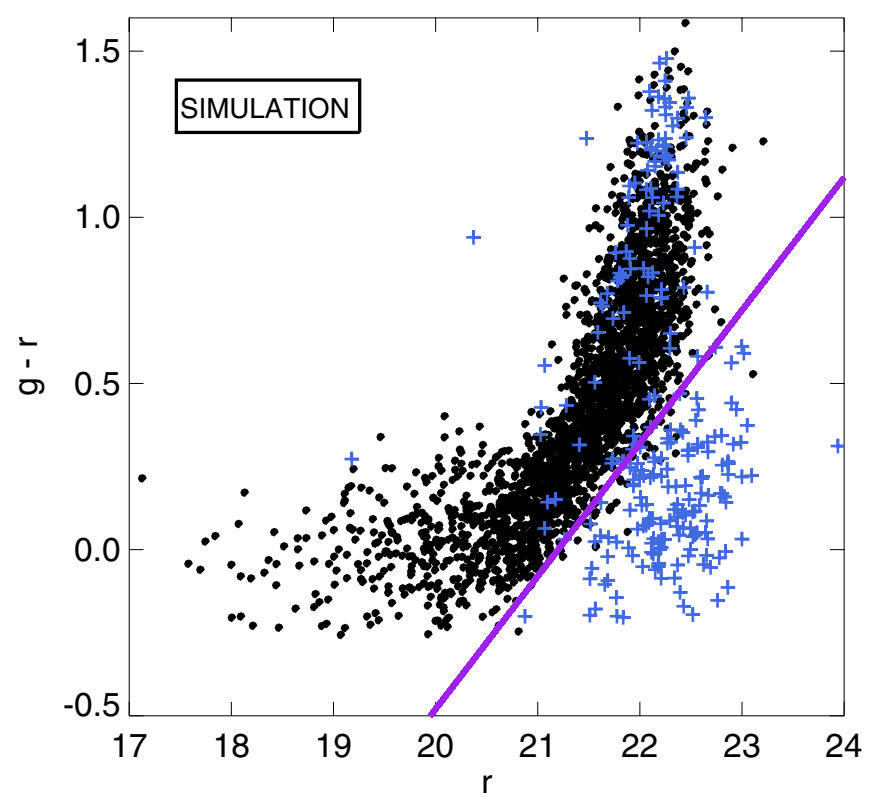

Figure 8. $\mathrm{SN} g-r$ color as a function of peak SN $g$-band magnitude derived from the best-fit SALT2 model to our simulations. The black dots are SNe Ia and the blue cross symbols are non-Ia SNe. The purple line is our color-magnitude cut that best separates non-Ia SNe contaminants from our sample.

(A color version of this figure is available in the online journal.)

has also increased significantly with these cuts, reflecting the weighting we have applied to purity. Interestingly, the inclusion of the color-magnitude constraints (Figure 8) significantly improves our purity with almost no effect on our efficiency.

Figure 9 is the Hubble diagram for our simulated photometrically classified SN Ia sample. The plotted errors are a combination of the uncertainties on the SALT2 light-curve parameters, the redshift and an assumed intrinsic dispersion of $\sigma_{\text {int }}=0.12 \mathrm{mag}$. The SALT2 SN Ia parameters are fixed to the input simulation values of $\alpha=0.11$ and $\beta=3.2$.

We show in blue in Figure 9 the 106 misclassified SNe that have passed all of our selection cuts. This final simulated photometric SN Ia sample has a contamination of $3.9 \%$ and an efficiency of $70.8 \%$. Our purity is higher than that for any of the participating methods in the "Photometric SN Classifier Challenge" (K10b), although it is necessary to note that we have explicitly placed a higher priority on purity than was the stated goal in K10b, and their analyses used a DES-like set of simulations that extend to higher redshifts than in our sample. As expected, the source of our contamination is primarily $\mathrm{SNe} \mathrm{Ib} / \mathrm{c}$, as their light curves most closely resemble those of an $\mathrm{SNe}$ Ia.

Finally, in Figure 10, we show the expected purity and efficiency of our sample (based on simulations) as a function of redshift. Within the errors, we see no significant redshift dependence in our purity, but we do witness a significant fall in our efficiency at higher redshifts. This is expected, as we have prioritized purity over efficiency for our cosmological study. Larger simulations (see Section 7.2) are required to probe more subtle effects with redshift.

\subsection{Application of Selection Method to the Data}

We now apply our analysis and the selection criteria defined using our SN simulations to our SDSS-II BOSS SN sample. In Table 2, we provide a breakdown of the number of SNe classified at each stage of our selection (as discussed in Section 3.1). The

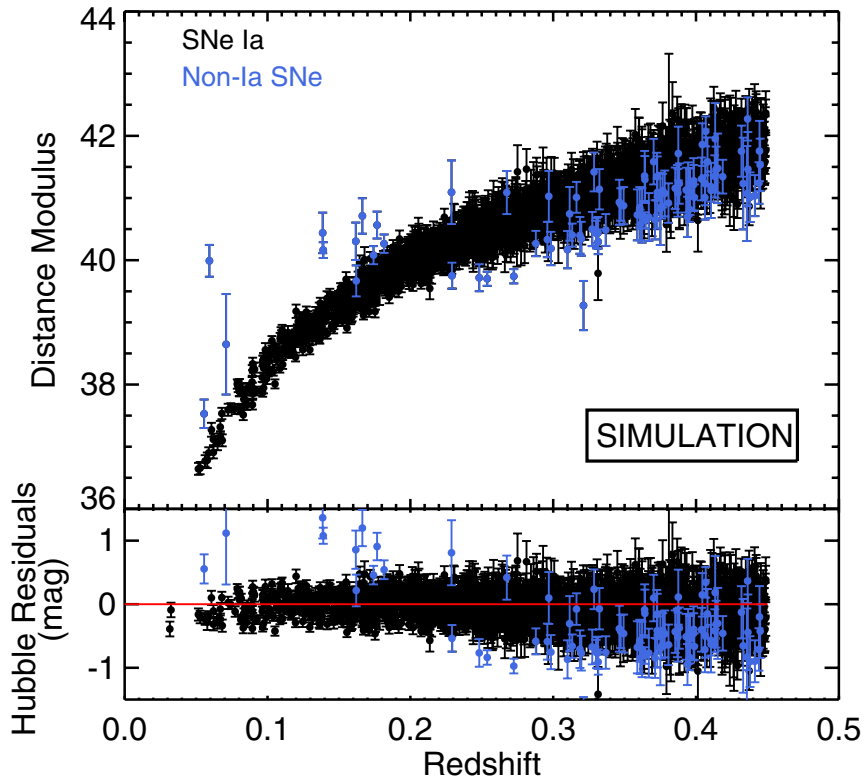

Figure 9. Hubble diagram of our simulated SNe Ia that pass all of our selection criteria. This includes 106 non-Ia SNe that have been misclassified (blue symbols) and 2644 correctly classified SNe Ia (black symbols). The redshift limit of $z=0.45$ is artificial and set by the original limit in the public SNANA simulations. The plotted errors are a combination of the uncertainties on the SALT2 light-curve parameters and the redshift. The SALT2 SN Ia parameters have been fixed to the input simulation values of $\alpha=0.11, \beta=3.2$, and $M=29.8$. The bottom panel shows the Hubble residuals assuming the input cosmology.

(A color version of this figure is available in the online journal.)

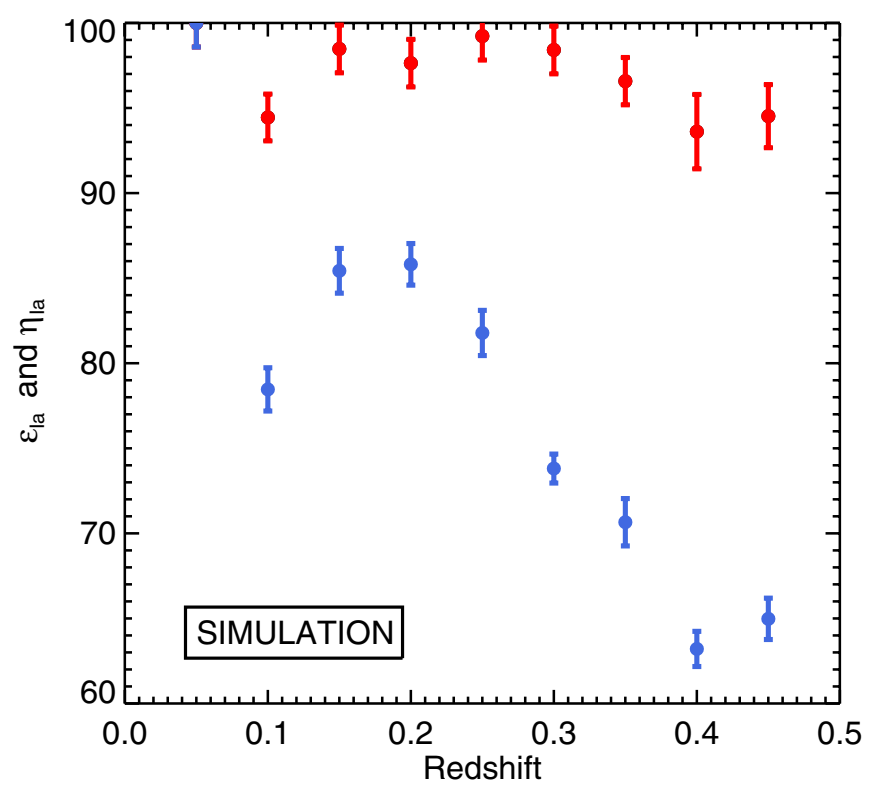

Figure 10. Simulated efficiency (blue) and purity (red) for our final photometrically classified sample. The error bars are determined from the propagation of errors using Equations (1) and (2).

(A color version of this figure is available in the online journal.)

two PSNID-based criteria remove $\approx 45 \%$ of the sample that remain after the data quality cuts we applied (comparable to the $48 \%$ seen in our simulations), resulting in 1443 objects in our SN Ia sample at this stage of analysis.

We use SALT2 (Appendix A), applied to the SDSS SMP griz light-curve data, to measure the light-curve parameters and distance moduli for all $3500(2382+941+177)$ SN candidates 
Table 2

The Cumulative Effect of Applying each Selection Criteria to Our Data, Leading to a Final Sample of Photometrically Classified SNe Ia (752)

\begin{tabular}{lcccc}
\hline \hline Selection Criteria & Removed SNe & Kept SNe & Spec SNe Ia & Spec Non-Ia SNe \\
\hline Accurate BOSS redshifts & - & 3500 & 329 & 59 \\
Light-curve quality & 874 & 2626 & 249 & 24 \\
$P_{\text {Ia }}>P_{\text {non-Ia }}$ (PSNID) & 579 & 2047 & 247 & 6 \\
$\chi_{r}^{2}<1.2$ (PSNID) & 604 & 1443 & 239 & 2 \\
$X_{1}$ and $c$ cut (SALT2) & 634 & 809 & 209 & 0 \\
Color-magnitude criteria (SALT2) & 54 & 755 & 209 & 0 \\
Correct host galaxy & 3 & 752 & 208 & 0 \\
\hline
\end{tabular}

Notes. The right-hand column shows the effect of these criteria on known (spectroscopically confirmed) SNe Ia in our sample.

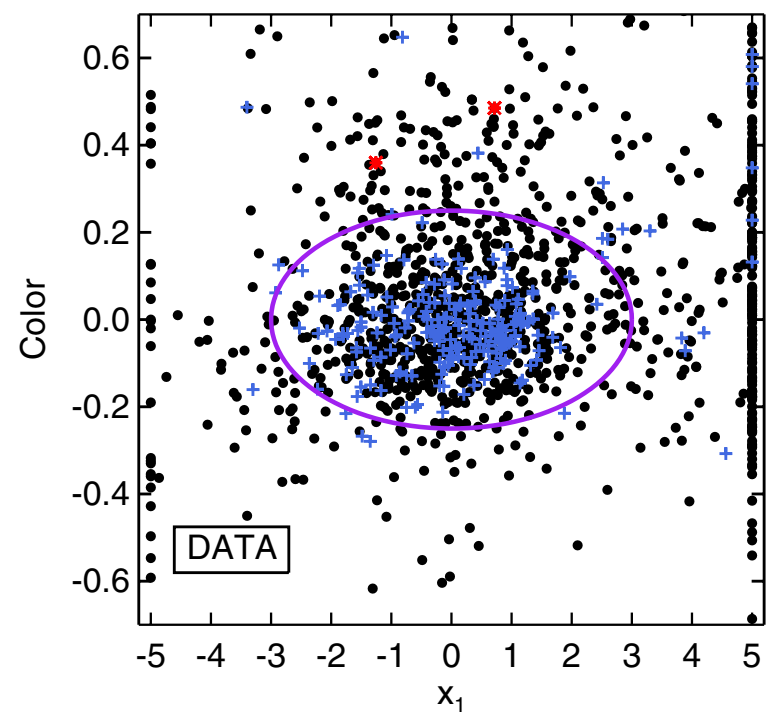

Figure 11. $X_{1}$ and $c$ distributions for PSNID-classified SNe Ia from our SDSS-II SN candidate samples. The blue (red) cross symbols denote the subset of these candidates that have been spectroscopically confirmed as SNe Ia (non-Ia SNe). The purple ellipse is our SNe Ia boundary taken from Figure 6.

(A color version of this figure is available in the online journal.)

with host-galaxy redshifts (see Section 2.3). Figure 11 shows the distribution of our PSNID-classified SN Ia sample in $X_{1}$-color space and the ellipsoidal criteria we derived from simulations. This cut removes $634 \mathrm{SN}$ candidates (44\%) from our photometric sample. This is a higher percentage than predicted by simulations in Section 3.1 (39\%), which could be attributed to the fact that non-SN contaminating sources (e.g., quasars, which are known to be in our BOSS target list) are not modeled in our simulations. Many of the discarded SN candidates have poorly fit templates, with $X_{1}= \pm 5$, where SALT2 is driven to the extremes of its self-imposed (i.e., hardcoded) $X_{1}$ range. This constraint also removes many "red" objects with large $c$ values, which may include highly reddened $\mathrm{SNe}$ Ia. However, we would prefer to exclude these red SNe Ia from our cosmology analysis, as Foley et al. (2010a) showed they potentially bias cosmological constraints. Our SALT2 criteria leave us with 809 photometrically classified SNe Ia candidates.

In Figure 12 we show the application of the color-magnitude cut in the $g-r$ versus $g$-band model magnitude plane, which removes a sample of "blue" SN candidates. Both in the data and the simulations this cut removes $\approx 7 \%$ of the sample that passed the SALT2 cut, leaving a photometrically classified sample of $755 \mathrm{SNe}$ Ia candidates. The agreement between the simulations and data is reassuring, and it should be noted that

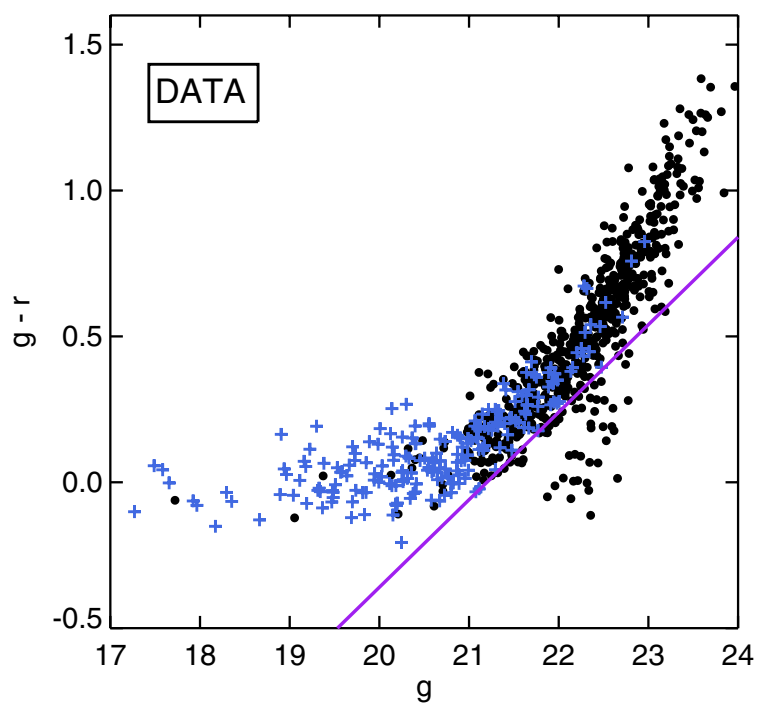

Figure 12. $g-r$ color as a function of $g$-band magnitude for our SDSS-II data. The blue cross symbols denote the subset of these candidates that have spectroscopic confirmation as SNe Ia. The location of the purple line is our SN Ia boundary taken from Figure 8 .

(A color version of this figure is available in the online journal.)

these simulations were made prior to the construction of our BOSS SDSS SN sample, so there has been no fine-tuning of the simulations to match our BOSS sample.

A potential source of error that is not included in our simulations is the misidentification of the host galaxy of our SN candidates, as this can result in an incorrect classification due to an incorrect redshift prior. We have identified three cases in our sample where we have evidence, described in Appendix C, that either the galaxy observed by our BOSS program is not associated with the $\mathrm{SN}$ to which it was assigned, or the derived BOSS redshift is erroneous. We remove these three $\mathrm{SN}$ candidates from our sample.

Our final sample contains 752 photometrically classified SNe Ia, of which 208 have been spectroscopically confirmed as SNe Ia as part of the original SDSS-II SN Survey (Table 2).

\subsection{Tests of Our Photometric Sample}

We present here a basic examination of our final sample of 752 photometrically classified SNe Ia before embarking on a cosmological analysis (Section 6). First, we study the effect of our selection criteria on the subset of existing spectroscopically confirmed SNe Ia in our sample. Unlike S11, we have not used this subset of known SNe to refine our selection criteria as there are concerns about potential bias in this sample (see Section 2.1). As can be seen in Table 2, we start with 329 


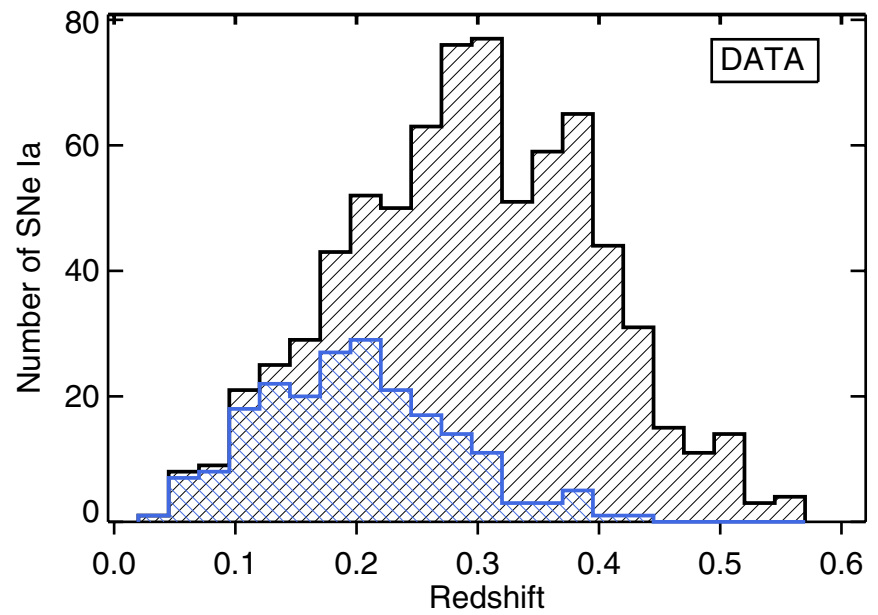

Figure 13. Redshift distribution of our 752 photometrically classified SNe Ia (black), and the subset of 208 that have spectroscopic classification (blue).

(A color version of this figure is available in the online journal.)

spectroscopically confirmed SNe Ia that have had host-galaxy redshifts obtained by BOSS or SDSS. Of these, $249 \mathrm{SNe}$ Ia passed our data-quality criteria applied in Section 3.1.3. After applying all of our additional selection criteria we are left with 208 spectroscopically confirmed SNe Ia, resulting in an efficiency of $84 \%(208 / 249)$ in classifying our SDSS-II spectroscopic SN Ia sample.

This efficiency is higher than the predicted value from our simulations (71.6\%; see Table 1$)$. This difference can mainly be attributed to the spectroscopic sample probing a lower redshift range than the photometric sample. In Figure 13 we show the redshift distribution for our full sample of photometrically classified SNe Ia (black) compared to the subsample of spectroscopically confirmed SNe Ia (blue). While the spectroscopic sample peaks at $z \sim 0.2$ and drops to zero by $z>0.4$, the photometrically classified $\mathrm{SNe}$ Ia extend out to $z \simeq 0.55$, with a median redshift of $z=0.30$. This explanation is checked by studying the "spectroscopically confirmed" subset of SNe Ia provided as part of the public SNANA simulations discussed in Section 3.1.1. The efficiency of photometrically classifying this simulated spectroscopic SNe Ia subset is $83.2 \%$, in reassuring agreement with the $84 \%$ efficiency we find for the spectroscopic SNe Ia subset in our data.

We reiterate that we do not include in our final sample any of the spectroscopically confirmed SNe Ia that were removed during our photometric-classification procedure. Our intention is to construct a sample of $\mathrm{SNe}$ Ia based purely on their photometric properties and host-galaxy redshifts, and examine the cosmological constraining power of such a sample. This procedure mimics the challenges facing the next generation of SN surveys.

All spectroscopically confirmed non-Ia $\mathrm{SNe}$ in the original SDSS-II SN sample were removed by our selection criteria, resulting in $100 \%$ purity for the spectroscopic subset of our photometric sample. Of the 59 known non-Ia SNe with hostgalaxy redshifts obtained by BOSS, only two were misclassified as SNe Ia by PSNID, and both were subsequently removed by the $X_{1}-c$ criteria. There are two factors that lend themselves to this subset achieving higher purity than would otherwise be expected from our simulations. First, the SDSS-II spectroscopic program was intentionally biased against targeting non-Ia $\mathrm{SNe}$; in our BOSS sample there is only one spectroscopically confirmed

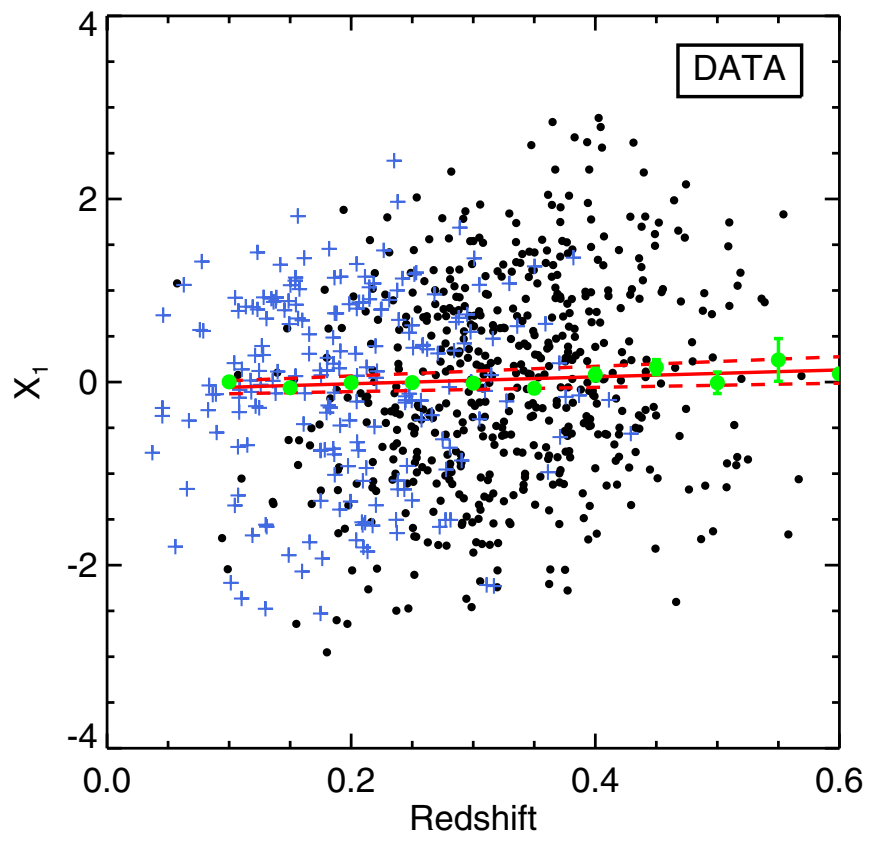

Figure 14. SALT2 $X_{1}$ parameter as a function of redshift for the photometrically classified SNe Ia (black dots) and the subset of spectroscopically confirmed SNe Ia (blue crosses). The green points show the mean $X_{1}$ (and the error on the mean) in bins of redshift. The red solid line is the best-fit linear relation to the average $X_{1}$, and the red dashed lines are the error on the fit.

(A color version of this figure is available in the online journal.)

non-Ia SN for every six SNe Ia, whereas nearly $60 \%$ of the $\mathrm{SNe}$ in our simulations are non-Ia. Second, PSNID was tested, and optimized, on the SDSS-II spectroscopic SNe, so its efficiency at identifying non-Ia $\mathrm{SNe}$ from this sample is unsurprising.

In Figure 14, we show the SALT $X_{1}$ values as a function of redshift for the photometrically classified SNe Ia sample. We also show the mean $X_{1}$ in redshift bins and the best-fit linear relation to the binned data. We see no change in the distribution of $X_{1}$ values with redshift, as the best-fit slope is consistent with zero within the errors on the fit. In Figure 15, we show the SALT $c$ (color) values as a function of redshift, again with their mean values, binned by redshift, and the best-fit linear relation. A correlation is evident in this case, with higher redshift $\mathrm{SNe}$ skewed toward bluer (negative) colors. However, this trend is driven by SNe at $z>0.4$, and if we limit the sample to redshifts below this value we find the best-fit slope is consistent with zero within errors. We may be seeing evidence of a color-dependent Malquist bias, i.e., bluer SNe Ia are brighter and thus easier to detect at higher redshifts (more so than $\mathrm{SNe}$ with higher $X_{1}$ values).

In Figure 16 we present two Hubble diagrams for our photometrically classified sample of SNe Ia. On the left is the sample before our color-magnitude cut is applied, while on the right we show our sample after making this cut. This criterion removes $54 \mathrm{SNe}$ Ia candidates, which are clearly offset (too faint) from the main Hubble sequence and thus likely to be nonIa $\mathrm{SNe}$ (as seen in our simulations). These removed candidates are also clustered around $z \approx 0.2$, again consistent with our simulations.

There are still 10 possible outliers, around $z \approx 0.2$, to the main Hubble diagram in Figure 16 (right-hand side). We have studied the photometric data for these Hubble residual (HR) outliers individually (including visually inspecting the images) and can find no obvious reasons to remove them from our sample. These 


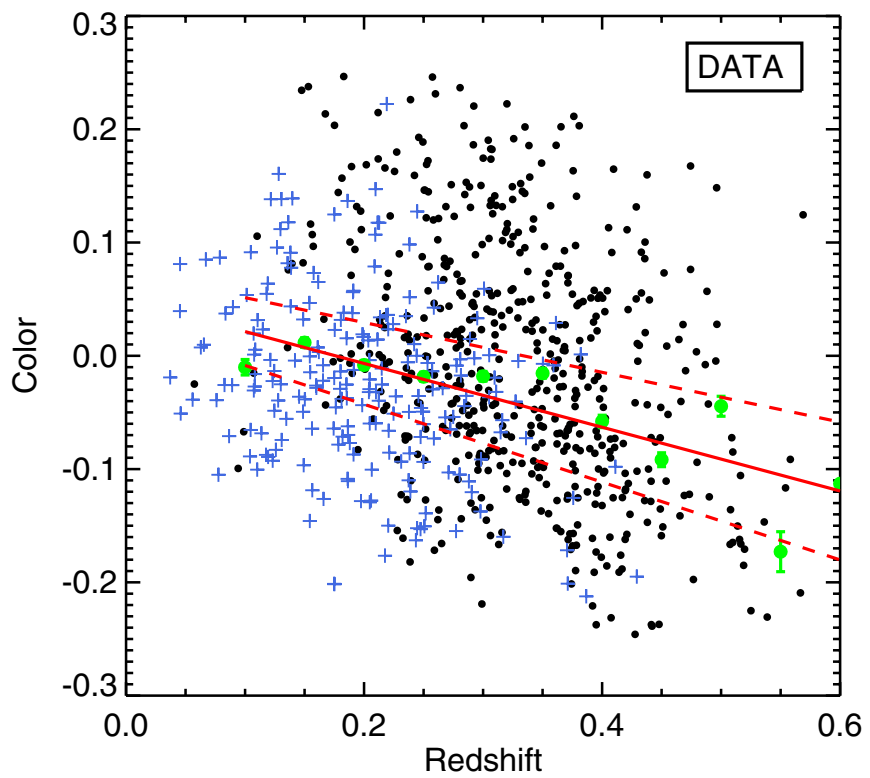

Figure 15. SALT2 color parameter as a function of redshift for the photometrically classified SNe Ia (black dots) and the subset of spectroscopically confirmed-SNe Ia (blue crosses). The green points show the mean color (and the error on the mean) in bins of redshift. The solid red line is the best-fit linear relation to the average color, and the dashed red lines are the error on the fit.

(A color version of this figure is available in the online journal.)

outliers could be Type Ibc SNe, which possess similar colors and light-curve shapes in the SDSS filters at these redshifts, or possibly odd SNe Ia such as PTF10ops (Maguire et al. 2011) and SN 2006bt (Foley et al. 2010b). However, this small amount of contamination does not bias our cosmological results, as shown in Section 6.1, where we apply at $3 \sigma$ clipping to the Hubble diagram and find consistent cosmological results.

\section{SOURCES OF BIAS}

Before we undergo a detailed cosmological analysis of our sample (Section 6), we investigate possible sources of bias in our photometrically classified sample.

In Figure 17 we show the residuals about the Hubble diagram from our simulations (Section 3.1); the HR is the difference between the observed and input distance modulus for each SN Ia. We have subtracted the true distance modulus, based on the input cosmology, from the measured distance modulus for each $\mathrm{SN}$ in the simulation, and binned the results in redshift. The left panels of Figure 17 show the SNe Ia photometrically classified using the methodology outlined in Section 3, while the right-hand panels show residuals for the true SNe Ia in the simulations. Each row demonstrates the effect of adding one of our selection criteria, described in Section 3.1. In the absence of any systematic bias, we would expect these residuals to be scattered about zero (blue line).

There are two sources of bias whose effects are easily seen in Figure 17. First, after making only the data-quality and PSNID cuts, our SN sample (second row, left panel) shows a strong bias toward positive HRs (underluminous objects) at $z<0.2$. We have shown in Table 1 that this sample has high contamination, i.e., at $z \leqslant 0.25$ non-Ia $\mathrm{SNe}$, though fainter than $\mathrm{SNe} \mathrm{Ia}$, are still bright enough to be detected as SN candidates in the SDSS SN Survey. At higher redshifts core-collapse SNe are too faint to be observed, and thus their contamination is naturally curtailed. This bias at low $-z$, due to contamination, is effectively eliminated once the $X_{1}$-color cut is introduced. After the final color-magnitude cut (bottom panel) the HRs are consistent with zero bias at low redshifts.

At high redshifts $(z>0.25)$ the HRs in all panels of Figure 17 are biased low; i.e., $\mathrm{SNe}$ are, on average, brighter than expected given the input cosmology. This selection effect is a combination of the classic Malmquist bias (Malmquist 1936;

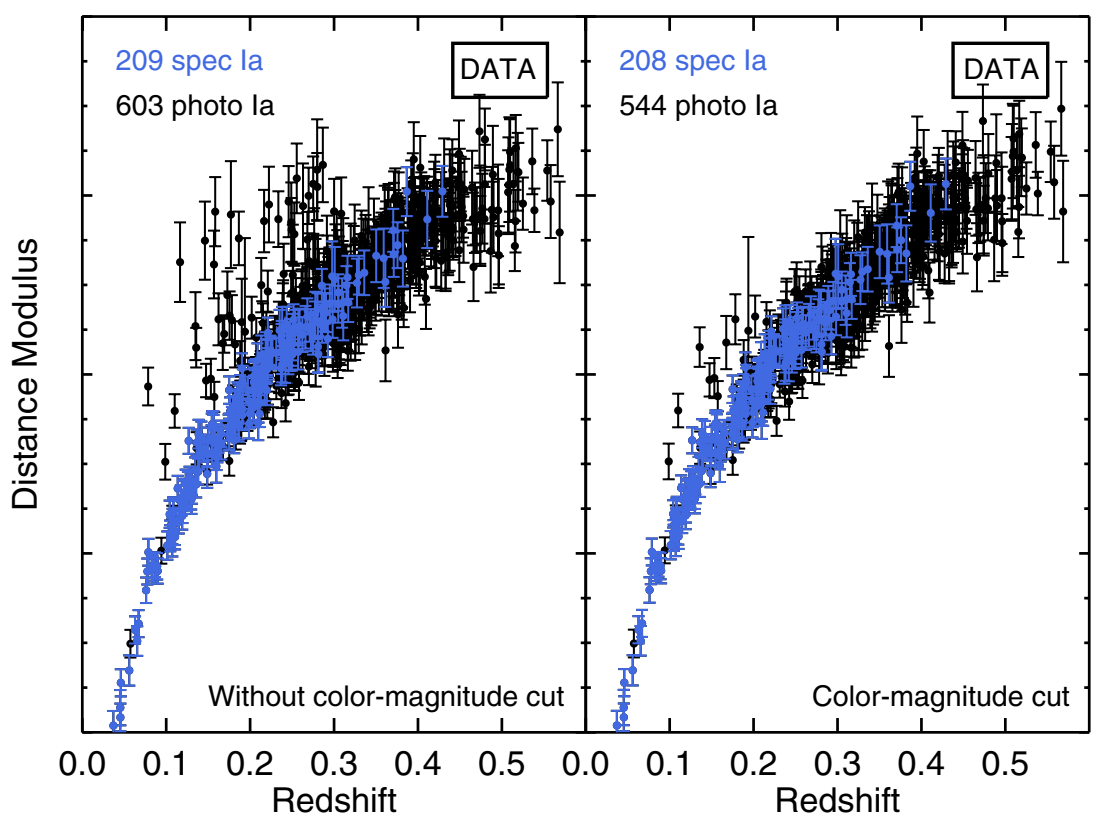

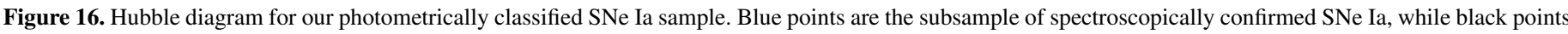

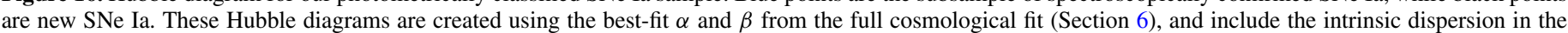

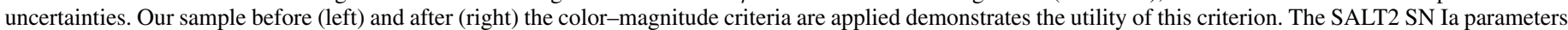

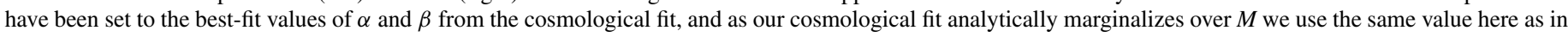
the simulations $(M=29.8)$.

(A color version of this figure is available in the online journal.) 


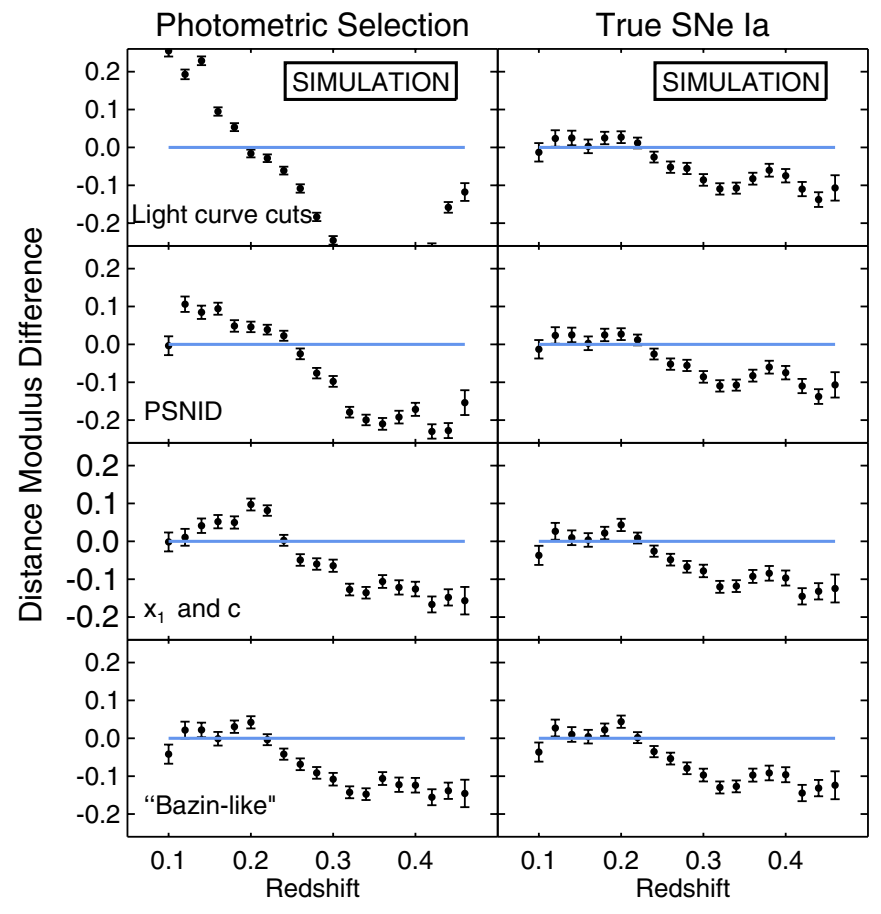

Figure 17. Weighted mean and uncertainty for Hubble residuals in our simulations in bins of $\Delta z=0.02$. The left-hand panels show the photometrically classified SNe Ia, while the right-hand panels are for true SNe Ia. Each row shows the cumulative effect of applying the various selection criteria discussed in Section 3.1.

(A color version of this figure is available in the online journal.)

Wood-Vasey et al. 2007) and other SALT2-related selection effects. We have only analyzed objects that would be classified as SN candidates in the SDSS-II SN Survey (survey pipeline cuts and post-survey cuts are applied within the simulations), so underluminous objects at high- $z$ are never output from the simulations. Additionally, though we place no implicit $\mathrm{S} / \mathrm{N}$ cut on our data, low $\mathrm{S} / \mathrm{N}$ SNe that are poorly fit by SALT2 are more likely to fail the $X_{1}-c$ cut, and our original data-quality cut, and are thus preferentially excluded (see Section 5.1).

For clarity, we show in Figure 18 the difference between the recovered distance moduli for the photometrically classified $\mathrm{SNe}$ Ia and the true SNe Ia as a function of redshift, i.e., the bottom left-hand panel in Figure 17 minus the bottom right panel in Figure 17. This removes the Malmquist bias effect, allowing us to see the residual contamination bias as a function of redshift. As one would infer from Figure 10, the redshift-dependent bias of our HRs due to contamination is seen in Figure 18 to be a small effect. We find, using the Akaike information criterion (Akaike 1974) to compare the two models, that a linear fit is favored over a redshift independent model, with a slope of -0.021 best describing the linear bias in the measured redshift range. This potential redshift dependence deviates from null by only $-0.01 \mathrm{mag}$ out to $z=0.5$. Thus the effect of the contamination is subdominant to the Malmquist bias, which has modeled uncertainties that are larger than the possible redshift dependence of the contamination bias.

The combination of these selection effects (the classical Malmquist bias and SALT2 effects) is by far the biggest source of bias affecting photometric SN sample if left uncorrected, which is discussed in detail in Section 4.1. However, we note that such effects, especially the classical Malmquist bias, are also present in spectroscopic samples where they are more difficult to correct for, as this bias will depend on the details

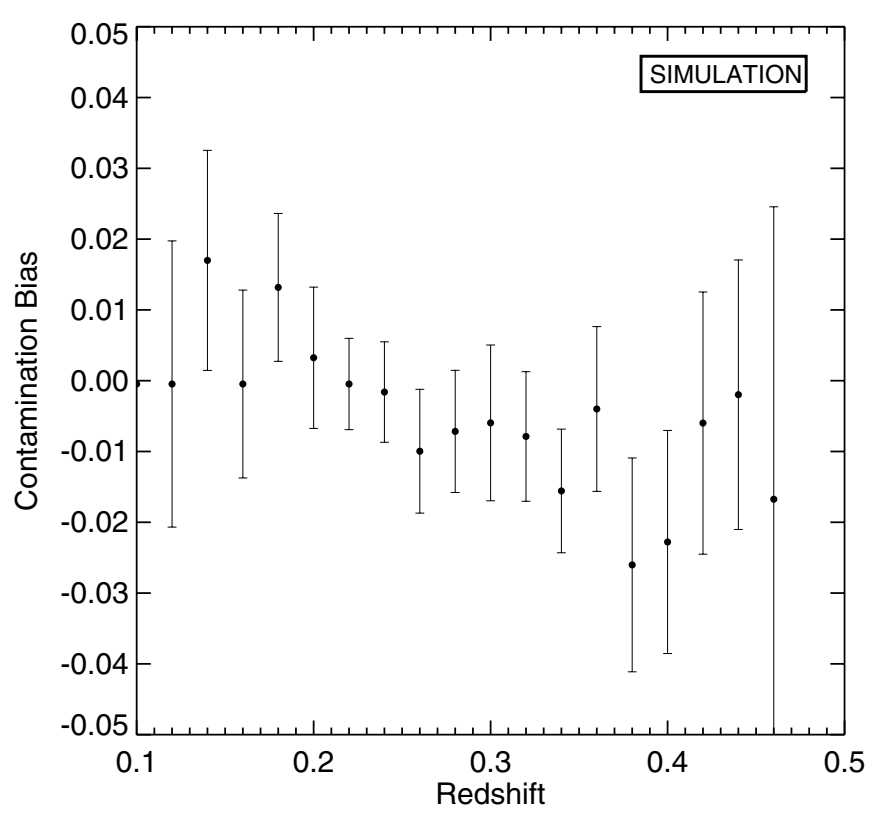

Figure 18. Difference between the weighted mean of the Hubble residuals from the photometrically classified SNe Ia and the true SNe Ia, i.e., the difference between the bottom two panels in Figure 17. The error bars account for the correlations between the photometrically classified SNe Ia and the true SNe Ia.

of the spectroscopic program (Sako et al. 2008; Kessler et al. 2009a).

\subsection{Selection Effects}

In this section we correct for the known selection bias at $z>0.25$ seen in Figure 17. This bias is a combination of the classical Malmquist bias and SALT2-related effects. For a magnitude limited SNe survey, the classical Malmquist bias results for a given $X_{1}$ and color in the preferential detection of $\mathrm{SNe}$ that appear brighter due fluctuations caused by Poisson noise and intrinsic scatter. We do not attempt to study these different effects separately in this paper and only provide a correction for their combined effect on the distance moduli of our photometric SNe Ia sample. For this reason, we will hereafter refer to this combined selection effect as just the "Malmquist bias," but ask the reader to recognize that it is a combination of magnitude (or $\mathrm{S} / \mathrm{N}$ ) effects, of which the classical Malmquist bias is likely the most important.

Before we present the details of our correction to the Malmquist bias, we first demonstrate the importance of this effect in our cosmological analysis. We compute the equation of state of dark energy $(w)$ for the entire sample of true SNe Ia that pass data-quality cuts (top right panel of Figure 17) using the publicly available software package CosmoMC (Lewis \& Bridle 2002), including priors on the cosmological parameters (we detail our further usage of CosmoMC in Section 6). We find the best-fit cosmology for this sample to be $w=-0.90 \pm 0.05$, which is inconsistent with the input cosmology of $w=-1$ (though only at the $2 \sigma$ level), and may indicate a bias. We stress that this sample is, by definition, $100 \%$ pure and $100 \%$ efficient, yet still produces biased cosmological parameters.

Therefore, to investigate the selection effects bias, we have undertaken a new set of SNANA simulations that span a wider range in redshift than those used in our analysis of the sample contamination (Section 3.1.1). We also increase the number of $\mathrm{SNe}$ in the simulation, as the Malmquist bias is small compared to the size of the error bars and requires sufficient $\mathrm{S} / \mathrm{N}$ to characterize it. In detail, we use SNANA (version v9_97; Kessler 


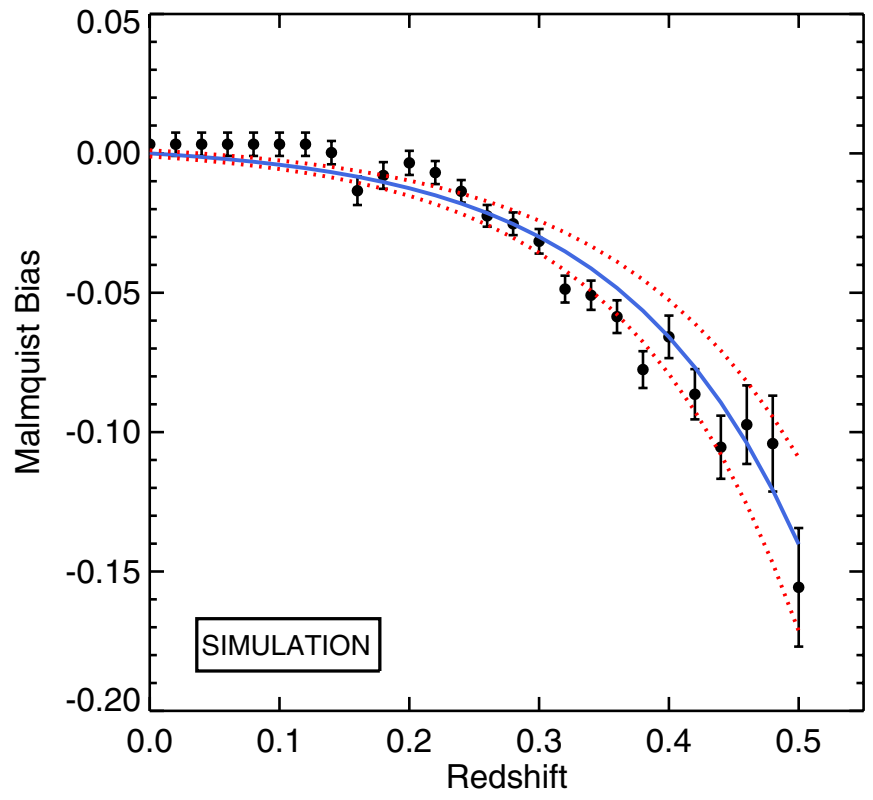

Figure 19. Difference between the observed $\left(\mu_{\mathrm{obs}}\right)$ and expected $\left(\mu_{\text {cosmo }}\right)$ distance modulus as a function of redshift for the simulations described in Section 4.1. We show the best-fit exponential function to these data (blue line) and the $1 \sigma$ errors on the fit (dashed red line).

(A color version of this figure is available in the online journal.)

et al. 2009a) to create $30,000 \mathrm{SNe}$ Ia over the redshift range $0.01 \leqslant z \leqslant 0.5$. For consistency, the SALT2 SN parameters and the assumed cosmology are the same as those used for the public SNANA simulations described in Section 3.1.1. As we are characterizing an effect for $\mathrm{SNe} I a$, we do not include core-collapse $\mathrm{SNe}$ in the simulation.

In Figure 19, we show the weighted mean difference between the "observed" $\left(\mu_{\text {obs }}\right)$ and true $\left(\mu_{\text {cosmo }}\right)$ distance modulus for our simulated $\mathrm{SNe}$ Ia after applying our photometric-classification criteria to the data. ${ }^{34}$ We use Equation (B1) to calculate $\mu_{\mathrm{obs}}$, fixing $\alpha$ and $\beta$ to the values used in the simulation. ${ }^{35}$ There is little bias out to $z \simeq 0.3$, but at higher redshifts a significant offset appears, growing with increasing redshift and becoming $\gtrsim 0.1$ magnitude overluminous.

We also show in Figure 19 the best-fit analytical function to the Malmquist bias, which is an exponential of the form

$$
\mu_{\mathrm{corr}}(z)=a e^{b z}+c,
$$

where $a=-0.004 \pm 0.001, b=7.26 \pm 0.31$, and $c=$ $0.004 \pm 0.006$. We include this Malmquist bias correction in our distance modulus calculation (Equation (B1)), resulting in a better estimate of the average distance modulus with redshift. This correction is based on simulations of the SDSS-II SN Survey, and thus is only valid for these data samples and selection criteria.

We note that the differences between the plot of $\Delta \mu$ in the bottom right panel of Figure 17 and that in Figure 19 are the

\footnotetext{
34 We apply all our data cuts to the simulated data except for the PSNID cuts, which require significant computational time for such a large suite of simulations. We have confirmed that excluding this cut does not affect our results using the public SNANA simulations since we are only interested in using true SNe Ia for the Malmquist bias correction. Only an additional $7 \%$ of $\mathrm{SNe}$ Ia in these simulations are removed by this cut. We have also demonstrated that the shape of the Malmquist bias is no different whether or not the PSNID cut is included.

35 Allowing $\alpha$ and $\beta$ to vary during the computation of $\mu$ does not significantly change our Malmquist bias correction, but does increase the statistical error.
}

reflection of more than simply an increase in statistics. In the public SNANA simulations of K10a an offset of 0.27 mag existed between the brightness of SNe Ia created in the simulations and those observed in Sullivan et al. (2011), resulting in fainter SNe Ia in the public simulations than are used in Figure 19. Thus the public simulations, while useful for our contamination analysis, should not be used for detailed cosmological calculations, although we have confirmed (with newer simulations) that Figures 4 and 5 remain unaffected by this offset.

The Malmquist bias we find is larger than has been previously reported by other surveys. This is primarily because we are pushing the SDSS-II SN survey to its limit of low $\mathrm{S} / \mathrm{N}$ observability; by $z=0.5$ only the very brightest $\mathrm{SNe}$ are observed. We note that the ESSENCE survey also found a significant Malmquist bias via their simulations, which they corrected for by adjusting the prior on the host-galaxy extinction $\left(A_{v}\right)$ as a function of redshift (Wood-Vasey et al. 2007). This is an alternative method to adding an average redshift-dependent correction to each SN Ia distance modulus, presented here.

\subsubsection{Testing the Malmquist Bias}

The above Malmquist bias correction is computed using a particular set of cosmological parameters. To determine whether or not this assumption biases our results, we have rerun our simulations with a set of widely varying input cosmologies, including $\left(\Omega_{\mathrm{m}}, \Omega_{\Lambda}, w\right)=(1,0,-1),(0.5,0.5,-1.5)$, and $(0.2,0.8,-0.8)$. We find that the parameters describing the Malmquist bias correction in Equation (4) do not change beyond their quoted statistical uncertainties.

The value for $\alpha$ that we have used in the simulations for deriving the Malmquist bias correction $(\alpha=0.11)$ is much lower than what we recover from the data $(\alpha=0.22$; Section 6.3$)$. We examine the effect this has on our results by determining the Malmquist bias correction from a new set of simulations that uses $\alpha=0.22$ in the input model. We find that the resulting Malmquist correction is consistent with what we previously derived; the $b$ parameter of Equation (4) is only changed by 0.14 (less than half the error), and the $a$ and $c$ parameters are unchanged.

To demonstrate the expected effect of the Malmquist correction on our photometrically classified SN Ia sample, we draw 10 subsamples of the same size and redshift distribution as our real data at random from photometrically classified $\mathrm{SNe}$ Ia in our simulations. We derive the best-fit cosmology for these samples both with and without the Malmquist bias correction, again using CosmoMC. For the uncorrected case we again find a best-fit value of $w=-0.87 \pm 0.03$, which is the weighted mean and uncertainty from the 10 randomly drawn samples. This result is significantly different from the input cosmology of $w=-1$, demonstrating the importance of the Malmquist correction, the application of which produces the expected best-fit value of $w=-1.00 \pm 0.03$.

\subsection{Host-galaxy Follow-up Bias}

An additional source of potential bias is due to the spectroscopic follow-up program of SN host galaxies. Our ancillary BOSS spectroscopy of likely SN Ia host galaxies (Section 2.2.1) had an apparent (fiber) magnitude limit of $r_{\text {fiber }}=21.2$. This should not cause an additional Malmquist bias, as the target selection is biased against fainter galaxies, not fainter $\mathrm{SNe}$. It is well known, though, that faint galaxies preferentially host only luminous (high $X_{1}$ ) SNe Ia (Hamuy et al. 1996b; Gallagher et al. 


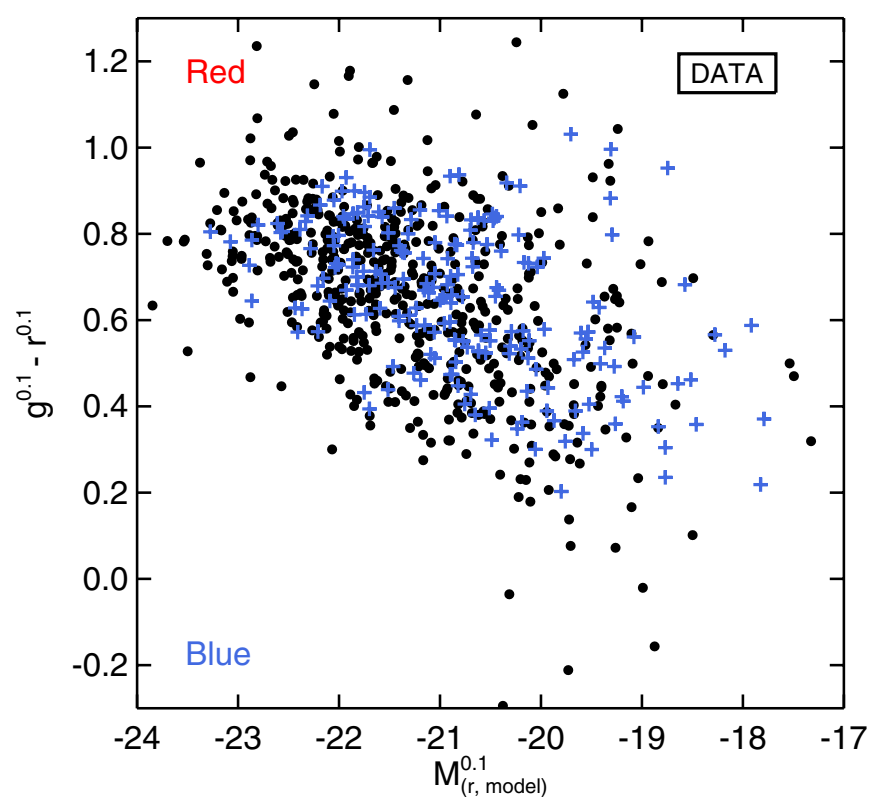

Figure 20. Host-galaxy $g-r$ color as a function of absolute $r$-band model magnitude (both quantities $k$-corrected to $z=0.1$ ) for the host galaxies of the photometrically classified SNe Ia (black dots) and the subsample of SNe Ia that have been spectroscopically confirmed (blue cross symbols).

(A color version of this figure is available in the online journal.)

2005), and thus the target selection would appear to favor detection of fainter SNe Ia. However, it has recently been shown that $\mathrm{SNe}$ in massive galaxies tend to be overluminous for their light-curve shape and color (Gallagher et al. 2008; Kelly et al. 2010; Sullivan et al. 2010; Lampeitl et al. 2010b). These effects combine with our host-galaxy magnitude limit in a complicated manner that is not captured in our simulations, but which merits further study in the future.

It is important to remember that despite these potential biases, photometric classification yields a less biased hostgalaxy sample than our spectroscopic sample. In Figure 20 we show the color-magnitude diagram for the host galaxies in our BOSS sample, with the subsample of spectroscopically classified $\mathrm{SNe}$ Ia shown in blue. For each galaxy, we plot the $g-r$ color and the absolute $r$-band model magnitude, both of which have been $k$-corrected using the standard SDSS software (Blanton \& Roweis 2007). As the default, we quote all absolute magnitudes and the $g-r$ color at $z=0.1$. The host galaxies of the spectroscopic subset are, on average, fainter than the whole population of BOSS host galaxies. A Kolmogorov-Smirnov test of the $k$-corrected model $r$-band absolute magnitude distributions of the two galaxy samples (spectroscopically confirmed $\mathrm{SN}$ hosts and photometrically confirmed SN hosts) confirms that the two distributions are not the same at a statistical significance of $99.9 \%$. The photometric sample includes intrinsically brighter host galaxies, which may be due to the increased volume sampled by the photometric sample as such luminous, massive galaxies are rare. It could also be a product of the SN spectroscopic follow-up avoiding the brightest hosts, as in these cases it is more difficult to separate the SN from the host-galaxy light. However, there does not appear to be a bias in the $g^{0.1}-r^{0.1}$ model colors, which is reassuring.

\section{HUBBLE DIAGRAM}

We show in Figure 21 the Hubble diagram for our final sample of 752 photometrically classified SNe Ia, derived from

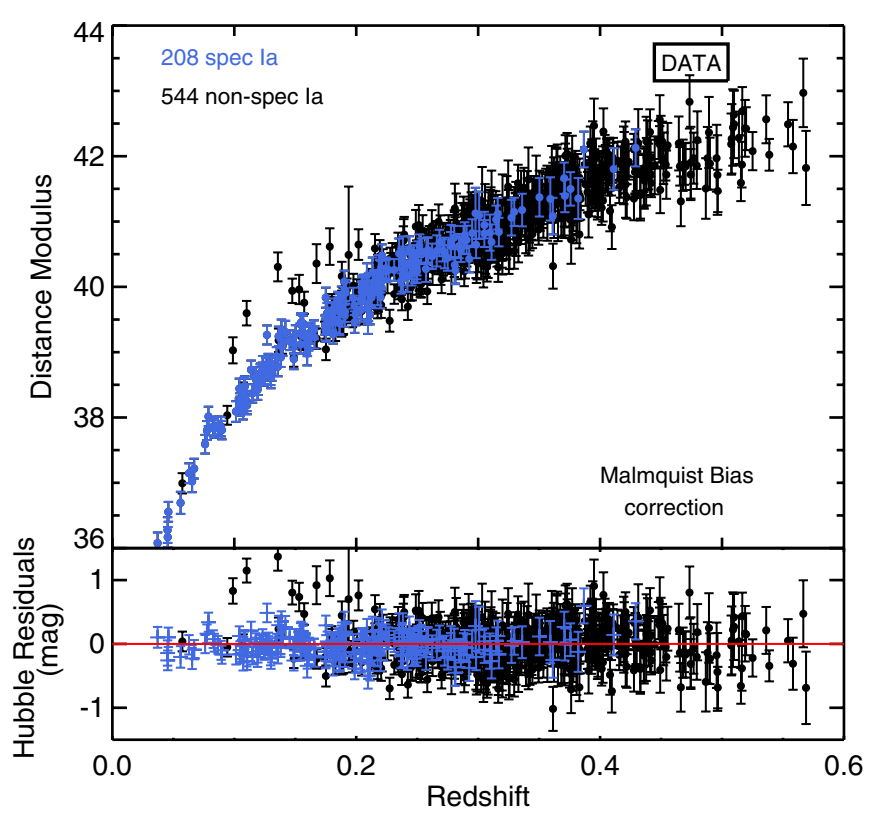

Figure 21. Hubble diagram of the photometrically classified SDSS-II SN Ia sample. We have corrected for the Malmquist bias as discussed in Section 4.1. We use the best-fit values of $\alpha$ and $\beta$ (see Section 6) and assumed the same $M$ as in the simulations $(M=29.8)$ when creating this Hubble diagram. The SN intrinsic dispersion has been included in the error bars shown. Blue points show the subsample of SNe Ia that have been spectroscopically confirmed as part of the SDSS-II SN Survey, while the black points only possess a photometric classification. The bottom panel shows the Hubble residuals of the data from the best-fit cosmology model (Section 6).

(A color version of this figure is available in the online journal.)

the SDSS-II SN Survey photometry and SDSS-III BOSS hostgalaxy spectroscopy (Section 3.2). In contrast to Figure 16, we have now applied our Malmquist bias correction to the Hubble diagram as derived in Section 4.1. We have not corrected our sample for host-galaxy mass correlations, as it is beyond the scope of this paper (see Section 7.1).

For comparison, we highlight in Figure 21 the subsample of $208 \mathrm{SNe}$ Ia in our photometric sample that have spectroscopic confirmation from the SDSS-II SN Survey (shown in blue), and label this subsample "spec Ia." Therefore, 544 of our photometrically classified SNe Ia are have no spectroscopic information at all, comprising $72.2 \%$ of the sample. We note that only 115 of these $544 \mathrm{SNe}$ Ia have been previously photometrically classified, using host-galaxy spectra from the SDSS-I/II surveys (S11; Hlozek et al. 2012). The data for all SNe Ia in our final sample can be found in Appendix E.

\subsection{Increased Scatter}

The bottom panel of Figure 21 appears to show an increase in the scatter of the HRs for the photometrically classified SNe Ia (black points) compared to the spectroscopically confirmed subsample (blue points). In Figure 22, we study this apparent increased scatter by comparing the distribution of HRs $\left(\Delta \mu=\mu_{\text {obs }}-\mu_{\text {WMAP }}\right)$ in the "spec Ia" subsample to our full photometric sample, assuming the latest $W M A P+\mathrm{BAO}+H_{0}$ best-fit cosmological model (Jarosik et al. 2011). We show these residuals in three redshift bins of width $\Delta z=0.1$ over the redshift range $0.1<z<0.4$, which corresponds to the range of redshifts where these two sets of SNe Ia significantly overlap. The blue histograms show the "spec Ia" subsample (208), while the 


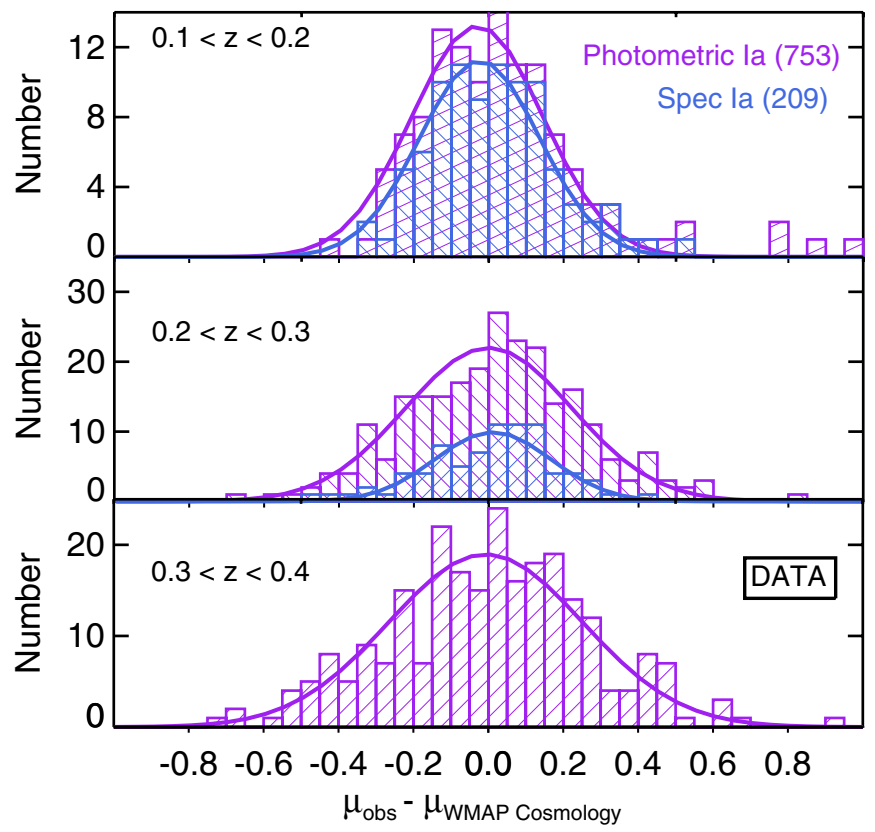

Figure 22. Distribution of Hubble residuals as a function of redshift for the "spec Ia" subsample (blue) and full photometric sample of $752 \mathrm{SNe}$ Ia (purple). (A color version of this figure is available in the online journal.)

Table 3

Parameters of the Best-fit Gaussian Distributions to the Data Shown in Figure 22

\begin{tabular}{lcccr}
\hline \hline \multirow{2}{*}{$\begin{array}{l}\text { Redshift } \\
\text { Bin }\end{array}$} & Sample & \multicolumn{2}{c}{ Gaussian Fit } & \multirow{2}{*}{ Number } \\
\cline { 3 - 4 } & Type & FWHM & Centroid & \\
\hline $0.1<z<0.2$ & spec Ia & $0.377 \pm 0.006$ & $-0.008 \pm 0.008$ & 91 \\
& photo Ia & $0.413 \pm 0.008$ & $-0.012 \pm 0.013$ & 124 \\
\hline $0.2<z<0.3$ & spec Ia & $0.366 \pm 0.010$ & $0.010 \pm 0.012$ & 80 \\
& photo Ia & $0.524 \pm 0.010$ & $0.000 \pm 0.012$ & 249 \\
\hline $0.3<z<0.4$ & photo Ia & $0.610 \pm 0.016$ & $-0.005 \pm 0.016$ & 251 \\
\hline
\end{tabular}

purple histograms include the full 752 photometrically classified $\mathrm{SNe}$ Ia, i.e., blue plus black points from Figure 21.

To quantify the trends we see in Figure 21 (increased dispersion of the photometric SNe Ia with redshift), we fit the HR distributions with Gaussians, and report their FWHM and centroids in Table 3. We fit Gaussians to avoid our analysis being adversely affected by the small but noticeable tails in these distributions, which are likely non-Ia SN contaminants and are clearly offset from the Hubble diagram (Figure 21) at $z \simeq 0.15$.

In Table 3, we see that the centroids of the best-fit Gaussians to both samples of SNe Ia are consistent with zero, showing no bias in their HR distributions as a function of redshift. However, the FWHM of the best-fit Gaussian does increase with redshift for the full photometric sample, and is additionally larger than the FWHM of the "spec Ia" subsample. The quoted errors in Table 3 are given by GAUSSFIT in IDL, and have been confirmed through bootstrap resampling of the distributions (with replacement). We do not report the FWHM for the high-redshift bin of the "spec Ia" subsample as it is unreliable due to small number statistics.

We investigate why this trend appears in the full photometric sample, but not in the "spec Ia" subsample. We plot in Figure 23 the maximum $r$-band $\mathrm{S} / \mathrm{N}$ (at any epoch) for each SN Ia in our observed (left panel) and simulated (right panel) photometrically classified samples. It is clear from the left panel of Figure 23

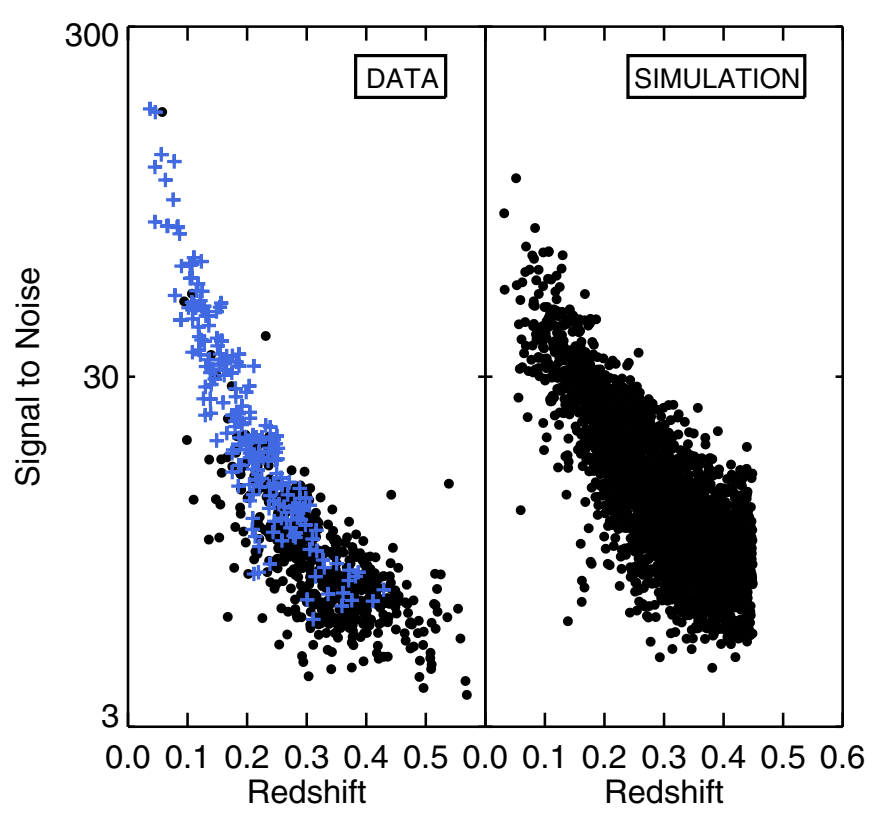

Figure 23. Maximum observed $r$-band $\mathrm{S} / \mathrm{N}$ (at any epoch) from both the observed (left-hand panel) and simulated (right-hand panel) light curves, as a function of redshift. The data sample is divided into SNe Ia with (blue) and without (black) spectroscopic confirmation.

(A color version of this figure is available in the online journal.)

that $\mathrm{SNe}$ in the "spec Ia" (blue) subsample, at a given redshift, possess systematically higher $\mathrm{S} / \mathrm{N}$ light curves than photometrically classified SNe Ia that were not spectroscopically observed (black). The average $\mathrm{S} / \mathrm{N}$ for the "spec Ia" subsample is 27.4, whereas the SNe Ia with only photometric classification have an average $\mathrm{S} / \mathrm{N}$ of 9.6. This is of course expected, as the SDSS-II $\mathrm{SN}$ spectroscopic follow-up observations preferentially selected SN candidates that were easier to observe, naturally leading to a bias in $\mathrm{S} / \mathrm{N}$ for the spectroscopic sample. Thus the "spec Ia" subsample has a smaller scatter because it contains the brightest $\mathrm{SNe}$ Ia from the whole population, which are then easier to fit and thus produce tighter distance modulus constraints. At $z>0.3$, we see in Figure 23 the emergence of an apparent detection limit at $\mathrm{S} / \mathrm{N} \simeq 4-5$. We have determined that this limit is due to the $X_{1}$-color cut (Section 3.1.5), as the SALT2 parameters are not well determined for such noisy light curves, frequently returning unphysical derived parameters which we then exclude.

\section{COSMOLOGY ANALYSIS}

\subsection{Fitting Issues}

To allow consistent comparisons with SNLS, we use the same two methods to perform cosmological fits to our data as used in Guy et al. (2010) and Sullivan et al. (2011). First, we use a grid-based search technique when fitting simple cosmological models to just our photometrically classified SN Ia data. Specifically, we use the simple_cosfitter (Conley et al. 2011) software package, which computes the $\chi^{2}$ at every point in a regular grid (101 by 101) and converts those measurements to a probability via $P \approx \exp \left(-\chi^{2} / 2\right)$, where the proportionality is set by normalizing over the grid. As in the SNLS analysis of Guy et al. (2010) and Sullivan et al. (2011), we then marginalize over the SALT2 SN parameters $(\alpha, \beta, M)$ to generate confidence contours for the cosmological parameters of interest.

We also use the CosmoMC (Lewis \& Bridle 2002) software package when fitting more complex cosmological models to our 
Table 4

Priors Imposed on the Fitted Cosmological Parameters in Four Different Combinations (Sets)

\begin{tabular}{lccccc}
\hline \hline \multirow{2}{*}{ Parameter } & \multicolumn{2}{c}{ Simple_cosfitter } & & \multicolumn{2}{c}{ CosmoMC } \\
\cline { 2 - 3 } \cline { 5 - 6 } & Set I & Set II & & Set III & Set IV \\
\hline$w$ & -1 & -1 & & $-3,3$ & $-3,3$ \\
$\Omega_{\mathrm{m}}$ & $0.0,1.5$ & $0.0,1.5$ & & - & - \\
$\Omega_{\Lambda}$ & - & $-0.5,2.5$ & & - & - \\
$\Omega_{\mathrm{k}}$ & 0 & - & & 0 & $-1.5,1.5$ \\
$\Omega_{\mathrm{dm}}$ & - & - & & $0.0,1.2$ & $0.0,1.2$ \\
$\Omega_{\mathrm{b}}$ & - & - & & 0.0458 & $0.015,0.200$ \\
$H_{0}$ & - & - & & 50,100 & 50,100 \\
\hline
\end{tabular}

Notes. The dashed symbol in the table represents where we do not need to set priors for parameters, as they are constrained by a combination of other priors (e.g., $\Omega_{\mathrm{m}}$ is restricted by the priors on $\Omega_{\mathrm{dm}}$ and $\Omega_{\mathrm{b}}$ ).

data (and simulations; see Section 3.1.1) in combination with other cosmological information. This package uses the Markov Chain Monte Carlo (MCMC) technique to efficiently probe multi-dimensional parameter space, allowing one to quickly investigate a large number of different regions in the parameter space. We have slightly modified CosmoMC to allow for the simultaneous fitting of both the cosmological parameters and the SALT2 SN parameters $\alpha$ and $\beta$, which define the standardization of SNe Ia. We include in the distance modulus calculation the redshift-dependent Malmquist bias correction (Equation (4)) and the full SALT2 light-curve parameter covariance matrix. Finally, at each point in the MCMC chain, we determine $M$ (absolute magnitude at peak for $\mathrm{SNe}$ Ia) for the value of $H_{0}$ at that step in the chain. This approach is the same as analytically marginalizing over $M$, as outlined in Bridle et al. (2002), and is a similar methodology as used by SNLS (Sullivan et al. 2011). This method is used in all our cosmological fits.

We execute our modified CosmoMC code using six chains in parallel to facilitate quick coverage of the large, multidimensional parameter space. Each chain is started at a random location within our defined parameter space and typically converges after 50,000 to 100,000 steps. We assume that the MCMC has converged when $R-1<0.1$, where $R$ is the Gelman and Rubin statistic, i.e., when the variance within the chains is equal to the variance between chains (Brooks \& Gelman 1998).

We provide in Table 4 a summary of the different combinations of priors we assume during our different cosmological fits discussed below. Throughout all our analyses we assume flat distributions for the priors on our $\mathrm{SN}$ parameters, i.e., $\alpha=(0.01,0.5)$ and $\beta=(1.0,5.0)$. We also assume flat distributions for the priors on $\Omega_{\mathrm{dm}}$ (density of dark matter) and $\Omega_{b}$ (density of baryonic matter) when these cosmological parameters are allowed to vary.

Finally, we set the value of the intrinsic dispersion of SNe Ia $\left(\sigma_{\text {int }}\right)$ to $\sigma_{\text {int }}=0.12 \mathrm{mag}$, which is then added in quadrature to all our SN errors. Although this results in a reduced $\chi^{2}$ close to unity for all our cosmological fits, the best-fit value (i.e., delivers the reduced $\chi^{2}$ closest to one) for all our data is 0.16 mag. However, our simulations show that this larger value of $\sigma_{\text {int }}$ is consistent with our small level of non-Ia SN contamination, ${ }^{36}$ i.e., we input $\sigma_{\text {int }}=0.12$ mag in our simulation, but measure

\footnotetext{
36 We find that the intrinsic dispersion of our full sample drops to $\sigma_{\text {int }}=0.1$, and our cosmological results remain consistent, if we simply remove the 25 $\mathrm{SNe}$ Ia that are located $>3 \sigma$ away from the best-fit cosmological model. We do not recommend such a "sigma-clipping" technique when using $\mathrm{SNe}$ Ia to test cosmological models, but this test does illustrate the sensitivity of $\sigma_{\text {int }}$ to such outliers.
}

Table 5

Summary of the Cosmological Fits Presented in Section 6

\begin{tabular}{|c|c|c|c|c|c|c|c|c|}
\hline \multicolumn{5}{|c|}{ Data } & \multicolumn{4}{|c|}{ Results } \\
\hline $\mathrm{SNe}$ & $H_{0}$ & CMB & LRGs & Priors & $\Omega_{\mathrm{m}}$ & $\Omega_{\Lambda}$ & $w$ & Figure \\
\hline$X$ & & & & Set I & $0.24_{-0.05}^{+0.07}$ & $\cdots$ & $\cdots$ & $\cdots$ \\
\hline $\mathrm{X}$ & $X$ & & & Set III & $0.27_{-0.16}^{+0.15}$ & $0.73_{-0.15}^{+0.16}$ & $-0.95_{-0.32}^{+0.31}$ & 25 \\
\hline $\mathrm{X}$ & $\mathrm{X}$ & $X$ & $X$ & Set IV & $0.29_{-0.02}^{+0.02}$ & $0.71_{-0.02}^{+0.02}$ & $-0.96_{-0.10}^{+0.10}$ & 26 \\
\hline
\end{tabular}

$\sigma_{\text {int }}=0.16$ mag for a photometrically classified sample like ours. As $\sigma_{\text {int }}$ is used to explain unknown residual scatter in the $\mathrm{SNe}$ Ia population, we feel it is appropriate to remove any extra scatter caused by non-Ia SN contamination from our "measured" value of $\sigma_{\text {int }}$. We stress that this statement is not in contradiction with Section 5.1, where we state that the larger scatter we observe in our sample could be caused by lower $\mathrm{S} / \mathrm{N}$ light curves, as such lower-quality data would increase both the scatter in the population and the observed errors bars on our SN distance moduli. However, contamination would increase the overall scatter in the SN HRs without necessarily increasing the distance uncertainties. It is worth noting that we also ran our cosmology fits with the $\sigma_{\text {int }}=0.16$ mag and found no significant difference to our cosmological results.

We note that our assumed value of $\sigma_{\text {int }}=0.12 \mathrm{mag}$ is still higher than that found in Lampeitl et al. (2010a, $\sigma_{\text {int }}=$ $0.088 \mathrm{mag}$ ) and in Kessler et al. (2009a, $\sigma_{\text {int }}=0.08 \mathrm{mag}$, SALT2 and SDSS-II SNe). These values were computed for the first-year SDSS SN Survey spectroscopic sample; limiting ourselves to the spectroscopically confirmed subsample of our full photometric sample (Section 5), we still find a larger intrinsic scatter, $\sigma_{\text {int }}=0.11 \mathrm{mag}$. However, Conley et al. (2011) found $\sigma_{\text {int }}=0.10 \mathrm{mag}$ for the SDSS spectroscopically confirmed data, in agreement with our value. The apparent discrepancy may be caused by Kessler et al. (2009a) and Lampeitl et al. (2010a) using an older version of SALT2 (Guy et al. 2007), as Conley et al. (2011) also use the same newer version of SALT2 (Guy et al. 2010) used here. As our simulations have shown that our selection criteria do not result in a bias between the input and best-fit value of $\sigma_{\text {int }}$ for a pure (i.e., spectroscopic) sample of SNe Ia, we have confidence in and continue to use throughout our value of $\sigma_{\text {int }}=0.12 \mathrm{mag}$. We stress that the assumed value of $\sigma_{\text {int }}$, within the range discussed above, has little effect on the cosmological fits presented in this paper as the fits also have the freedom to adjust the values of other SN parameters, such as $\alpha$ and $\beta$.

\subsection{Our Constraints on $\Lambda C D M$}

We begin by studying the cosmological constraints obtained using only our SN Ia sample, before combining with other data. For completeness, we provide a summary of all our cosmological fits, including the different combinations of data sets and priors, in Table 5.

We begin by fitting the $\Lambda$ CDM cosmological model $(w=-1)$ to our photometrically classified SNe Ia using simple_cosfitter and prior Set I in Table 4. Under the assumption of flatness we obtain a best-fit value of $\Omega_{\mathrm{m}}=0.24_{-0.05}^{+0.07}$ (statistical errors only). When we relax the prior on flatness (prior Set II), we obtain the (gray) confidence contours for $\Omega_{\mathrm{m}}$ and $\Omega_{\Lambda}$ in Figure 24. For comparison, we show similar constraints on these cosmological parameters using the three-year SNLS data (SNLS3) from Guy et al. (2010), which only includes 242 spectroscopically classified SNe Ia from SNLS. 


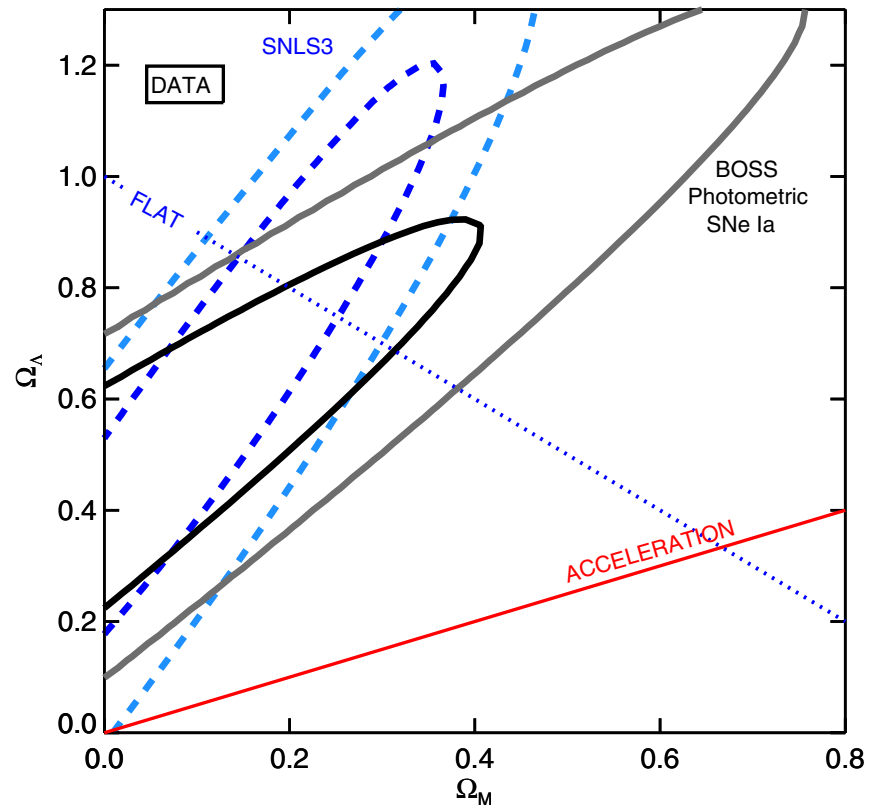

Figure 24. $68 \%$ and $95 \%$ confidence contours of $\Omega_{\mathrm{m}}$ vs. $\Omega_{\Lambda}$ for a $\Lambda$ CDM model using only our photometrically classified SNe, with prior Set II (Table 5), and allowing curvature to vary. Only statistical errors in the contours are shown. The blue dashed contours show the comparable SNLS3 constraints taken from Guy et al. (2010).

(A color version of this figure is available in the online journal.)

Figure 24 also demonstrates that our photometrically classified SN Ia sample alone is able to detect an accelerating universe (i.e., $\Omega_{\Lambda}>\Omega_{\mathrm{m}} / 2$ ). Integrating over the whole parameter space in Figure 24 (see Table 4 for parameter ranges), we compute the probability of an accelerating universe given our data to be $99.96 \%$ (statistical uncertainties only).

\subsection{Our Constraints on $w C D M$}

We next fit for a flat, $w$ CDM cosmological model using CosmoMC and the prior Set III in Table $4 .{ }^{37}$ We fit this model to our sample of photometrically classified SNe Ia and the $H_{0}$ measurement of $73.8 \pm 2.4 \mathrm{~km} \mathrm{~s}^{-1} \mathrm{Mpc}^{-1}$ from the recent "Supernovae and $H_{0}$ for the Equation of State" sample (SH0ES; Riess et al. 2009, 2011). This Gaussian prior on $H_{0}$ does not impact the cosmological constraints due to our marginalization over the absolute magnitude of $\mathrm{SNe}$ Ia, but rather ensures that cosmoMC, which assumes a minimum age to the universe, performs in a well-behaved manner. We note that within cosmoMC, $\Omega_{\mathrm{m}}$ is a parameter comprised of two components: $\Omega_{\mathrm{b}}$ and $\Omega_{\mathrm{dm}}$. Since the ratio of these two components is not constrained by SNe Ia alone, we fix $\Omega_{\mathrm{b}}$ to the WMAP7 value of 0.0458 (Jarosik et al. 2011), reducing the number of parameters to be constrained by the SNe data to two: $\Omega_{\mathrm{dm}}$ and $\mathrm{w}$.

We find the best-fit values for this model to be $w=$ $-0.95_{-0.31}^{+0.32}$ and $\Omega_{\mathrm{m}}=0.27_{-0.16}^{+0.15}$ (statistical errors only), and show in Figure 25 the joint $68 \%$ and $95 \%$ confidence intervals for these data. We also show the equivalent contours for the three-year sample of spectroscopically confirmed $\mathrm{SNe}$ Ia from the SDSS-II SN Survey. For this comparison, we include all 306 confirmed SNe Ia (regardless of whether they are part of our photometric sample or not) at a redshift of

\footnotetext{
37 All other cosmological parameters are left at their default values (i.e., re-ionization optical depth, the primordial super-horizon power in the curvature perturbation on $0.05 \mathrm{Mpc}^{-1}$ scales, and the scalar spectral index).
}

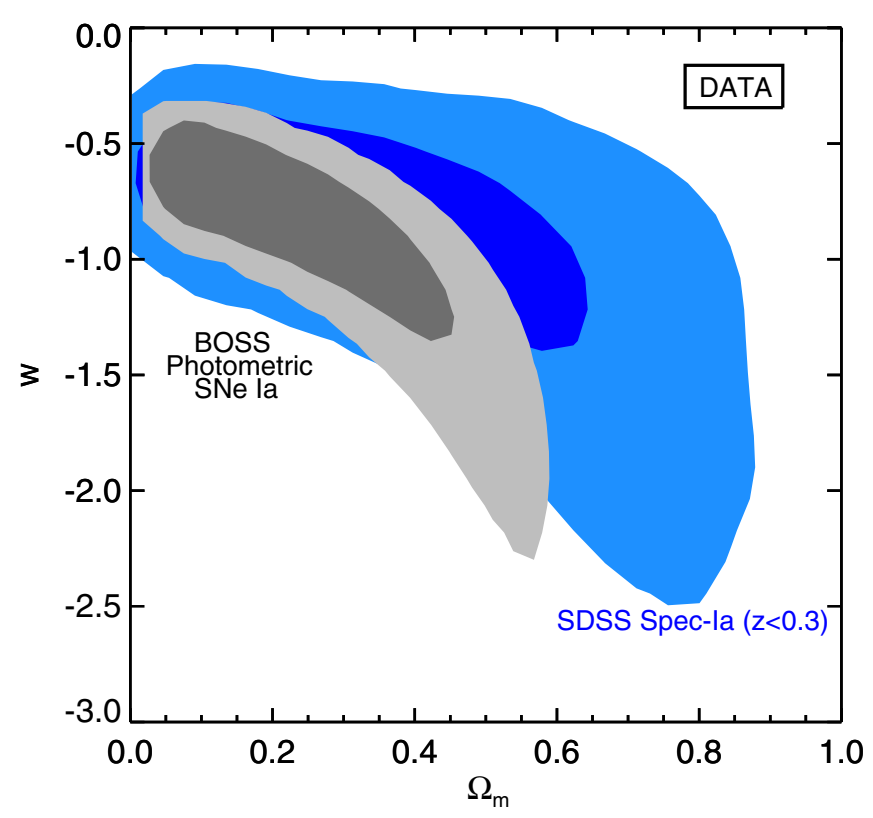

Figure 25. $68 \%$ and $95 \%$ confidence contours of $w$ and $\Omega_{\mathrm{m}}$ in a flat $w$ CDM model (assuming prior Set III in Table 5) for a combination of our photometrically classified sample of $752 \mathrm{SNe}$ Ia and the SHOES measurement of $H_{0}$ (only the statistical errors shown, gray). The blue contours show the equivalent constraints but using the three-year sample of spectroscopically confirmed SNe Ia from the SDSS-II SN Survey (confined to $z<0.3$ ).

(A color version of this figure is available in the online journal.)

$z<0.3$, below which any selection bias in the spectroscopic sample should be minimized. This comparison demonstrates that using photometric instead of spectroscopic classification, which increases the size of the sample by a factor of 2.5 and extends the redshift range, results in a reduction of the area of the confidence contours by a factor of 1.6. We stress that this simplistic comparison does not constitute a detailed analysis of the full three-year spectroscopic sample of the SDSS-II SN Survey, which will be presented elsewhere. We also find that limiting our photometric SNe Ia sample to $z<0.3$ gives consistent cosmological constraints with the samples plotted in Figure 25, but with slightly larger uncertainties.

During this analysis, we simultaneously solve for the best-fit values of the SALT2 SN parameters. In our fit of the $w$ CDM model, we find $\alpha=0.22_{-0.02}^{+0.02}$ and $\beta=3.12_{-0.12}^{+0.12}$. Our fitted value for $\beta$ is in agreement with previous analyses of the SDSS data (Lampeitl et al. 2010a; Marriner et al. 2011; Conley et al. 2011). However, our value of $\alpha$ appears higher than previous analyses; Lampeitl et al. (2010a) found $\alpha=0.16 \pm$ 0.03 , and Marriner et al. (2011) found $\alpha=0.131_{-0.04}^{+0.05}$. One potential explanation of this difference could be the non-Ia SNe contamination in our photometric sample, but this has been tested in our simulations: we recover the input $\alpha$ and $\beta$ both with and without the expected level of contamination detailed in Section 3. A more plausible explanation appears to be the higher average $\mathrm{S} / \mathrm{N}$ of the spectroscopically confirmed $\mathrm{SNe} I \mathrm{I}$; using only the subset of spectroscopically confirmed SNe Ia in our photometric sample, we find $\alpha=0.16 \pm 0.02$, in agreement with previous results.

We explored whether HR outliers (which we do not automatically clip) might be affecting the derived value of $\alpha$. We subtracted $\alpha * X_{1}$ from the HR of each SN in our sample and plotted these as a function of $X_{1}$, finding four clear outliers. Removing these objects from our sample and refitting the cosmology, we found the resulting cosmological contours to be 


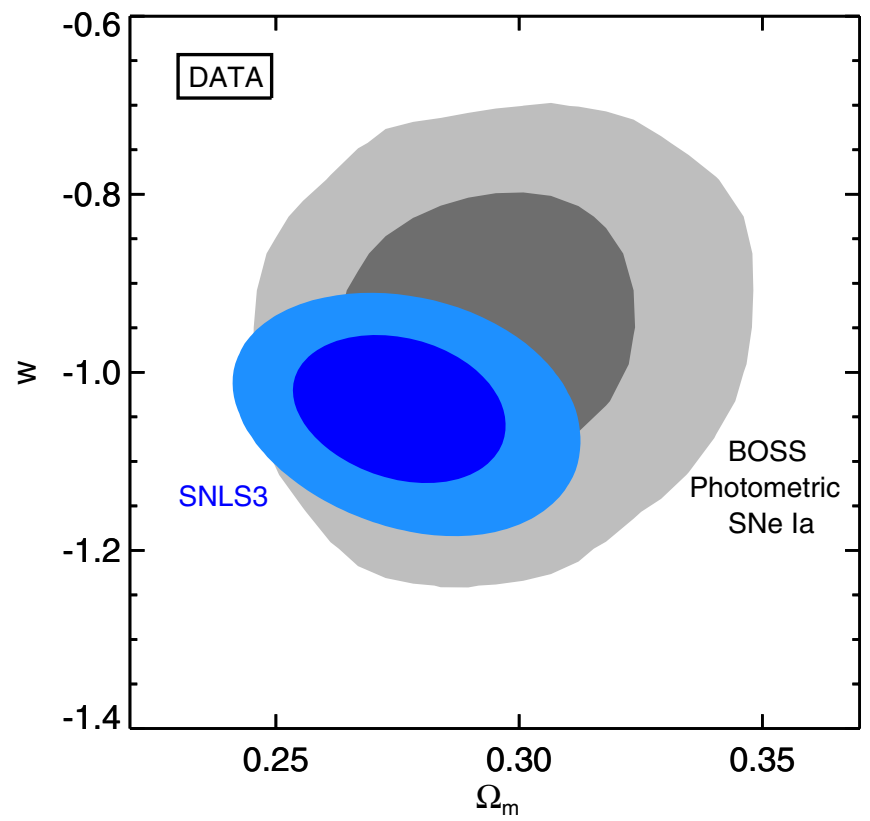

Figure 26. $68 \%$ and $95 \%$ confidence contours of $w$ and $\Omega_{\mathrm{m}}$ in a $w \mathrm{CDM}$ model from the spectroscopic SNLS3 sample (Sullivan et al. 2011, blue) and our photometrically classified SDSS-II SN Ia sample (gray) using prior set IV in Table 5. Both sets of contours include external data from the CMB, LRGs, and the SHOES $H_{0}$ measurement. Contours represent statistical uncertainties only.

(A color version of this figure is available in the online journal.)

unchanged but the derived SALT2 parameters to both be lower $(\alpha=0.18, \beta=2.79)$. As this $\alpha$ is still larger than what is found in previous studies, the outliers cannot be solely responsible for this discrepancy, and regardless does not result in biased cosmological constraints.

\subsection{Constraints from Combining Data Sets and Comparison with SNLS}

Finally, we determine cosmology constraints with our photometrically classified sample of 752 SNe Ia combined with cosmological information from the power spectrum of luminous red galaxies (LRGs) in the SDSS DR7 (Reid et al. 2010), the full WMAP7 CMB power spectrum (Larson et al. 2011), and the SHOES $H_{0}$ measurement. The SHOES $H_{0}$ measurement is partially determined using nearby SNe Ia measurements, and thus to be fully consistent we would have to consider the covariance between this value of $H_{0}$ and our SNe Ia measurements. However, as we are assuming no prior information on $M$ in our treatment of intrinsic SN parameters, these measurements can be considered independent. Furthermore, the uncertainty in $M$ is a subdominant systematic uncertainty to the derived value of $H_{0}$ (Riess et al. 2011).

We fit this combination of data using CosmoMC for a non-flat $w$ CDM cosmology, using the priors listed as Set IV in Table 4. With the addition of these external data sets, we can now relax our priors on the re-ionization optical depth $(\tau=[0.00,0.50])$, the primordial super-horizon power in the curvature perturbation on $0.05 \mathrm{Mpc}^{-1}$ scales $(\log A=[0,30])$, and the scalar spectral index $\left(n_{\mathrm{S}}=[0,1.5]\right)$.

We find the best-fit value for the equation of state of dark energy using these data is $w=-0.96_{-0.10}^{+0.10}$, with $\Omega_{\mathrm{m}}=0.29_{-0.02}^{+0.02}$ and $\Omega_{\mathrm{k}}=0.00_{-0.01}^{+0.01}$ (statistical errors only). We also find a best-fit value of $H_{0}=67.97_{-2.25}^{+2.28}$ (stat) $\mathrm{km} \mathrm{s}^{-1} \mathrm{Mpc}^{-1}$. These cosmological constraints are summarized in Table 5.
Figure 26 shows our joint confidence contours for $w$ and $\Omega_{\mathrm{m}}$ in comparison with similar SNLS3 constraints from Sullivan et al. (2011) using the same combination of external data-sets (CMB, LRGs, and the $H_{0}$ SHOES). SNLS3 only uses spectroscopically classified SNe Ia, collected from the following SN data sets: 242 SNe Ia from SNLS; 123 low-redshift SNe Ia from the literature (primarily Hamuy et al. 1996a; Riess et al. 1999; Jha et al. 2006; Hicken et al. 2009a; Contreras et al. 2009); 14 high-redshift SNe Ia from Hubble Space Telescope (HST; Riess et al. 2007); and 93 SNe Ia over the redshift range $0.06 \leqslant z \leqslant 0.4$ from the first-year SDSS SN Survey (Kessler et al. 2009a). The inclusion of the SDSS SN data means there are some SNe Ia in common between these analyses. There appears to be good agreement in these two sets of constraints, although there is a small offset in the bestfit values for $\Omega_{m}$ between the two analyses: $\Delta \Omega_{m}=0.018$, significant at less than $1 \sigma$. The best-fit values of $w$ differ by $\Delta w=0.080$ between the two analyses, which is again consistent within the quoted uncertainties. One possible explanation for these small differences is the fact that we have not corrected for the correlation with host-galaxy stellar mass, as discussed in Section 7. Overall this comparison is reassuring, considering the lower redshift leverage of the SDSS-II SN sample $(z<0.55)$ and the lack of spectroscopic confirmation used herein. These results demonstrate the potential for photometrically classified SN Ia samples to be used to improve cosmological constraints in the future.

\section{DISCUSSION}

\subsection{Systematic Uncertainties in Photometric Classification}

The goal of this paper has been to illustrate the power of photometrically classified $\mathrm{SNe}$ Ia in delivering competitive cosmological results when compared to spectroscopically confirmed samples. As such, we have paid particular attention to the sources of uncertainty unique to our methodology of SN classification, and to the SDSS-II SN Survey in general, i.e., contamination from non-Ia SNe and Malmquist bias. We have not undertaken an exhaustive study of systematic uncertainties as exemplified by Kessler et al. (2009a) and Conley et al. (2011).

In Section 4, we studied the effect of non-Ia SN contamination of photometric samples on cosmological constraints. The low predicted contamination rate $(3.9 \%)$ in our sample has an insignificant effect on the best-fit cosmological parameters in simulations; compared to a completely pure sample of $\mathrm{SNe}$ Ia we find a bias in the equation of state of only $w \simeq 0.007$ (using prior Set III in Table 4).

We have also studied the systematic offset associated with the Malmquist bias effect (Section 4.1). This correction, as a function of redshift, is included in all our cosmological fits. We also investigated allowing the three parameters in the Malmquist bias exponential parameterization (Equation (4)) to vary in the cosmological fit within the error bars; the cosmological constraints remained identical. Correlations between the three parameters in the Malmquist bias fit have not been investigated in this paper.

We do not attempt to correct for the known correlation between the SN Ia HRs and the properties of their host galaxies; SNe Ia in massive galaxies are overluminous even after light-curve corrections. Our sample of host galaxies spans a range in absolute magnitude of approximately $-24<$ $M_{r}^{0.1}<-18$ (Figure 20), which corresponds to a stellar mass range of $\simeq 10^{9}-10^{11} M_{\odot}$, or a predicted difference of $\Delta \mu \simeq 0.1$ (Lampeitl et al. 2010b). We additionally expect that 
the magnitude limit imposed on our host-galaxy selection could cause a bias by preferentially selecting more massive (more luminous) host galaxies at higher redshift, thus preferentially selecting overluminous SNe Ia. Unfortunately, the underlying physical mechanism that drives this correlation remains unclear (e.g., D'Andrea et al. 2011; Gupta et al. 2011), and as such the mass-HR relationship could also be subject to a redshift dependence. Therefore, we do not correct for this effect here, but note that it will be essential for future studies. We note that introducing a correction term for host-galaxy properties is no more difficult in photometrically classified SN Ia samples than in those obtained from spectroscopic follow-up, so it is a common problem for all future SN surveys.

A systematic uncertainty that is unique to photometrically classified SN Ia samples like ours, based on host-galaxy spectroscopy, are errors in associating the $\mathrm{SNe}$ with the correct host galaxy. As described in Appendix C, we found only one mismatch between the SDSS-II SN spectroscopic subsample and our photometrically classified SNe Ia, one mismatch between the SDSS-II host-galaxy spectroscopy and BOSS host-galaxy spectroscopy, and giving an error rate of only $0.6 \%$. We have removed this object, as well as another likely matching error discussed in Section 3.2, from our photometric sample. We cannot rule out the possibility that other SN-galaxy pairs have been incorrectly matched, although based on the rate found in the spectroscopic subsample we expect the number to be low and thus have a negligible effect on our cosmological fits.

Finally, our estimation of systematic effects due to Malmquist bias and contamination relies on the assumption that our simulations accurately represent the final sample after selection cuts. However, some implicit assumptions in the simulation may not be valid. For example, we assume that all candidates are SN Ia or non-Ia SNe, even though some photometrically classified candidates may in fact be another type of transient (e.g., AGN). We also assume that the 41 non-Ia templates reflect a complete sample of non-Ia SNe, and that the non-Ia properties are redshift independent. These assumptions may be inadequate, as discussed further in Section 7.2 and Kessler et al. (2010b).

The magnitude of these systematic biases on our results is either small compared to our statistical uncertainties or unknown, and as such we do not include them in the error budget of our derived cosmological parameters in Table 5.

\subsection{Future Improvements to Photometric Classification}

We have provided in this paper a procedure for photometrically classifying $\mathrm{SNe}$. Of course, other methods also exist, with their own relative advantages and disadvantages. For example, rather than applying limits on light-curve properties in two-dimensional parameter space (e.g., Figure 6), one could apply a nearest-neighbor algorithm to look for clustering in higher dimensional parameter space. Alternatively, we note that the analysis presented here excludes from our sample all transients that have $P_{\text {Ia }}$ computed to be below a hard threshold (see Section 3.1.4). One could choose instead to retain all SN candidates in their cosmology analysis, weighting each candidate by its Bayesian probability $\left(P_{\mathrm{Ia}}\right)$ of being an SN Ia. This approach avoids the uncertainty of choosing the optimal $P_{\text {Ia }}$ threshold to obtain a "clean" sample of SNe Ia, and prevents the removal of actual SNe Ia and the information that they provide. These methods - the nearest-neighbor algorithm and the fully Bayesian method using the BEAMS algorithm (Hlozek et al. 2012) - are currently being investigated using the SDSS-II SN candidates (and their BOSS host-galaxy redshifts).
Although we have optimized the selection cuts implemented in this paper, utilizing higher-order criteria could further improve the accuracy and efficiency of photometric classification. For example, the ellipsoidal $X_{1}$-color cut could be derived with an additional rotation parameter that accounts for the correlations between the two SALT2 light-curve parameters.

There is also room for improvement in the simulations we use to describe our observations and measure the efficacy of our selection criteria. The SN Ia templates included in the simulations are missing a number of subclasses, such as 02cx-like SNe, which have similar light curves to but are fainter than normal SNe Ia (Li et al. 2003; Phillips et al. 2007; McClelland et al. 2010); 06bt-like SNe, which are particularly problematic (Foley et al. 2010b; Stritzinger et al. 2011); and super-Chandrasekhar mass SNe Ia (Howell et al. 2006; Scalzo et al. 2010). The simulations also include only a limited number of non-Ia SN templates, a deficiency which is sure to be improved in the future as the quantity of observations of these objects begins to reflect their diversity.

At present, the only transient objects included in these simulations are $\mathrm{SNe}$ (Ia, II, and Ibc). In the future we would like to include other transient objects that are known contaminants in SN surveys, such as AGNs. Therefore, when we quote an estimated contamination of $3.9 \%$, this could be underestimated as we have only included contamination from non-Ia SNe. Furthermore, errors on assigning the correct $\mathrm{SN}$ host redshift were not modeled in our simulations. This is a rather complicated effect, as it depends on both the distribution of $\mathrm{SNe}$ within galaxies and the luminosity function of galaxies (those with and without $\mathrm{SNe}$ ) as a function of redshift. While we believe we have removed possible misidentified host galaxies from our data (Appendix C), it would be interesting to model whether the expected number of such misidentifications is consistent with our findings.

In this work we have assumed a constant value for the intrinsic dispersion of our sample. However, Kessler et al. (2012) have recently shown that the intrinsic dispersion of SDSS and SNLS SN data may be better described as a wavelength-dependent function. Therefore, future analyses will want to include a more complex model than is currently standard for the intrinsic dispersion to improve their cosmological constraints.

We note that the relative rates as a function of redshift of SNe Ia (based on Dilday et al. 2008) and non-Ia SNe (Bazin et al. 2009) assumed in the simulations still have associated uncertainties. It is certain that these constraints will be improved in the near future by the next generation of large, deep SN surveys.

Finally, in this paper we have used a simple redshiftdependent correction for the Malmquist bias. In the future, higher-order Malmquist bias corrections could be investigated, such as stretch-dependent or color-dependent corrections. The latter of these may be important, as Figure 15 shows there is a clear bias in the recovered color distribution with redshift. The ESSENCE survey used a color-dependent Malmquist bias correction, adjusting the prior on the host-galaxy extinction $\left(A_{v}\right)$ as a function of redshift (Wood-Vasey et al. 2007). This method would be interesting to explore with our photometrically classified SNe Ia.

\subsection{Prospects for Future SN Surveys}

The DES Supernova Survey (Bernstein et al. 2012) should start by the end of 2012 and run for at least five seasons. It is expected to measure high-quality light curves for $\sim 4000 \mathrm{SNe}$ Ia 
out to a redshift of $z \approx 1.2$, for which real-time spectroscopy of every SN Ia candidate, as done previously, will be impractical. The SNLS, SDSS, and ESSENCE surveys combined used over a year of telescope time (4 and $8 \mathrm{~m}$ class) to spectroscopically confirm fewer than 1000 SNe Ia (Foley et al. 2009; Howell \& Legacy Survey 2009).

Therefore, DES will need to use photometric classifications to reduce the burden of real-time spectroscopic confirmation and use allocated spectroscopic resources wisely, e.g., targeting hostless $\mathrm{SNe}$ for spectroscopic confirmation or building up training samples of SNe for photometric classifiers. Our work suggests the need for obtaining spectroscopy of SN host galaxies which, as was the case of SDSS-II, can be done over a longer period of time and can be coordinated with other science goals (Lidman et al. 2012).

Photometric classification is well suited for obtaining large, uniformly selected samples of SNe Ia. In light of the systematics discussed in Section 7.1 and Appendix D, there is a great scientific benefit in having such samples that can be subdivided and analyzed a number of different ways to determine the magnitude of such systematic effects. We describe below two examples of how these samples will be useful.

SN lensing is the increase in observed flux from an SN due to lensing by the structure the light passes through on its journey through the universe. Clarkson et al. (2012) have discussed how the number of galaxies we observe along the line of sight to an SN should be correlated with the overluminosity of the SN (voids play a role in this determination as well). Thus grouping large numbers of SNe Ia by the amount of foreground structure is vital for measuring this effect; such a program is currently being investigated with this sample (M. Smith et al., in preparation).

The correlation between SN Ia host-galaxy properties (mass, star formation rate, metallicity) and SN Ia HRs after light curve and color corrections are made is an important discovery for SN Ia cosmology (Gallagher et al. 2008; Hicken et al. 2009b; Kelly et al. 2010; Sullivan et al. 2010; Lampeitl et al. 2010b; Gupta et al. 2011; D'Andrea et al. 2011). Using spectroscopically confirmed SDSS-II SNe, Lampeitl et al. (2010b) showed that SNe Ia are $\sim 0.10$ mag intrinsically overluminous in passive hosts, and also found that parameterization using stellar mass gives an improvement of $\sim 4 \sigma$ on the cosmological constraints. This effect, however, is most likely not driven by the host mass; using low- $z$. SDSS-II SNe Ia, D'Andrea et al. (2011) found intrinsically overluminous $\mathrm{SNe}$ occur in high-metallicity galaxies at $>3 \sigma$. The recent SNLS3 cosmology analysis (Sullivan et al. 2011) is the first major SN study to include host-galaxy corrections in a full cosmology analysis.

Our SDSS-II photometric SN Ia sample is much larger than any previously analyzed sample, and the improved statistics and reduced bias may lead to an improved understanding of this effect. Correlations with photometrically derived stellar mass and spectroscopically derived metallicities and star formation rates are typically degenerate, but a large sample will allow one to hold these parameters constant while allowing only one to vary.

\section{CONCLUSIONS}

In this paper we use the full three-year photometry from the SDSS-II SN Survey, together with BOSS spectroscopy of the host galaxies of transients, to create a photometrically classified sample of SNe Ia to be used for cosmology. Our main results are as follows.
1. We have created a homogeneous sample of 752 photometrically classified $\mathrm{SNe}$ Ia; the largest collection of SNe Ia ever selected from a single photometric survey. Our sample spans a redshift range of $0.05<z<0.55$ and contains 544 newly classified SNe Ia; 208 SNe Ia in our sample had been previously spectroscopically confirmed and another 115 had been photometrically classified using SDSS-II hostgalaxy spectra. Based on SNANA simulations, we estimate that this sample is $70.8 \%$ efficient at detecting SNe Ia, with a contamination of only $3.9 \%$ from core-collapse SNe. We demonstrate that this level of contamination is negligible when estimating constraints on cosmological parameters using this sample of SNe Ia.

2. Malmquist bias and SALT2-related effects are the largest systematic selection uncertainties in our photometrically classified SNe Ia sample. We estimate the combined size of these effects using extensive SNANA simulations, which show that they can be $>0.1 \mathrm{mag}$ at the high-redshift limit of our sample ( $z=0.5$; Figure 19). We use our simulations to correct for these biases and show we can then recover the correct cosmological model input into the simulations.

3. We show that the "spec Ia" subsample of $208 \mathrm{SNe}$ Ia is potentially biased, as they possess higher $\mathrm{S} / \mathrm{N}$ light curves than the majority of our photometrically classified SNe Ia (Figure 23). Furthermore, there is evidence in Figures 14 and 15 that the "spec Ia" subsample is biased to brighter (higher stretch) and slightly bluer (lower color values) SNe Ia than the whole population. The weighted means of the SALT2 parameters for the "spec Ia" sample are $X_{1}=0.033 \pm 0.015$ and $c=-0.021 \pm 0.002$, compared to $X_{1}=-0.017 \pm 0.013$ and $c=-0.018 \pm 0.002$ for the sample as a whole. It is additionally clear from Figure 20 that the host galaxies of the "spec Ia" subsample are biased toward fainter (in absolute magnitude) galaxies compared to the whole population of host galaxies. These biases may be related, but we have not investigated this possibly herein.

4. We present the corrected Hubble diagram for our photometrically classified SN Ia sample in Figure 19. The extra scatter seen in this diagram at high redshifts is likely caused by our sample including $\mathrm{SNe}$ Ia with lower $\mathrm{S} / \mathrm{N}$ than are found in the spectroscopically confirmed SNe Ia subsample (based on our simulations). We then fit this Hubble diagram with a straightforward $\Lambda \mathrm{CDM}(w=-1)$ cosmological model to obtain $\Omega_{\mathrm{m}}=0.24_{-0.05}^{+0.06}$ (statistical errors only) for a flat universe. If we relax the constraint on flatness we obtain the constraints on $\Omega_{\mathrm{m}}$ and $\Omega_{\Lambda}$ shown in Figure 24, where we have detected an accelerating universe at the $99.96 \%$ confidence level. This figure also shows that our statistical constraints on these important cosmological parameters are comparable to the recent SNLS three-year constraints published in Guy et al. (2010).

5. In Figures 25 and 26, we show our constraints on the equation of state of dark energy $(w)$ for our photometrically classified sample on its own (with only $H_{0}$ data) and when combined with other cosmological information (CMB, LRGs, $\left.H_{0}\right)$. We find $w=-0.95_{-0.31}^{+0.32}$ and $w=-0.96_{-0.10}^{+0.10}$, respectively (statistical errors only), which are consistent with both the SDSS-II and SNLS three-year spectroscopic samples. These cosmological analyses illustrate that our photometrically classified sample can deliver competitive constraints even though it lacks extensive SN spectroscopic follow-up and probes a smaller range of redshifts (compared to the SNLS3). 
Creating a photometric SN Ia sample is a fundamentally different task from creating a spectroscopic sample. There are various equally valid approaches that can be taken into account in designing an SN Ia photometric-classification algorithm depending on the purpose to be fulfilled, each resulting in a sample of different size and composition. As our focus is on obtaining useful cosmological constraints, this required prioritizing a highly pure sample (few non-Ia SNe included) over a highly efficient one (few SNe Ia excluded). Had our intention been to study, for example, $\mathrm{SN}$ rates, then the selection pressures driving our sample construction would have been different. A focus on cosmology and thus purity will necessitate the sort of strict cuts on photometric properties that we apply.

Our classification is based on the technique of S11, which computes the Bayesian probability of each SN subtype based on the fit of the data to templates and models (Appendix A). However, on its own this method does not perform nearly as efficiently as it does when a prior on the redshift exists for each SN candidate (M. D. Olmstead et al., in preparation). We undertook an ancillary program using BOSS to obtain hostgalaxy redshifts for a large number of SN candidates; this forms a crucial part of our paper. We have argued that obtaining the resources to spectroscopically observe the majority of SNe Ia in future surveys is not feasible. This is because SN spectroscopy is a highly time sensitive (observing windows of a few weeks) and scattered (low density per solid angle per unit time) undertaking. For this reason, it is also subject to significant selection biases. Host-galaxy spectroscopy allows the observer to obtain the redshift of each object much more efficiently: multi-object spectrographs can be used to sample the higher spatial density of targets at scheduled dates long after the SNe have faded away. For this reason we believe host spectroscopy will remain a vital component of SN surveys in the future.

It is not desirable for future surveys to abandon real-time spectroscopy completely; it will remain necessary to identify subtypes of SNe Ia, train classifiers, and study detailed properties. It is even possible for spectroscopic samples with sizes and redshift ranges exceeding that of SNLS and SDSS-II to be created. However, this method could not possibly achieve the volume of $\mathrm{SNe}$ Ia identification possible with photometric classification. And although statistical uncertainties today are quite small, sample size is very important for understanding systematic uncertainties (Appendix D). Future surveys will have to understand SN lensing, host-galaxy correlations, intrinsic color, evolution, and other effects that have the potential to bias cosmological constraints. Large samples that allow a complicated parameter space to be explored will thus be necessary. For all these reasons, photometric-classification as an underpinning of SN cosmology is here to stay.

We thank the referee, whose diligent but friendly review greatly improved the quality of this paper. We thank Mark Sullivan for many helpful discussions during the lifetime of this project and analysis. We thank Julien Guy for sending us the data plotted in Figure 24, and Alex Conley for his advice with the simple_cosfitter. We thank Michael WoodVasey and Raffaele Flaminio for helpful discussions on earlier drafts of the paper. Additionally, we thank Alex Kim and Rick Kessler for their useful inputs on advanced draft of the paper. We thank Marisa March for her comments on grid versus MCMC search methods. We thank Emma Beynon for her advice with ComsoMC, Rob Crittenden, and David Bacon for useful discussions on statistics. Numerical computations were done on the Sciama High Performance Computer (HPC) cluster, which is supported by the ICG, SEPNet and the University of Portsmouth. We thank Gary Burton for his help in using Sciama HPC.

Funding for the SDSS and SDSS-II has been provided by the Alfred P. Sloan Foundation, the Participating Institutions, the National Science Foundation, the U.S. Department of Energy, the National Aeronautics and Space Administration, the Japanese Monbukagakusho, the Max Planck Society, and the Higher Education Funding Council for England. The SDSS Web site is http://www.sdss.org/. The SDSS is managed by the Astrophysical Research Consortium for the Participating Institutions. The Participating Institutions are the American Museum of Natural History, Astrophysical Institute Potsdam, University of Basel, Cambridge University, Case Western Reserve University, University of Chicago, Drexel University, Fermilab, the Institute for Advanced Study, the Japan Participation Group, Johns Hopkins University, the Joint Institute for Nuclear Astrophysics, the Kavli Institute for Particle Astrophysics and Cosmology, the Korean Scientist Group, the Chinese Academy of Sciences (LAMOST), Los Alamos National Laboratory, the Max-PlanckInstitute for Astronomy (MPIA), the Max-Planck-Institute for Astrophysics (MPA), New Mexico State University, Ohio State University, University of Pittsburgh, University of Portsmouth, Princeton University, the United States Naval Observatory, and the University of Washington.

Funding for SDSS-III has been provided by the Alfred P. Sloan Foundation, the Participating Institutions, the National Science Foundation, and the U.S. Department of Energy. The SDSS-III Web site is http://www.sdss3.org/. SDSS-III is managed by the Astrophysical Research Consortium for the Participating Institutions of the SDSS-III Collaboration including the University of Arizona, the Brazilian Participation Group, Brookhaven National Laboratory, University of Cambridge, University of Florida, the French Participation Group, the German Participation Group, the Instituto de Astrofisica de Canarias, the Michigan State/Notre Dame/JINA Participation Group, Johns Hopkins University, Lawrence Berkeley National Laboratory, Max Planck Institute for Astrophysics, New Mexico State University, New York University, Ohio State University, Pennsylvania State University, University of Portsmouth, Princeton University, the Spanish Participation Group, University of Tokyo, University of Utah, Vanderbilt University, University of Virginia, University of Washington, and Yale University.

\section{APPENDIX A}

\section{PHOTOMETRIC SUPERNOVA IDENTIFICATION}

In this paper we make use of the PSNID (S11) software to obtain a typing of SN candidates using only the photometry of each object. PSNID has been shown to perform better than other photometric-classification algorithms, scoring the highest FoM in the recent classification challenge of K10a. This methodology has been applied to the full three-year data release of the SDSS-II SN Survey and will be presented in full in Sako et al. (in preparation).

We refer the reader to S11 for a full description of PSNID, but describe here some of the key features of the algorithm which are relevant to this paper. PSNID uses the observed multi-color light curve to calculate a reduced $\chi^{2}$ fit to a grid of SN Ia lightcurve models and non-Ia SN templates, assigning probabilities for each SN using the Bayesian evidence criteria. PSNID returns 
the SN Ia Bayesian evidence $\left(E_{\mathrm{Ia}}\right)$, given by,

$$
\begin{aligned}
E_{\mathrm{Ia}}= & \int P\left(z, A_{\mathrm{v}}, T_{\max }, \Delta m_{15, \mathrm{~B}}, \mu\right) e^{-\chi^{2} / 2} \\
& \times d z d A_{\mathrm{v}} d T_{\max } d \Delta m_{15, \mathrm{~B}} d \mu,
\end{aligned}
$$

where the redshift prior $P(z)$ for an externally constrained redshift $z_{\text {ext }}$ is given by

$$
P(z)=\frac{1}{\sqrt{2} \pi \sigma_{\mathrm{z}}} e^{-\left(z-z_{\mathrm{ext}}\right)^{2} / 2 \sigma_{\mathrm{z}}^{2}},
$$

and $\sigma_{\mathrm{z}}^{2}$ is the error on the observed (external) redshift.

The $E_{\mathrm{Ia}}$ is computed by marginalizing the product of the likelihood function and the prior probabilities over the model parameter space. Five parameters are included in the model: redshift $(z)$, host-galaxy extinction $\left(A_{v}\right)$, time of maximum light $\left(T_{\max }\right)$, the amount by which an SN Ia declined in the $B$-band during the first 15 days after maximum light $\left(\Delta m_{15}\right.$; Phillips $1993)$, and the distance modulus $(\mu)$. Flat priors are assumed on $A_{V}, T_{\max }$, and $\Delta m_{15}$. Galactic extinction is corrected for using $A_{\mathrm{V}}$ from Schlegel et al. (1998), assuming the Cardelli et al. (1989) extinction law with $R_{\mathrm{V}}=A_{\mathrm{V}} / E(B-V)=3.1$. The color law for SN host galaxies, however, is assumed to be steeper, with $R_{\mathrm{V}}=2.2$ (Kessler et al. 2009a).

The non-Ia SNe Bayesian evidences $\left(E_{\mathrm{Ibc}, \mathrm{II}}\right)$ are given by

$$
E_{\mathrm{Ibc}, \mathrm{II}}=\sum_{\text {templates }} \int P(z) e^{-\chi^{2} / 2} d z d A_{\mathrm{v}} d T_{\max } d \mu
$$

This quantity is the summation over a variety of Type Ibc and Type II templates, as given in Table 1 of S11. PSNID returns a Bayesian probability for each of the three SN types considered herein, given by

$$
P_{\mathrm{type}}=\frac{E_{\mathrm{type}}}{E_{\mathrm{Ia}}+E_{\mathrm{Ibc}}+E_{\mathrm{II}}},
$$

where, by definition, $P_{\mathrm{Ia}}+P_{\mathrm{Ibc}}+P_{\mathrm{II}}=1$.

We use in this paper a version of PSNID that has incorporated several improvements over that presented in S11. The primary difference is in the light-curve template uncertainties (Equations (6) and (7) from S11); these have been changed to better match the observed distribution of errors in spectroscopically confirmed SDSS-II SNe. Specifically, the magnitude errors $\delta m$ on the SNe Ia gri model light curves are now

$$
\delta m_{\mathrm{Ia}}= \begin{cases}0.06+0.04 \times(|t| / 20) & |t|<20 \text { days } \\ 0.10+0.18 \times((|t|-20) / 60) & |t| \geqslant 20 \text { days }\end{cases}
$$

while the new magnitude errors for the non-Ia SN light-curve templates are

$$
\delta m_{\mathrm{CC}}=0.06+0.08 \times(|t| / 60),
$$

where $t$ is the rest-frame epoch in days from $B$-band maximum. These changes require us to re-determine goodness-of-fit thresholds used in S11 on PSNID, which we describe in Section 3.1.4.

In Section 2.2.1 we obtain a preliminary classification of our SDSS-II SN candidates by running PSNID on the ugriz light curves while placing a flat prior on the redshift. This classification shapes our subsequent target list for BOSS hostgalaxy spectroscopy, and is the only occasion in this paper where we use a flat redshift prior. For both our simulated SNe (Section 3.1.4) and our SN candidates that have host-galaxy spectra from BOSS we use the spectroscopic redshift as our prior (Section 3.2). This additional prior on the redshift helps compress the parameter space being investigated and break degeneracies between non-Ia SNe at low- $z$, which could appear similar to higher redshift SNe Ia. As in S11, we assume flat priors for all other PSNID model parameters.

\section{APPENDIX B}

\section{SPECTRAL ADAPTIVE LIGHT-CURVE TEMPLATE}

We determine the distance moduli for our SN candidates (both data and simulated) using the SALT2 light-curve fitting software (version 2.2; Guy et al. 2010). For each SN light-curve the SALT2 fitting program determines the best-fit value of three parameters $\left(X_{0}, X_{1}, c\right)$, which describe the observed luminosity offset, stretch, and color of the SN, respectively (Guy et al. 2007, 2010). These fitted values are then used to "standardize" the light curve, as the distance modulus to each $\mathrm{SN}$ is calculated using

$$
\mu=m_{* \mathrm{~B}}-M+\alpha x_{1}-\beta c,
$$

where $m_{* \mathrm{~B}}$ is the $B$-band peak apparent magnitude and is defined as $-2.5 \log _{10}\left(x_{0}\right)$. Parameters $\alpha, \beta$, and $M$ (absolute $B$-band magnitude at peak) are constants that can either be derived for the whole sample simultaneously with the best-fit cosmology, or can be constrained from other data. In our cosmology analysis presented in Section 6 we allow $\alpha$ and $\beta$ to float within priors and analytically marginalize over $M$ (which is degenerate with $H_{0}$ ).

We fit (in flux) SALT2 to the SDSS SMP light-curve data in the griz passbands. Although the SALT2 template does not extend to the rest-frame $z$-band, we include $z$-band data in our fits as it should help constrain the model at higher redshifts. However, the $z$ band has low throughput in the SDSS, and we obtain consistent light-curve fits with and without including this information. We exclude $u$-band photometry due to its low $\mathrm{S} / \mathrm{N}$, but we note that this does not significantly affect the quality of our fits, as these data are of much lower quality than in griz.

In Figure 27, we present the SDSS-II light curves for the four SN candidates whose BOSS host-galaxy spectra are shown in Figure 3. We also present the SALT2 best-fit SN Ia model and the $1 \sigma$ error on this fit provided by SALT2.

\section{APPENDIX C}

\section{REDSHIFT COMPARISON}

In Figure 28 we compare the BOSS host-galaxy redshift and the SN spectroscopic redshift for the 186 spectroscopically classified SNe Ia that pass our selection cuts. In only four cases do these redshift measurements disagree significantly (SN3199; SN13956; SN15301; SN19757). Based on visual inspection of the SDSS-III DR8 catalog we identify and remove SN3199 from our sample, as the BOSS-targeted galaxy does not appear to be the most likely SN host galaxy. The remaining three $\mathrm{SNe}$ are retained in our sample as the identified host galaxy appears to be correct and they reside close to the Hubble diagram (small HRs) when using the BOSS host-galaxy redshifts rather than the $\mathrm{SN}$-spectrum redshift. Apart from this check, the purpose of which is to determine the likelihood of host misidentification, we make no other use of SN spectroscopy in this paper.

We have also compared our BOSS host-galaxy redshifts with SDSS galaxy redshifts, where the latter are available. We found 

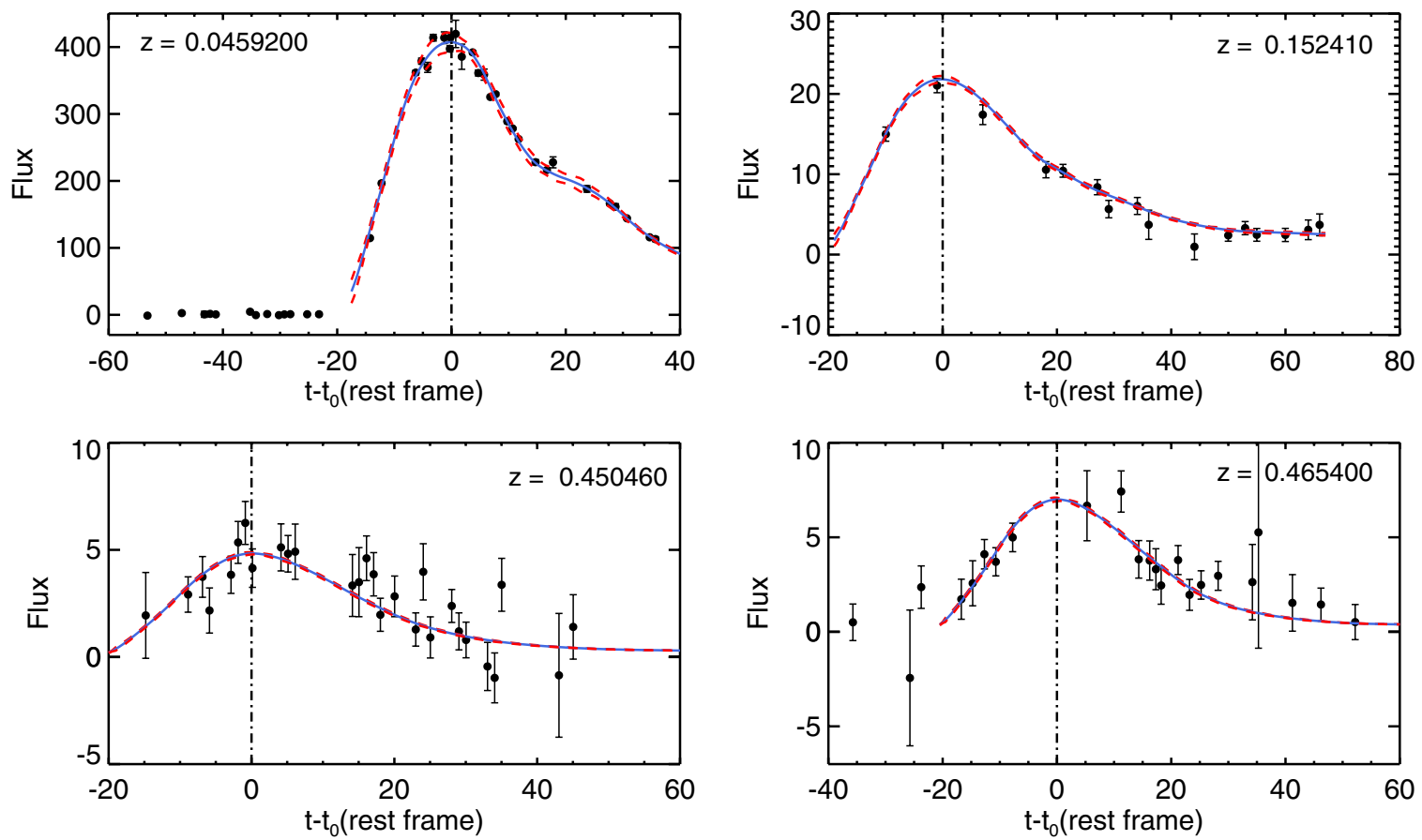

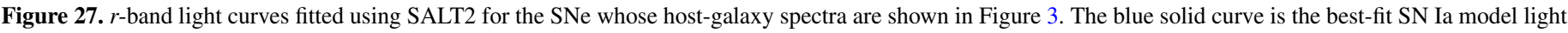
curve, and the red dashed lines represent the $1 \sigma$ uncertainties on this fit. The vertical dotted line shows the best-fit time $t_{0}$ of peak brightness.

(A color version of this figure is available in the online journal.)

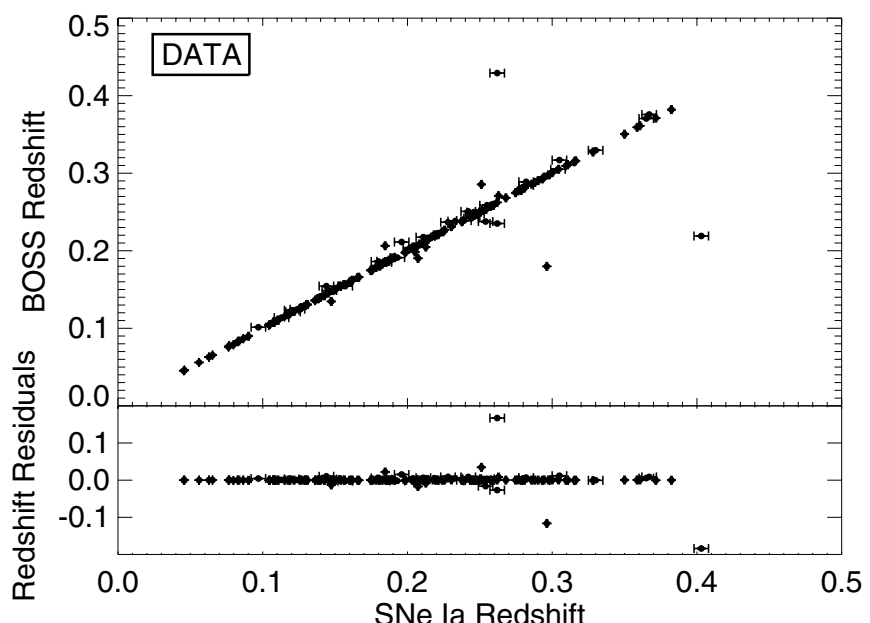

Figure 28. Comparison of the spectroscopic redshift for our spectroscopically confirmed SNe Ia and the corresponding host-galaxy redshift from BOSS. The bottom panel shows the redshift residuals (BOSS galaxy redshift-SNe redshift) for this sample.

one SN Ia (SN6491) hosted in a galaxy where the redshifts from these two surveys disagree, and removed this object from our sample.

Additionally, we have visually inspected the host galaxies of other photometrically classified SNe Ia candidates in our sample. First, we inspected a random subset of 70 photometric SNe Ia, finding no obvious misidentification of the appropriate host. This is reassuring, as it confirms that the rate of misidentification of hosts must be low. Next, we inspected the host association for SNe Ia candidates that are clearly offset from the Hubble diagram in Figure 16 to ensure the correct galaxy had been assigned during targeting. We found only one host galaxy that was likely to be incorrect (SN9052), being located 25" from our photometrically classified SN, and removed this object from our sample. In total we removed only two $\mathrm{SNe}$ Ia from our sample because of likely host-galaxy mismatches.

\section{APPENDIX D \\ OTHER SYSTEMATIC UNCERTAINTIES}

There are a number of other systematic uncertainties that are likely to affect our SDSS-II SN sample beyond the photometricclassification specific uncertainties discussed in Section 7.1. As outlined in Kessler et al. (2009a) and Conley et al. (2011), present SN samples have major uncertainties associated with their photometric calibration and the light-curve fitting technique used, as well as many astrophysical uncertainties such as correlations with host-galaxy properties, SN lensing, peculiar velocities, galactic dust, and SN evolution. Though we do not address these important systematics in detail in this paper, we discuss the likely effect of these additional systematic uncertainties on our results.

The optimal method of light-curve fitting is not known; there can be significant differences in the cosmological results obtained from using different algorithms (e.g., SALT2 and MLCS2k2; Kessler et al. 2009a; Sollerman et al. 2009). Both Guy et al. (2010) and Conley et al. (2011) find consistent cosmological results between SALT2 and a different technique (SiFTO), with a possible systematic uncertainty of only $\Delta \mu \simeq$ $0.02-0.03$ mag between light-curve fitters. We have chosen to use only the SALT2 light-curve fitting algorithm (Guy et al. 2010) in this work.

As discussed in Conley et al. (2011) for SNLS, the most important systematic uncertainty in present $\mathrm{SN}$ surveys is the photometric calibration. Therefore, Conley et al. (2011, p. 45) recommended that future $\mathrm{SN}$ surveys should be calibrated onto a "more modern, better understood photometric systems such as USNO/SDSS." By using photometric data obtained wholly 
Table 6

Table Containing Data for 10 of the 752 Photometrically Classified SNe Ia Presented in This Paper

\begin{tabular}{|c|c|c|c|c|c|c|c|c|c|c|c|c|c|}
\hline \multirow[t]{2}{*}{ CID } & \multirow[t]{2}{*}{$z$} & \multirow{2}{*}{$\begin{array}{c}z \text { err } \\
\left(10^{-5}\right)\end{array}$} & \multicolumn{2}{|c|}{$\mathrm{SNe} \mathrm{Ia}$} & \multicolumn{2}{|c|}{ Host Galaxy } & \multirow{2}{*}{$\begin{array}{c}\text { Host ObsID } \\
\text { (DR8) }\end{array}$} & \multicolumn{3}{|c|}{ SALT2 } & \multicolumn{3}{|c|}{$\mu$} \\
\hline & & & R.A. & Decl. & R.A. & Decl. & & $X_{0}\left(10^{-5}\right)$ & $X_{1}$ & Color & Uncorrected & Corrected & Err \\
\hline 703 & 0.2980 & 0.63 & -23.7821 & 0.6507 & -23.7821 & 0.6507 & 1237663544222483004 & 1.34 & 0.63 & -0.01 & 40.73 & 40.77 & 0.18 \\
\hline 762 & 0.1914 & 0.75 & 15.5354 & -0.8790 & 15.5354 & -0.8790 & 1237666338114765068 & 3.08 & 1.15 & -0.01 & 39.93 & 39.94 & 0.15 \\
\hline 779 & 0.2381 & 0.66 & 26.6737 & -1.0206 & 26.6737 & -1.0206 & 1237657069548208337 & 1.90 & 0.38 & 0.01 & 40.22 & 40.25 & 0.16 \\
\hline 822 & 0.2376 & 4.46 & 40.5608 & -0.8622 & 40.5608 & -0.8622 & 1237657584950379049 & 1.66 & -0.52 & -0.07 & 40.44 & 40.46 & 0.18 \\
\hline 859 & 0.2783 & 0.60 & -9.4483 & 0.3866 & -9.4483 & 0.3866 & 1237666408438301119 & 1.61 & 0.56 & 0.02 & 40.42 & 40.45 & 0.17 \\
\hline 893 & 0.1101 & 0.81 & 5.4942 & -0.1317 & 5.4942 & -0.1317 & 1237657190907641944 & 1.87 & -1.05 & 0.11 & 39.67 & 39.68 & 0.16 \\
\hline 1112 & 0.2576 & 0.83 & -20.9825 & -0.3752 & -20.9825 & -0.3752 & 1237663478724428434 & 1.37 & -0.41 & -0.03 & 40.55 & 40.57 & 0.19 \\
\hline 1119 & 0.2978 & 0.36 & -39.5865 & 0.8946 & -39.5865 & 0.8946 & 1237663458851619714 & 1.30 & 0.55 & -0.14 & 41.12 & 41.16 & 0.25 \\
\hline 1166 & 0.3821 & 3.10 & 9.3556 & 0.9733 & 9.3556 & 0.9733 & 1237663716555293384 & 0.85 & 1.36 & 0.00 & 41.33 & 41.41 & 0.23 \\
\hline 1241 & 0.0898 & 0.48 & -22.3274 & -0.7766 & -22.3274 & -0.7766 & 1237656567586226517 & 12.88 & -0.55 & 0.04 & 37.87 & 37.87 & 0.14 \\
\hline
\end{tabular}

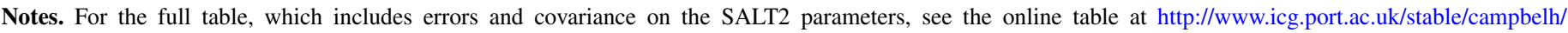

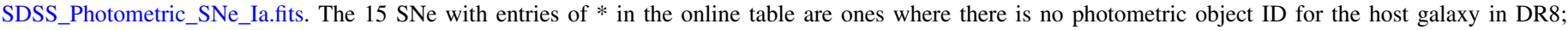
these galaxies do appear in the co-added images, and from this catalog we quote the HostID.

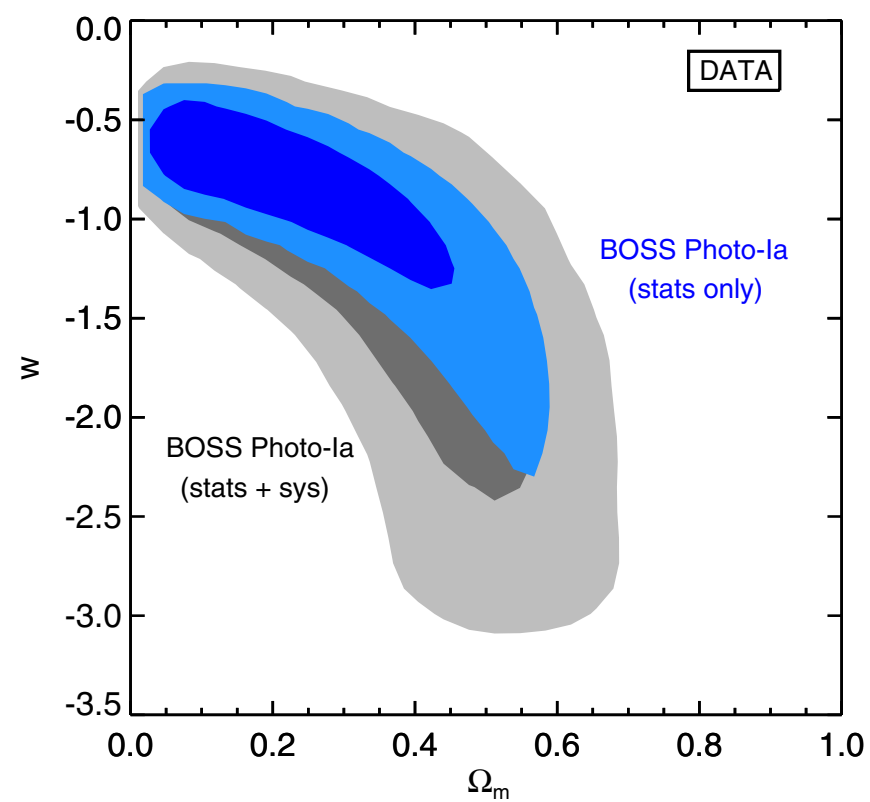

Figure 29. $68 \%$ and $95 \%$ confidence contours of $w$ and $\Omega_{\mathrm{m}}$ in a flat $w \mathrm{CDM}$ model. We assume a Gaussian prior on $H_{0}$; other priors are summarized in Table 5, using prior Set III. The blue contours show the statistics only contours and the gray contours include the estimate of the systematics, as well as statistical uncertainties.

(A color version of this figure is available in the online journal.)

from the SDSS-II SN Survey, we believe we have minimized calibration uncertainties, as the SDSS photometric system is now mature and well understood (Ivezić et al. 2007; Doi et al. 2010). Mosher et al. (2012) have recently compared the SDSS-II photometric system to that of the CSP using light-curve data for nine $\mathrm{SNe}$ Ia observed concurrently by the two projects. They conclude that measurements from the two surveys agree in all bands at or better than $2 \%$ in flux, and are consistent with no difference in $g$ - and $r$-band magnitude at the $2 \sigma$ level. This is an indication of the relative calibration between the SDSS and other surveys (CSP in this case) and not a direct statement on the absolute calibration, which would require observation of a known source like NIST photodiodes, as discussed by Stubbs \& Tonry (2012). Such techniques will be implemented in future surveys (e.g., DES). Based on these findings, we assume systematic uncertainty of only $\Delta \mu \approx 0.02$ mag on the distance modulus of our $\mathrm{SNe}$, relative to other surveys.

The next largest systematic uncertainty at present is the recently discovered correlation between the (corrected) peak absolute magnitude of SNe Ia and the stellar mass of the host galaxy (Kelly et al. 2010; Sullivan et al. 2010; Lampeitl et al. 2010b). This relationship has been observed to have an effect as large as $\Delta \mu \simeq 0.07 \mathrm{mag}$ in the SDSS-II SN sample (Lampeitl et al. 2010b). We do not attempt to make this correction here, as it is beyond the scope of this paper, but we note it is being investigated in M. Smith et al. (in preparation). There will likely be significant stellar population modeling uncertainties associated with determining reliable stellar masses of our faint host galaxies; the large magnitude errors on the photometric colors of our BOSS galaxies make this especially difficult at $z>0.3$. We will revisit the topic of SN Ia correlations with hostgalaxy properties using our photometrically classified sample in a future paper.

There are a number of other astrophysical uncertainties that could be considered, especially SN lensing effects, peculiar velocities, and possible SN evolution. These are all smaller in size compared to the systematic uncertainties discussed above, and should be further mitigated in our sample because of the lack of relatively high-redshift SNe Ia where these effects are most prominent (especially lensing and evolution).

In Figure 29 we show an estimate of the effect of systematic uncertainties on our results, compared to Figure 25 in Section 6.3 which includes only statistical errors. These measurements are obtained by adding in quadrature an additional uncertainty of $\Delta \mu=0.1 \mathrm{mag}$ to the distance moduli errors of our observed SNe Ia; the same methodology as Kowalski et al. (2008). This level of uncertainty is an estimate from the combination (in quadrature) of uncertainties associated with the light-curve fitter (0.03 mag), photometric calibration (0.02 mag), lensing and peculiar velocities $(0.05 \mathrm{mag})$, and possible host-galaxy correlations $(0.07 \mathrm{mag})$. When including our systematic errors in the cosmological analysis we fix the SALT2 SN parameters $\alpha$ and $\beta$ to the values found in Section 6; this is done to prevent $\alpha$ and $\beta$ changing values to counteract the increase in dispersion that the systematic errors cause. The results do not change significantly with our inclusion of these estimates of the unknown systematics, giving a best fit of $w=-0.93_{-0.49}^{+0.39}$ and $\Omega_{m}=0.314_{-0.06}^{+0.07}$. However, we stress again that this analysis is not comprehensive. 


\section{APPENDIX E}

\section{PHOTOMETRICALLY CLASSIFIED SNe Ia DATA}

In Table 6 we present the key information used in this paper for our sample of 752 photometrically classified SNe Ia. The full SDSS-II SN sample, including all the light-curve data, redshifts, and classifications for all transients, will be published in Sako et al. (in preparation). Table 6 includes a unique identification number for the whole SDSS-II SN Survey (CID; Column 1), BOSS host-galaxy redshift and error (Columns 2 and 3), the R.A. and decl. (in degrees) of the SN event (Columns 4 and 5) and its host (Columns 6 and 7), a unique SDSS object identifier for the host galaxy from DR8 (Column 8), the SALT2 parameters $X_{0}$ in flux units, $X_{1}$ and color both in magnitudes (Columns 9, 10, 11 ), and finally the uncorrected and corrected distance modulus (Columns 12 and 13) with error (Column 14), all in magnitudes.

The data in Table 6, along with the SALT2 covariance matrices and the SN type probabilities, can be electronically downloaded from http://www.icg.port.ac.uk/ campbelh. The probabilities listed there are those used in this paper (with the BOSS host-galaxy redshift prior) and include the probability assigned to each object of being an $\mathrm{SNe}$ Ia $\left(P_{\mathrm{Ia}}\right)$, a Type II SN $\left(P_{\mathrm{II}}\right)$, and a Type $\mathrm{Ibc}\left(P_{\mathrm{Ibc}}\right)$.

\section{REFERENCES}

Abazajian, K. N., Adelman-McCarthy, J. K., Agüeros, M. A., et al. 2009, ApJS, 182,543

Ahn, C. P., Alexandroff, R., Allende-Prieto, C., et al. 2012, ApJS, 203, 21 Akaike, H. 1974, ITAC, 19, 716

Aldering, G., Adam, G., Antilogus, P., et al. 2002, Proc. SPIE, 4836, 61 Amanullah, R., Lidman, C., Rubin, D., et al. 2010, ApJ, 716, 712 Anderson, L., Aubourg, E., Bailey, S., et al. 2012, MNRAS, 427, 3435 Bailey, S., Aldering, G., Antilogus, P., et al. 2009, A\&A, 500, L17 Barris, B. J., \& Tonry, J. L. 2004, ApJL, 613, 21

Bazin, G., Palanque-Delabrouille, N., Rich, J., et al. 2009, A\&A, 499, 653

Bazin, G., Ruhlmann-Kleider, V., Palanque-Delabrouille, N., et al. 2011, A\&A, 534, A43

Bernstein, J. P., Kessler, R., Kuhlmann, S., et al. 2012, ApJ, 753, 25

Blanton, M. R., \& Roweis, S. 2007, AJ, 133, 734

Blondin, S., Matheson, T., Kirshner, R. P., et al. 2012, AJ, 143, 126

Bloom, J. S., Kasen, D., Shen, K. J., et al. 2012, ApJL, 744, 17

Bolton, A. S., Schlegel, D. J., Aubourg, E., et al. 2012, AJ, 144, 144

Bridle, S. L., Crittenden, R., Melchiorri, A., et al. 2002, MNRAS, 335, 1193

Bronder, T. J., Hook, I. M., Astier, P., et al. 2007, A\&A, 477, 717

Brooks, S., \& Gelman, A. 1998, J. Comput. Graph. Stat., 7, 434

Cardelli, J. A., Clayton, G. C., \& Mathis, J. S. 1989, ApJ, 345, 245

Chotard, N., Gangler, E., Aldering, G., et al. 2011, A\&A, 529, L4

Clarkson, C., Ellis, G., Faltenbacher, A., et al. 2012, MNRAS, 426, 1121

Conley, A., Guy, J., Sullivan, M., et al. 2011, ApJS, 192, 1

Conley, A., Sullivan, M., Hsiao, E. Y., et al. 2008, ApJ, 681, 482

Contreras, C., Hamuy, M., Phillips, M. M., et al. 2009, BAAS, 41, 696

D’Andrea, C. B., Gupta, R. R., Sako, M., et al. 2011, ApJ, 743, 172

Dawson, K. S., Schlegel, D. J., Ahn, C. P., et al. 2013, AJ, 145, 10

Dilday, B., Bassett, B., Becker, A., et al. 2010, ApJ, 715, 1021

Dilday, B., Howell, D. A., Cenko, S. B., et al. 2012, arXiv:1001.4995

Dilday, B., Kessler, R., Frieman, J. A., et al. 2008, ApJ, 682, 262

Doi, M., Tanaka, M., Fukugita, M., et al. 2010, AJ, 139, 1628

Drake, A. J., Djorgovski, S. G., Mahabal, A., et al. 2009, ApJ, 696, 870

Eisenstein, D. J., Weinberg, D. H., Agol, E., et al. 2011, AJ, 142, 72

Ellis, R. S., Sullivan, M., Nugent, P. E., et al. 2008, ApJ, 674, 51

Falck, B. L., Riess, A. G., \& Hlozek, R. 2010, ApJ, 723, 398

Filippenko, A. V. 1997, ARA\&A, 35, 309

Folatelli, G., Phillips, M. M., Burns, C. R., et al. 2010, AJ, 139, 120

Foley, R. J., Filippenko, A. V., \& Jha, S. W. 2008, ApJ, 686, 117

Foley, R. J., Filippenko, A. V., Kessler, R., et al. 2010a, ApJ, 748, 127

Foley, R. J., Filippenko, A. V., Kessler, R., et al. 2012, AJ, 143, 113

Foley, R. J., Matheson, T., Blondin, S., et al. 2009, AJ, 137, 3731

Foley, R. J., Narayan, G., Challis, P. J., et al. 2010b, ApJ, 708, 1748

Frieman, J. A., Bassett, B., Becker, A., et al. 2008, AJ, 135, 338

Fukugita, M., Ichikawa, T., Gunn, J. E., et al. 1996, AJ, 111, 1748
Galbany, L., Miquel, R., Ostman, L., et al. 2012, ApJ, 755, 125

Gallagher, J. S., Garnavich, P. M., Berlind, P., et al. 2005, ApJ, 634, 210

Gallagher, J. S., Garnavich, P. M., Caldwell, N., et al. 2008, ApJ, 685, 752

Ganeshalingam, M., Li, W., Filippenko, A. V., et al. 2010, ApJS, 190, 418

Gjergo, E., Duggan, J., Cunningham, J. D., et al. 2012, JCAP, submitted (arXiv:1205.1480)

Gong, Y., Cooray, A., \& Chen, X. 2010, ApJ, 709, 1420

Gunn, J., Carr, M., Rockosi, C., \& Sekiguchi, M. 1998, AJ, 116, 3040

Gunn, J. E., Siegmund, W. A., Mannery, E. J., et al. 2006, AJ, 131, 2332

Gupta, R. R., D’Andrea, C. B., Sako, M., et al. 2011, ApJ, 740, 92

Guy, J., Astier, P., Baumont, S., et al. 2007, A\&A, 466, 11

Guy, J., Sullivan, M., Conley, A., et al. 2010, A\&A, 523, A7

Hamuy, M., Folatelli, G., Morrell, N. I., et al. 2006, PASP, 118, 2

Hamuy, M., Phillips, M., Suntzeff, N. B., Schommer, R. A., \& Maza, J. 1996a, AJ, 112, 2408

Hamuy, M., Phillips, M. M., Suntzeff, N. B., et al. 1996b, AJ, 112, 2391

Hicken, M., Wood-Vasey, W. M., Blondin, S., et al. 2009a, ApJ, 700, 1097

Hicken, M., Wood-Vasey, W. M., Blondin, S., et al. 2009b, ApJ, 700, 1097

Hlozek, R., Kunz, M., Bassett, B., et al. 2012, ApJ, 752, 19

Holtzman, J. A., Marriner, J., Kessler, R., et al. 2008, AJ, 136, 2306

Howell, D. A., \& Legacy Survey, S. 2009, BAAS, 41, 484

Howell, D. A., Sullivan, M., Nugent, P. E., et al. 2006, Natur, 443, 308

Hoyle, F., \& Fowler, W. A. 1960, ApJ, 132, 565

Iben, I., Jr., \& Tutukov, A. V. 1984, ApJS, 54, 335

Ishida, E. E. O., \& de Souza, R. S. 2012, arXiv:1201.6676

Ivezić, Ž., Smith, J. A., Miknaitis, G., et al. 2007, AJ, 134, 973

Jarosik, N., Bennett, C. L., Dunkley, J., et al. 2011, ApJS, 192, 14

Jha, S., Kirshner, R. P., Challis, P., Garnavich, P. M., \& Matheson, T. 2006, AJ, 131,527

Jha, S., Riess, A. G., \& Kirshner, R. P. 2007, ApJ, 659, 122

Johnson, B. D., \& Crotts, A. P. S. 2006, AJ, 132, 756

Kelly, P. L., Hicken, M., Burke, D. L., Mandel, K. S., \& Kirshner, R. P. 2010, ApJ, 715,743

Kessler, R., Bassett, B., Belov, P., et al. 2010a, PASP, 122, 1415

Kessler, R., Becker, A. C., Cinabro, D., et al. 2009a, ApJS, 185, 32

Kessler, R., Bernstein, J. P., Cinabro, D., et al. 2009b, PASP, 121, 1028

Kessler, R., Conley, A., Jha, S., \& Kuhlmann, S. 2010b, arXiv:1001.5210

Kessler, R., Guy, J., Marriner, J., et al. 2012, ApJ, submitted (arXiv:1209.2482)

Knights, M., Bassett, B. A., Varughese, M., et al. 2012, arXiv:1205.3493

Knop, R. A., Aldering, G., Amanullah, R., et al. 2003, ApJ, 598, 102

Konishi, K., Yasuda, N., Tokita, K., et al. 2011, AJ, submitted (arXiv:1101.1565)

Kowalski, M., Rubin, D., Aldering, G., et al. 2008, ApJ, 686, 749

Kunz, M., Bassett, B. A., \& Hlozek, R. 2007, PhRvD, 75, 103508

Kuznetsova, N. V., \& Connolly, B. M. 2007, ApJ, 659, 530

Lampeitl, H., Nichol, R. C., Seo, H.-J., et al. 2010a, MNRAS, 401, 2331

Lampeitl, H., Smith, M., Nichol, R. C., et al. 2010b, ApJ, 722, 566

Larson, D., Dunkley, J., Hinshaw, G., et al. 2011, ApJS, 192, 16

Law, N. M., Kulkarni, S. R., Dekany, R. G., et al. 2009, PASP, 121, 1395

Leloudas, G., Chatzopoulos, E., Dilday, B., et al. 2012, A\&A, 541, A129

Lewis, A., \& Bridle, S. 2002, PhRvD, 66, 103511

Lidman, C., Ruhlmann-Kleider, V., Sullivan, M., et al. 2012, arXiv:1205.1306

Li, W., Armstrong, M., Boles, T., Itagaki, K., \& Nakano, S. 2003, IAUC, 8245,1

Li, W., Bloom, J. S., Podsiadlowski, P., et al. 2011, Natur, 480, 348

LSST Science Collaboration, Abell, P. A., Allison, J., et al. 2009 , arXiv:0912.0201

Lupton, R., Gunn, J., \& Szalay, A. 1999, AJ, 118, 1406

Maguire, K., Sullivan, M., Thomas, R. C., et al. 2011, MNRAS, 418, 747

Malmquist, K. G. 1936, Medd. Stockholmes Obs., 26

Mannucci, F. 2005, in ASP Conf. Ser. 342, 1604-2004: Supernovae as Cosmological Lighthouses, ed. M. Turatto, S. Benetti, L. Zampieri, \& W. Shea (San Francisco, CA: ASP), 140

Marriner, J., Bernstein, J. P., Kessler, R., et al. 2011, ApJ, 740, 72

McClelland, C. M., Garnavich, P. M., Galbany, L., et al. 2010, ApJ, 720, 704

Miknaitis, G., Pignata, G., Rest, A., et al. 2007, ApJ, 666, 674

Mosher, J., Sako, M., Corlies, L., et al. 2012, AJ, 144, 17

Newling, J., Bassett, B. A., Hlozek, R., et al. 2011, arXiv:1110.6178

Nomoto, K. 1982, ApJ, 253, 798

Nordin, J., Östman, L., Goobar, A., et al. 2011, ApJ, 734, 42

Nugent, P. E., Sullivan, M., Cenko, S. B., et al. 2011, arXiv:1110.6201

Ostman, L., Nordin, J., Goobar, A., et al. 2012, A\&A, 526, A28

Perlmutter, S., Aldering, G., Goldhaber, G., et al. 1999, ApJ, 517, 565

Phillips, M. M. 1993, ApJL, 413, 105

Phillips, M. M., Li, W., Frieman, J. A., et al. 2007, PASP, 119, 360

Pier, J. R., Munn, J. A., Hindsley, R. B., et al. 2003, AJ, 125, 1559

Poznanski, D., Gal-Yam, A., Maoz, D., et al. 2002, PASP, 114, 833

Poznanski, D., Maoz, D., \& Gal-Yam, A. 2007, AJ, 134, 1285 
Pskovskii, I. P. 1977, SvA, 21, 675

Quimby, R. M., Kulkarni, S. R., Kasliwal, M. M., et al. 2011, Natur, 474, 487

Reid, B. A., Percival, W. J., Eisenstein, D. J., et al. 2010, MNRAS, 404, 60

Richards, J. W., Homrighausen, D., Freeman, P. E., Schafer, C. M., \& Poznanski, D. 2012, MNRAS, 419, 1121

Riess, A. G., Filippenko, A. V., Challis, P., et al. 1998, AJ, 116, 1009

Riess, A. G., Kirshner, R. P., Schmidt, B. P., et al. 1999, AJ, 117, 707

Riess, A. G., Macri, L., Casertano, S., et al. 2009, ApJ, 699, 539

Riess, A. G., Macri, L., Casertano, S., et al. 2011, ApJ, 730, 119

Riess, A. G., Press, W. H., \& Kirshner, R. P. 1996, ApJ, 473, 88

Riess, A. G., Strolger, L.-G., Casertano, S., et al. 2007, ApJ, 659, 98

Riess, A. G., Strolger, L.-G., Tonry, J., et al. 2004, ApJ, 607, 665

Rodney, S. A., \& Tonry, J. L. 2009, ApJ, 707, 1064

Rodney, S. A., \& Tonry, J. L. 2010, ApJ, 715, 323

Sako, M., Bassett, B., Becker, A., et al. 2008, AJ, 135, 348

Sako, M., Bassett, B., Connolly, B., et al. 2011, ApJ, 738, 162

Scalzo, R. A., Akerlof, C., Aldering, G., et al. 2010, BAAS, 42, 45

Schlegel, D. J., Finkbeiner, D. P., \& Davis, M. 1998, ApJ, 500, 525

Scolnic, D. M., Riess, A. G., Huber, M. E., et al. 2009, ApJ, 706, 94
Silverman, J. M., Ganeshalingam, M., Li, W., \& Filippenko, A. V. 2012, MNRAS, 425, 1889

Smartt, S. J. 2009, ARA\&A, 47, 63

Smee, S., Gunn, J. E., Uomoto, A., et al. 2012, AJ, submitted (arXiv:1208.2233)

Smith, M., Nichol, R. C., Dilday, B., et al. 2012, ApJ, 755, 24

Sollerman, J., Mörtsell, E., Davis, T. M., et al. 2009, ApJ, 703, 1374

Stritzinger, M., Phillips, M. M., S., L. B., et al. 2011, AJ, 142, 156

Stubbs, C. W., \& Tonry, J. L. 2012, in Proc. Conf. Calibration and Standardization of Large Surveys and Missions in Astron. and Astrophys., submitted (arXiv:1206.6695)

Sullivan, M., Conley, A., Howell, D. A., et al. 2010, MNRAS, 406, 782

Sullivan, M., Guy, J., Conley, A., et al. 2011, ApJ, 737, 102

Sullivan, M., Howell, D. A., Perrett, K., et al. 2006, AJ, 131, 960

Suzuki, N., Rubin, D., Lidman, C., et al. 2012, ApJ, 746, 85

Tonry, J. L., Schmidt, B. P., Barris, B., et al. 2003, ApJ, 594, 1

Tripp, R. 1997, A\&A, 325, 871

Walker, E. S., Hook, I. M., Sullivan, M., et al. 2010, MNRAS, 410, 1262

Wang, L., Hoeflich, P., \& Wheeler, J. C. 1997, ApJL, 483, 29

Wood-Vasey, W. M., Miknaitis, G., Stubbs, C. W., et al. 2007, ApJ, 666, 694

York, D. G., Adelman, J., Anderson, J. E., Jr., et al. 2000, AJ, 120, 1579

Zheng, C., \& Romani, R. 2008, AJ, 135, 1766 